\title{
POWER-ASSOCIATIVE RINGS
}

\author{
BY \\ A. A. ALBERT \\ INTRODUCTION
}

We use the term ring for any additive abelian group closed with respect to a product operation such that the two-sided distributive law holds. When the associative law for products also holds we call the ring an associative ring. Every element $x$ of any ring $\mathfrak{A}$ generates a subring $\mathfrak{A}(x)$ of $\mathfrak{A}$ consisting of all finite sums of terms each of which is a finite product whose factors are all equal to $x$. We call $\mathfrak{A}$ a power-associative ring when every $\mathfrak{A}(x)$ is an associative subring of $\mathfrak{A}$.

We have shown elsewhere $\left({ }^{1}\right)$ that a ring $\mathfrak{A}$ whose characteristic is zero is power-associative if and only if $x x^{2}=x^{2} x$ and $x^{2} x^{2}=\left(x^{2} x\right) x$ for every $x$ of $\mathfrak{A}$. This result is also true for all commutative rings having characteristic prime to 30 , and the stated restrictions on the characteristic are actually necessary.

Our present investigation begins with a derivation of results on the decompositions of a power-associative ring relative to its idempotents. When $e$ is an idempotent of a commutative power-associative ring $\mathfrak{A}$, the corresponding (right) multiplication $R_{e}$ is an endomorphism of $A$ having simple elementary divisors and roots $0,1 / 2,1$. There is a resulting decomposition of $\mathfrak{A}$ as the supplementary sum $\mathfrak{A}_{e}(1)+\mathfrak{A}_{e}(1 / 2)+\mathfrak{A}_{e}(0)$ of submodules $\mathfrak{A}_{e}(\lambda)$ such that $x e=\lambda x$. Moreover the multiplication relations for these submodules are nearly those holding for the case $\left({ }^{2}\right)$ where $\mathfrak{A}$ is a Jordan ring. However, the situation becomes much more complicated when $\mathfrak{A}$ is not commutative since then the elementary divisors of $R_{e}$ need not be simple and the characteristic roots are quite arbitrary.

It is true, nevertheless, that a decomposition theory may be obtained for all power-associative rings $\mathfrak{A}$ in which the equation $2 x=a$ has a unique solution $x$ in $\mathfrak{A}$ for every $a$ of $\mathfrak{A}$. In this case we may always attach to $\mathfrak{A}$ a commutative ring $\mathfrak{A}^{(+)}$which is the same additive group as $\mathfrak{A}$ and which has a product $x \cdot y$ defined in terms of the product $x y$ of $\mathfrak{A}$ by $2(x \cdot y)=x y+y x$. The ring $\mathfrak{A}^{(+)}$is power-associative when $\mathfrak{A}$ is, and every idempotent of $\mathfrak{A}$ is also an idempotent of $\mathfrak{A}^{(+)}$. This yields a decomposition of $\mathfrak{A}=\mathfrak{A}_{e}(1)+\mathfrak{A}_{e}(1 / 2)$ $+\mathfrak{A}_{e}(0)$ where $\mathfrak{A}_{e}(\lambda)$ is the set of all $x$ such that $x e+e x=2 \lambda x$, and the submodules always have some of the multiplicative properties of the Jordan

Presented to the Society, September 5, 1947, and November 29, 1947; received by the editors October 25, 1947. 21-33.

(1) On the power-associativity of rings, Summa Braziliensis Mathematicae vol. 2 (1948) pp.

(2) Cf. $\$ 10$ of the author's $A$ structure theory for Jordan algebras, Annals of Mathematics vol. 48 (1947) pp. 546-567. 
case. The list of properties grows when we assume that $\mathfrak{A}$ is a flexible ring, that is, $x(y x)=(x y) x$ for every $x$ and $y$ of $\mathfrak{A}$. It becomes essentially complete when $\mathfrak{A}^{(+)}$is assumed to have one further property of Jordan rings.

While the theory of the decomposition relative to an idempotent is a basic part of a general structure theory for power-associative rings and algebras, one can hardly hope to derive a complete structure theory even for commutative power-associative algebras. It then becomes desirable to restrict the study by a proper selection of additional hypotheses. One possible line of investigation lies in the study of what are known as shrinkable algebras. We shall discuss all commutative shrinkable algebras of shrinkability level two here, and shall show that those algebras belonging to the classes of algebras containing algebras with a unity element are either Jordan algebras or are defined by the identity $x^{2} y^{2}+(x y)^{2}=\left(x^{2} y\right) y+\left(y^{2} x\right) x$. We shall also give a structure theory for algebras of the latter type.

A second line of investigation consists of an attempt at generalizing the Jordan algebra so as to delete the commutative law. We shall give a two postulate definition of a class of algebras including both Jordan and associative algebras and shall give a complete structure theory for these "standard" algebras. The simple standard algebras turn out to be merely associative or Jordan algebras and so this investigation does not yield any new types of simple algebras.

The final line of investigation we shall present here is a complete determination of those algebras $\mathfrak{A}$ such that $\mathfrak{A}^{(+)}$is a simple Jordan algebra. We are first led to attach to any algebra $\mathfrak{B}$ over a field $\mathfrak{F}$ an algebra $\mathfrak{B}(\lambda)$ defined for every $\lambda$ of $\mathfrak{F}$. This algebra is the same vector space over $\mathfrak{F}$ as $\mathfrak{B}$ but the product $x \cdot y$ in $\mathfrak{B}(\lambda)$ is defined in terms of the product $x y$ of $\mathfrak{B}$ by $x \cdot y=\lambda x y$ $+(1-\lambda) y x$. We then call an algebra $\mathfrak{A}$ over $\mathfrak{F}$ a quasiassociative algebra if there exists a scalar extension $\Re$ of $\mathfrak{F}$ (necessarily of degree $n=1,2$ over $\mathfrak{F}$ ), a quantity $\lambda$ in $\Re$, and an associative algebra $\mathfrak{B}$ over $\Re$, such that $\mathfrak{A}_{\Re}=\mathfrak{B}(\lambda)$. The structure of quasiassociative algebras is readily determined and we shall conclude our work by showing that if $\mathfrak{A}^{(+)}$is a simple Jordan algebra then $\mathfrak{A}$ is either $\mathfrak{U}^{(+)}$or is a simple quasiassociative algebra.

\section{Chapter I. Nilrings AND idempotents}

1. Power-associativity. While our results on power-associativity have been published elsewhere $\left({ }^{2}\right)$ they may not be accessible readily and so will be summarized here.

If $\mathfrak{A}$ is any ring we shall say that the characteristic of $\mathfrak{A}$ is prime to $n$ if the sum $n x=0$ only if $x=0$. We shall also say that $\mathfrak{A}$ has characteristic zero providing that the characteristic is prime to $n$ for all integers $n>1$.

The right powers of the elements $x$ of a ring $\mathfrak{A}$ are defined by the formula $x^{k+1}=x^{k} x, k \geqq 1$. Then $\mathfrak{A}$ is power-associative if and only if $x^{\alpha} x^{\beta}=x^{\alpha+\beta}$ for all positive integers $\alpha, \beta$. Assume first that the characteristic of $\mathfrak{A}$ is prime to 
two and define

$$
[x, y]=x y-y x \text {. }
$$

Then the hypothesis $x^{3}=x x^{2}$ may be written as $\left[x, x^{2}\right]=0$ and a linearization process implies that

$$
[x y+y x, z]+[y z+z y, x]+[z x+x z, y]=0 .
$$

Conversely (1) implies that $x x^{2}=x^{2} x$ if the characteristic of $\mathfrak{A}$ is prime to six. Formula (1) may then be used to obtain

Lemma 1. Let the characteristic of $\mathfrak{A}$ be prime to two, $n \geqq 4$, and $x^{\lambda} x^{\mu}=x^{\lambda+\mu}$ for all positive integers $\lambda, \mu$ such that $\lambda+\mu<n$. Then

$$
n\left[x^{n-1}, x\right]=0, \quad\left[x^{n-\alpha}, x^{\alpha}\right]=\alpha\left[x^{n-1}, x\right] \quad(\alpha=1, \cdots, n-1) .
$$

The hypothesis $x^{2} x^{2}=x^{3} x$ yields, as the consequence of a linearization process, the relation

$$
\sum_{6}(x y+y x)(z w+w z)=\sum_{4}\left[\sum_{3}(z w+w z) y\right] x
$$

for any ring $\mathfrak{A}$ whose characteristic is prime to two. Here the sums are taken over all possible selections of the symbols involved and $\sum_{k}$ is a sum of $k$ terms. Conversely (3) implies that $x^{2} x^{2}=x^{3} x$ providing that the characteristic of $\mathfrak{A}$ is prime to six.

If the characteristic of $\mathfrak{A}$ is prime to two, the symbol $2^{-1}\left[x^{n-1}, x\right]$ has meaning, since either $\left[x^{n-1}, x\right]=0$ or $\left[x^{n-1}, x\right] \neq 0, m\left[x^{n-1}, x\right]=0$, and $2^{-1}\left[x^{n-1}, x\right]=\eta\left[x^{n-1}, x\right]$ where $m \xi+2 \eta=1$ and $m$ is an odd divisor of $n$. We use this concept in the statement of

Lemma 2. Let $\mathfrak{A}$ be a ring whose characteristic is prime to $30, n \geqq 5, x^{\lambda} x^{\mu}$ $=x^{\lambda+\mu}$ for $\lambda+\mu<n$. Then

$$
x^{n-\alpha} x^{\alpha}=x^{n-1} x+\frac{\alpha-1}{2}\left[x^{n-1}, x\right] \quad(\alpha=1, \cdots, n-1),
$$

from which we may derive $\left[x^{n-\alpha}, x^{\alpha}\right]=\alpha\left[x^{n-1}, x\right], n\left[x^{n-1}, x\right]=0$ and thus $x^{n-\alpha} x^{\alpha}=x^{n}$ if $n$ is prime to the characteristic of $\mathfrak{A}$.

The proof of the lemma above is made by replacing $x$ by $x^{\alpha}, y$ by $x^{\beta}, z$ by $x^{\gamma}$, $w$ by $x^{\delta}$ in (3) where $\alpha+\beta+\gamma+\delta=n$. It has the following consequences.

LemMA 3. Let $\mathfrak{A}$ be a ring of characteristic zero and $x^{2} x=x x^{2}, x^{2} x^{2}=\left(x^{2} x\right) x$ for every $x$ of $\mathfrak{A}$. Then $\mathfrak{A}$ is power-associative.

LEMma 4. Let $\mathfrak{A}$ be a commutative ring whose characteristic is prime to 30 and let $x^{2} x^{2}=\left(x^{2} x\right) x$. Then $\mathfrak{A}$ is power-associative. 
The conditions on the characteristic given in these results are actually necessary as has been shown $\left(^{1}\right)$ by counterexamples.

2. The ring $\mathfrak{U}^{(+)}$. We shall restrict our attention to power-associative rings whose characteristic is prime to six. We shall also assume that the equation $2 x=a$ has a unique solution $x$ in $\mathfrak{A}$ for every $a$ of $\mathfrak{A}$. Then we may define an attached ring $\mathfrak{A}^{(+)}$which is the same additive group as $\mathfrak{A}$ but which has a product operation $x \cdot y$ defined in terms of the product operation $x y$ of $\mathfrak{A}$ by $2(x \cdot y)=x y+y x$. The ring $\mathfrak{A}^{(+)}$is a commutative ring and powers in $\mathfrak{Y}^{(+)}$coincide with powers in $\mathfrak{A}$. Thus $\mathfrak{U}^{(+)}$is power-associative. Note that the construction of $\mathfrak{A}^{(+)}$could yield a power-associative ring even when $\mathfrak{A}$ itself is not power-associative. Indeed consider the algebra $\mathfrak{A}$ over the field $\mathfrak{F}$ with a basis $a, a^{2}, a a^{2}, a^{2} a$ defined so that all products $a \cdots a$ with $n \geqq 4$ factors are zero. In $\mathfrak{A}^{(+)}$the identity $x \cdot x^{2}=x^{2} \cdot x$ is a trivial consequence of the commutative law, and $x^{\alpha} \cdot x^{\beta}=0$ if $\alpha+\beta \geqq 4, x^{\alpha} \cdot x^{\beta}=x^{\alpha+\beta}$ for all positive integers $\alpha, \beta$. Then $\mathfrak{A}^{(+)}$is power-associative but $a a^{2} \neq a^{2} a$ in $\mathfrak{A}$.

In any ring $\mathfrak{A}$ the mapping $a \rightarrow a x$ is an endomorphism $R_{x}$ of the additive group $\mathfrak{A}$ and is called a right multiplication of $\mathfrak{A}$. Similarly the endomorphism $L_{x}$ defined by $a \rightarrow x a=a L_{x}$ is called a left multiplication of $\mathfrak{A}$. Under the assumption we have made, every endomorphism $S$ of $\mathfrak{A}$ determines a unique endomorphism $S / 2$ and the mapping

$$
T_{x}=2^{-1}\left(R_{x}+L_{x}\right)
$$

is the unique endomorphism of $\mathfrak{A}$ defining the generic multiplication $a \cdot x$ $=a T_{x}$ of $\mathfrak{A}^{(+)}$. We shall use this property when we apply the theory of the decomposition of a commutative ring relative to an idempotent to noncommutative rings.

3. Multiplication identities. If $\mathfrak{A}$ is a power-associative ring whose characteristic is prime to six we have in (1) a result which may be written as

$$
R_{x y+y x}-L_{x y+y x}=\left(R_{x}+L_{x}\right)\left(R_{y}-L_{y}\right)+\left(R_{y}+L_{y}\right)\left(R_{x}-L_{x}\right) .
$$

Also (3) holds and may be written as

$$
\begin{aligned}
L_{(x y+y x) z}+L_{(y z+z y) x} & +L_{(z x+x z) y} \\
= & \left(R_{x}+L_{x}\right)\left(R_{y z+z y}+L_{y z+z y}-R_{y} R_{z}-R_{z} R_{y}\right) \\
& +\left(R_{y}+L_{y}\right)\left(R_{x z+z x}+L_{x z+z x}-R_{x} R_{z}-R_{z} R_{x}\right) \\
& +\left(R_{z}+L_{z}\right)\left(R_{x y+y x}+L_{x y+y x}-R_{x} R_{y}-R_{y} R_{x}\right) \\
& -\left(L_{x y+y x} R_{z}+L_{x z+z x} R_{y}+L_{y z+z y} R_{x}\right) .
\end{aligned}
$$

Take $x=y$ in (6) and obtain

$$
R_{x x}-L_{x x}=\left(R_{x}+L_{x}\right)\left(R_{x}-L_{x}\right) .
$$

Also take $y=z=x$ in (7) and obtain 


$$
L_{(x x) x}=\left(R_{x}+L_{x}\right)\left(R_{x x}+L_{x x}-R_{x}^{2}\right)-L_{x x} R_{x} .
$$

We may now prove

LEMMA 5. Let $\mathfrak{A}_{x}$ be the set of all finite sums of products of finite numbers of factors equal to $R_{x}, L_{x}$ or $R_{x x}$ so that $\mathfrak{A}_{x}$ is either zero or an associative ring for every $x$ of $\mathfrak{A}$. Then $\mathfrak{A}_{x}$ contains $R_{w}$ and $L_{w}$ for all positive integral powers $w=x^{k}$ of $x$.

The result has already been shown true for $k=1,2$ by (8). Assume it true for $k \leqq t+1$ and write $w=x^{t+2}$. Put $y=x^{t+1}$ in (6) and have, since $x y+y x=2 w$,

$$
2\left(R_{w}-L_{w}\right)=\left(R_{x}+L_{x}\right)\left(R_{y}-L_{y}\right)+\left(R_{y}+L_{y}\right)\left(R_{x}-L_{x}\right) .
$$

Next replace $z$ by $x^{t}$ and $y$ by $x$ in (7) so that $(x y+y x) z=(y z+z y) x=(z x$ $+x z) y=6 w$. The right member of $(7)$ is then in $\mathfrak{A}_{x}$ by the hypothesis of our induction, $L_{w}$ is in $\mathfrak{A}_{x}, R_{w}$ is in $\mathfrak{A}_{x}$ by (10).

In the particular case where $x=e=e^{2}$ formulas (8) and (9) take the form

$$
\begin{gathered}
\left(R_{e}+L_{e}-I\right)\left(R_{e}-L_{e}\right)=0, \\
\left(R_{e}+L_{e}\right)^{2}-\left(R_{e}+L_{e}\right) R_{e}^{2}-L_{e}\left(R_{e}+I\right)=0,
\end{gathered}
$$

where $I$ is the identity endomorphism of $\mathfrak{A}$. It is not possible to eliminate $R_{\bullet}$ or $L_{\theta}$ between these two equations so as to obtain a fixed characteristic equation of finite degree for either $R_{e}$ or $L_{\boldsymbol{e}}$. For the characteristic roots of $R_{\boldsymbol{e}}$, in the case of a noncommutative power-associative algebra, may be completely arbitrary. We shall give an example illustrating this property later.

When $\mathfrak{A}$ is commutative (7) becomes

$$
\begin{aligned}
& R_{x(y z)}+R_{y(z x)}+R_{z(x y)} \\
& =4\left(R_{x} R_{y z}+R_{y} R_{z x}+R_{z} R_{x y}\right)-\left(R_{y z} R_{x}+R_{z x} R_{y}+R_{x y} R_{z}\right) \\
& \quad-\left[R_{x}\left(R_{y} R_{z}+R_{z} R_{y}\right)+R_{y}\left(R_{z} R_{x}+R_{x} R_{z}\right)+R_{z}\left(R_{x} R_{y}+R_{y} R_{x}\right)\right],
\end{aligned}
$$

and (6) is vacuous. Also (8) is vacuous while (9) becomes

$$
R_{(x x) x}=4 R_{x} R_{x x}-R_{x x} R_{x}-2 R_{x}^{3} \text {. }
$$

We may then use (13) with $y=x, z=x^{2}$ to obtain a relation which may be combined with (14) to yield

$$
R_{(x x)(x x)}=R_{x x}^{2}+10 R_{x}^{2} R_{x x}-6 R_{x} R_{x x} R_{x}-4 R_{x}^{4} .
$$

We shall use these formulas later.

4. Nilrings and nilideals. In a power-associative ring $\mathfrak{A}$ all positive integral powers $a^{k}$ of any element $a$ of $\mathfrak{A}$ are uniquely determined and so the meaning of the nilpotency of the elements of $\mathfrak{A}$ should be clear. We shall say that $\mathfrak{A}$ is a nilring if all elements of $\mathfrak{A}$ are nilpotent and shall say that $\mathfrak{A}$ has 
bounded index $t>1$ if $a^{t}=0$ for every $a$ of $\mathfrak{A}, b^{t-1} \neq 0$ for some element $b$ of $\mathfrak{A}$.

An ideal $\mathfrak{B}$ of $\mathfrak{A}$ will be called a nilideal if $\mathfrak{B} \neq 0$ is a nilring. The sum of two nilideals is a nilideal. For if $\mathfrak{B}$ and $\mathfrak{C}$ are nilideals their sum $(\mathfrak{B}, \mathfrak{E})$ consists of all sums $b+c$ of elements $b$ of $\mathfrak{B}$ and $c$ of $\mathfrak{C}$. Then $b^{k}=0,(b+c)^{k}$ $=b^{k}+c_{1}=c_{1}$ is in $\mathfrak{( E , ~} c_{1}^{t}=(b+c)^{k t}=0$ for some $t$. Thus $(\mathfrak{B}$, () is a nilideal.

It follows trivially that the sum $\left(\mathfrak{B}_{1}, \cdots, \mathfrak{B}_{r}\right)$ of any finite number of nilideals is a nilideal. We define the union $\mathfrak{N}$ of all nilideals of $\mathfrak{A}$ to be the set of all finite sums $b=b_{1}+\cdots+b_{r}$ where $b_{i}$ is in a nilideal $\mathfrak{B}_{i}$ of $\mathfrak{A}$. Then $b$ is in $\left(\mathfrak{B}_{1}, \cdots, \mathfrak{B}_{r}\right)$ and is nilpotent. Evidently $\mathfrak{R}$ is a nilideal of $\mathfrak{A}$. We shall call $\mathfrak{N}$ the nilradical of $\mathfrak{A}$ and see that $\mathfrak{A}-\mathfrak{N}$ must have no nilideals.

In the case of a Jordan algebra $\mathfrak{A}$ the maximal nilideal of $\mathfrak{A}$ actually coincides with the maximal solvable ideal of $\mathfrak{A}$ and is, indeed, the maximal nilpotent ideal of $\mathfrak{A}$ in the sense that there exists an integer $k$ such that all products of $k$ elements of $\mathfrak{N}$ are zero. In the general power-associative ring case no such result is to be expected and indeed every simple Lie algebra is a nilring. One can then hardly expect to be able to prove that a nilring is nilpotent but a limited result of this type is provable.

THEOREM 1. Let $\mathfrak{A}$ be a commutative power-associative ring of nilindex $t \leqq 4$. Then $\mathfrak{A}_{x}$ is zero or a nilpotent associative ring for every $x$ of $\mathfrak{A}$.

We first observe that if $\mathfrak{A}$ has nilindex two then $(a+b)^{2}=a^{2}+2 a b+b^{2}$ $=2 a b=0$ and so $a b=0, \mathfrak{A}$ is a zero ring, $\mathfrak{A}_{x}$ consists of the zero endomorphism only. We next let $t=3$ so that there exists an element $z$ in $\mathfrak{A}$ such that $z^{2} \neq 0$, $R_{z} \neq 0$. The relation $x^{2} x=0$ implies that

$$
(x y) z+(x z) y+(y z) x=0 .
$$

Indeed the left member of (16) arises in the derivation of (1) from the term $x^{2} x$ in $x^{2} x=x x^{2}$. But then

$$
R_{x y}+R_{x} R_{y}+R_{y} R_{x}=0 .
$$

Then $R_{x x}=-2 R_{x}^{2}, R_{x x}$ is commutative with $R_{x}$ and $\mathfrak{A}$ is actually a Jordan ring. Also $R_{(x x) x}=-2 R_{x} R_{x x}=4 R_{x}^{3}=0$ by (17) and so $\mathfrak{A}_{x}$ is actually generated by the nilpotent endomorphism $R_{x}, \mathfrak{A}_{x}$ is nilpotent of nilindex at most three. In the case where $\mathfrak{A}$ is a Jordan algebra it is actually known that the union of all of the algebras $\mathfrak{A}_{x}$ is a nilpotent algebra.

There remains the case $t=4$. The hypothesis $x^{2} x^{2}=\left(x^{2} x\right) x$ implies that

$$
\begin{aligned}
4[(x y)(z w) & +(x z)(y w)+(x w)(y z)] \\
= & x[y(z w)+z(w y)+w(y z)]+y[x(z w)+z(w x)+w(x z)] \\
& +z[x(y w)+y(w x)+w(x y)]+w[x(y z)+y(z x)+z(x y)]
\end{aligned}
$$

when $\mathfrak{A}$ is commutative, and the left member of (18) arises from the linearization of $x^{2} x^{2}$. Then $x^{2} x^{2}=0$ implies that the left member of (18) is zero, that is, 


$$
R_{x} R_{y z}+R_{y} R_{z x}+R_{z} R_{x y}=0 .
$$

It follows that $R_{x} R_{x x}=0$ and we may use (15) to obtain $R_{x x}^{2}=4 R_{x}^{4}$. But then $R_{x} R_{x x}^{2}=4 R_{x}^{5}=0, R_{x}$ is nilpotent. To prove that $\mathfrak{A}_{x}$ is nilpotent we observe that the quantities of $\mathfrak{A}_{x}$ are finite sums $S$ of products $S_{1} \cdots S_{\alpha}$ where $\alpha \geqq 1$ and $S_{i}=R_{x}$ or $S_{i}=R_{x x}$. Then every product of six elements of $\mathfrak{A}_{x}$ is a finite sum of products $P=S_{1} \cdots S_{\beta}$ where $\beta \geqq 6$. If $S_{1}=R_{x}$ then $P=0$ regardless of the values of the $S_{j}$. If $S_{1}=R_{x x}=S_{2}$ then $S_{1} S_{2}=4 R_{x}^{4}$ and $S_{1} S_{2} S_{3}=4 R_{x}^{5}$ or $4 R_{x}^{4} R_{x x}$ and in either case $P=0$. Finally let $S_{1}=R_{x x}$ and $S_{2}=R_{x}$. Then $S_{2} \cdots S_{6}=0$ in every case and $P=0, \mathfrak{A}_{x}$ is nilpotent.

We shall leave open the question as to whether or not commutative nilrings of index $t \geqq 5$ possess the property of the theorem and pass on now to the case of a noncommutative ring not possessing this property. Indeed, let $\mathfrak{A}$ be an algebra over a field $\mathfrak{F}$ with a basis $u, v$, uv over $\mathfrak{F}$ such that

$$
\begin{gathered}
u^{2}=v^{2}=(u v)^{2}=0, \quad v u+u v=0, \\
(u v) u=-u(u v)=v, \quad(u v) v=-v(u v)=u .
\end{gathered}
$$

Then if $x=\lambda u+\mu v+\nu u v$ the quantity $x^{2}=\lambda^{2} u^{2}+\mu^{2} v^{2}+\nu^{2}(u v)^{2}+\lambda \mu(u v+v u)$ $+\lambda \nu[u(u v)+(u v) u]+\mu \nu[v(u v)+(u v) v]=0$. It follows that $\mathfrak{A}$ is a powerassociative nilring of nilindex two. But evidently both of the transformations $R_{u}$ and $R_{v}$ are not nilpotent. Indeed, their matrices are

$$
\left(\begin{array}{rrr}
0 & 0 & 0 \\
0 & 0 & -1 \\
0 & 1 & 0
\end{array}\right), \quad\left(\begin{array}{lll}
0 & 0 & 1 \\
0 & 0 & 0 \\
1 & 0 & 0
\end{array}\right),
$$

and $R_{u}^{4}$ and $R_{v}^{2}$ are idempotent.

It is easily verified that the algebra defined above is a simple Lie algebra. Indeed, if $\mathfrak{A}$ is any simple Lie algebra then $\mathfrak{A}$ has nilindex two. However Engel's theorem states that the multiplications $R_{x}$ are all nilpotent only when $\mathfrak{A}$ is nilpotent. Note that even in the case of the solvable Lie algebra with a basis $u, v$, $u v$ such that $u^{2}=v^{2}=(u v)^{2}=(u v) v=v(u v)=0, v u=-u v,(u v) u$ $=-u(u v)=v$ we have $R_{u}^{4}$ idempotent.

5. Idempotents in a commutative ring. In a commutative power-associative ring (12) becomes

$$
2 R_{e}^{3}-3 R_{e}^{2}+R_{e}=\left(2 R_{e}-I\right)\left(R_{e}-I\right) R_{e}=0
$$

for every idempotent $e$ of $\mathfrak{A}$. Define $\mathfrak{A}_{e}(\lambda)$ to be the set of all elements $a_{e}(\lambda)$ of $\mathfrak{A}$ such that

$$
e a_{e}(\lambda)=\lambda a_{e}(\lambda),
$$

where (20) implies that necessarily $\lambda=0,1,1 / 2$. Then $\mathfrak{A}_{\theta}(\lambda)$ is a submodule of $\mathfrak{A}$ and it is actually true that $\mathfrak{A}$ is the supplementary sum 


$$
\mathfrak{A}=\mathfrak{A}_{e}(1)+\mathfrak{A}_{e}(1 / 2)+\mathfrak{A}_{e}(0) .
$$

In fact every $a$ of $\mathfrak{A}$ is uniquely expressible in the form $a_{e}(1)+a_{\theta}(1 / 2)+a_{e}(0)$ where

$$
\begin{aligned}
a_{e}(1) & =a\left(2 R_{e}^{2}-R_{e}\right), \\
a_{e}(1 / 2) & =4 a\left(R_{e}-R_{e}^{2}\right), \\
a_{e}(0) & =a\left(I-3 R_{e}+2 R_{e}^{2}\right) .
\end{aligned}
$$

That $a_{e}(\lambda)$ is in $\mathfrak{A}_{e}(\lambda)$ follows by forming the product $e a_{e}(\lambda)$ and using (20). The uniqueness is then a consequence of the fact that if $a_{e}(1)+a_{e}(1 / 2)$ $+a_{e}(0)=0$ then we may multiply by $e$ to obtain $a_{e}(1)+2^{-1} a_{e}(1 / 2)=0, a_{e}(1)$ $+4^{-1} a_{e}(1 / 2)=0,4^{-1} a_{e}(1 / 2)=a_{e}(1 / 2)=0=a_{e}(1)=a_{e}(0)$. We now prove

TheOREM 2. The modules $\mathfrak{H}_{e}(1)$ and $\mathfrak{R}_{e}(0)$ in a commutative power-associative ring are zero or orthogonal subrings of $\mathfrak{A}$. They are related to $\mathfrak{A}_{e}(1 / 2)$ by the inclusion relations

$$
\begin{aligned}
\mathfrak{A}_{e}(1 / 2) \mathfrak{A}_{e}(1 / 2) & \leqq \mathfrak{A}_{\ell}(1)+\mathfrak{A}_{\Theta}(0), \\
\mathfrak{A}_{e}(1) \mathfrak{A}_{e}(1 / 2) & \leqq \mathfrak{A}_{e}(1 / 2)+\mathfrak{A}_{e}(0), \\
\mathfrak{A}_{e}(0) \mathfrak{A}_{e}(1 / 2) & \leqq \mathfrak{A}_{e}(1 / 2)+\mathfrak{A}_{e}(1)
\end{aligned}
$$

To prove these relations we put $z=w=e$ in (18) and let $x e=\lambda x, y e=\mu y$. This yields

$$
\begin{aligned}
& 2[(x y) e](\lambda+\mu)+2[(x y) e] e+(x y)\left[2 \lambda^{2}+\lambda+2 \mu^{2}+\mu\right] \\
&=8 \lambda \mu x y+4(x y) e .
\end{aligned}
$$

When $\lambda=\mu=1$ we obtain $(x y)\left(R_{e}^{2}-I\right)=0$ whence $(x y)\left(R_{e}-I\right)=0$, that is, $x y$ is in $\mathfrak{A}_{\theta}(1), \mathfrak{A}_{\theta}(1)$ is a subring of $\mathfrak{A}$. Similarly $\lambda=\mu=0$ yields $(x y)\left(R_{e}^{2}-2 R_{e}\right)$ $=(x y) R_{e}\left(R_{e}-2 I\right)=0, R_{e}-2 I$ is nonsingular, $(x y) R_{e}=(x y) e=0$ and $\mathfrak{A}_{e}(0)$ is a subring of $\mathfrak{A}$. The values $\lambda=1, \mu=0$ yield $(x y)\left(2 R_{e}^{2}-2 R_{e}+3 I\right)=0$ and so $x y\left(2 R_{e}^{3}-2 R_{e}^{2}+3 R_{e}\right)=0$. By $(20), x y\left(R_{e}^{2}+2 R_{e}\right)=0, x y R_{e}=0,3 x y=0, x y=0$. There remains the case of products $\mathfrak{A}_{\theta}(\lambda) \mathfrak{A}_{\theta}(1 / 2)$, that is, $\mu=1 / 2$. If $\lambda=1$ then $2 \lambda^{2}+\lambda+2 \mu^{2}+\mu-8 \lambda \mu=2+1+1-4=0$ and $(x y)\left(2 R_{e}^{2}-R_{e}\right)=0, \quad x y$ is in $\mathfrak{A}_{e}(1 / 2)+\mathfrak{A}_{e}(0)$ by $(23)$. If $\lambda=0$ then $(x y)\left(2 R_{e}^{2}-3 R_{e}+I\right)=0$ and $x y$ is in $\mathfrak{A}_{e}(1 / 2)+\mathfrak{A}_{e}(1)$. The remaining value $\lambda=1 / 2$ yields $(x y)\left(R_{e}^{2}-R_{e}\right)=0$, that is, $x y$ is in $\mathfrak{A}_{e}(1)+\mathfrak{A}_{e}(0)$.

In the case of Jordan algebras the last two inclusion relations of (24) may be replaced by the sharper result stating that

$$
\mathfrak{A}_{e}(1) \mathfrak{A}_{e}(1 / 2) \leqq \mathfrak{A}_{e}(1 / 2), \quad \mathfrak{A}_{e}(0) \mathfrak{A}_{e}(1 / 2) \leqq \mathfrak{A}_{e}(1 / 2)
$$

However (26) does not hold for all commutative power-associative rings. Indeed, let $\mathfrak{A}$ be an algebra over a field $\mathfrak{F}$ of characteristic not 2,3 , or 5 and let 
$\mathfrak{U}$ have a basis $e, f, g, h$ with a multiplication table given by $e^{2}=e, e f=f$, $e g=2^{-1} g, g h=f, e h=f^{2}=g^{2}=h^{2}=f g=f h=0$ and the commutative law. Then $\mathfrak{A}_{e}(1)=e \mathfrak{F}+f \mathfrak{F}, \mathfrak{A}_{e}(1 / 2)=g \mathfrak{F}, \mathfrak{A}_{e}(0)=h \mathfrak{F}, \mathfrak{A}_{e}(0) \mathfrak{A}_{e}(1 / 2)=f \mathfrak{F} \leqq \mathfrak{A}_{e}(1), f \mathfrak{F}$ is not in $\mathfrak{A}_{e}(1 / 2)$ and $(20)$ does not hold. Moreover $\mathfrak{A}$ is power-associative since if $x=\alpha e+\beta f+\gamma g+\delta h$ for $\alpha, \beta, \gamma, \delta$ in $\mathfrak{F}$ then $x^{2}=\alpha^{2} e+2(\alpha \beta+\gamma \delta) f+\alpha \gamma g$, $x^{2} x^{2}=\alpha^{4} e+4 \alpha^{2}(\alpha \beta+\gamma \delta) f+\alpha^{3} \gamma g, \quad x^{2} x=\alpha^{3} e+3 \alpha(\alpha \beta+\gamma \delta) f+\alpha^{2} \gamma g, \quad\left(x^{2} x\right) x=\alpha^{4} e$ $+3 \alpha^{2}(\alpha \beta+\gamma \delta) f+\alpha^{3} \gamma g+\alpha^{3} \beta f+\alpha^{2} \gamma \delta f=x^{2} x^{2}$.

6. Idempotents in a noncommutative ring. An idempotent of a powerassociative ring $\mathfrak{A}$ is also an idempotent of $\mathfrak{U}^{(+)}$. Since the additive group $\mathfrak{U}^{(+)}$is precisely the same additive group as $\mathfrak{A}$ we may write (22) where the quantities $a_{e}(\lambda)$ of $\mathfrak{A}_{e}(\lambda)$ are now defined by

$$
e a_{e}(\lambda)+a_{e}(\lambda) e=2 \lambda a_{e}(\lambda) \quad(\lambda=0,1,1 / 2) .
$$

We may then prove

Theorem 3. The submodules $\mathfrak{A}_{\odot}(1)$ and $\mathfrak{A}_{e}(0)$ are orthogonal and such that

$$
\begin{aligned}
& e a_{e}(1)=a_{e}(1) e=a_{e}(1), \\
& e a_{e}(0)=a_{e}(0) e=0
\end{aligned}
$$

for all elements $a_{e}(1)$ of $\mathfrak{A}_{e}(1), a_{e}(0)$ of $\mathfrak{A}_{e}(0)$.

To prove this result we use the relation (1) with $y=z=e$ and $x e+e x=\nu x$ to obtain $2 x e+2 \nu e x=2 e x+2 \nu x e,(1-\nu) x e=(1-\nu) e x$, so that $x e=e x$ when $\nu \neq 1$. Then $\nu=2 \lambda, 2 e x=2 \lambda x$ and $e x=x e=\lambda x$ for $\lambda=0,1$. This yields (28). We

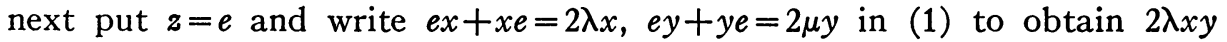
$+2 \mu y^{\prime} x+(x y+y x) e=2 \lambda y x+2 \mu x y+e(x y+y x)$. If $\lambda=1$ and $\mu=0$ then $x y+y x$ $=0$ by Theorem 2 and so $2 x y=2 y x, x y=y x, 2 x y=0, x y=y x=0$ as desired.

The characteristic roots of $R_{e}$ need not be limited to the values $0,1 / 2,1$ of the commutative case if $\mathfrak{A}$ is not a commutative ring. Indeed, consider the algebra $\mathfrak{A}$ with a basis $e, e_{1}, \cdots, e_{t}$ such that $e e_{i}=\alpha_{i} e_{i}, e_{i} e=\left(1-\alpha_{i}\right) e_{i}, e_{i} e_{j}=0$ for $i, j=1, \cdots, t$. Then the characteristic roots $\alpha_{i}$ are arbitrary and we need only prove that $\mathfrak{A}$ is power-associative. We note that if $y=\xi_{1} e_{1}+\cdots+\xi_{t} e_{t}$ then $y^{2}=0$ and so $y y^{2}=y^{2} y, y^{2} y^{2}=\left(y^{2} y\right) y$. For all other quantities $x$ of $\mathfrak{A}$ we have $x=\lambda e+y$ where $\lambda \neq 0$ and the homogeneity of our relations implies that we may take $x=e+y$. But $e y+y e=y$ so that $x^{2}=e+y=x$ and the properties $x x^{2}=x^{2} x, x^{2} x^{2}=\left(x^{2} x\right) x$ are trivial.

It is also not true that the elementary divisors of $R_{e}$ are simple. For consider an algebra with a basis $e, f, g$ such that $g=e f, e^{2}=e, f^{2}=g^{2}=f g=g f$ $=e g=0, f e=f-g, g e=g$. The matrix of $R_{e}$ is

$$
\left(\begin{array}{rrr}
1 & 0 & 0 \\
0 & 1 & -1 \\
0 & 0 & 1
\end{array}\right)
$$


and $R_{e}-I \neq 0,\left(R_{e}-I\right)^{2}=0$. To show that $\mathfrak{A}$ is power-associative we note that if $y=\beta f+\gamma g$ then $y^{2}=0$. It then suffices to prove that $x x^{2}=x^{2} x, x^{2} x^{2}$ $=\left(x^{2} x\right) x$ for $x=e+y, y=\beta f+\gamma g, e y+y e=\beta g+\beta(f-g)+\gamma g=y, x^{2}=x$ and our proof is complete.

Let us next observe the algebra with a basis $e, u, v$ over a field $\mathfrak{F}$ of characteristic prime to 30 such that $e^{2}=e, e u=u e=u / 2, e v=v e=v / 2, u v=u$, $v u=-u, u^{2}=v^{2}=0$. In this noncommutative algebra $\mathfrak{A}_{e}(1 / 2)$ is actually a subalgebra of $\mathfrak{A}$ and so the first relation of (24) does not hold. However if $x=\alpha e+\beta u+\gamma v$ then $x^{2}=\alpha^{2} e+\alpha(\beta u+\gamma v)=\alpha x$, so that the relations implying that $\mathfrak{A}$ is power-associative are trivial.

We shall finally give an example showing that the subsets $\mathfrak{A}_{e}(1)$ and $\mathfrak{A}_{e}(0)$ need not be subrings of $\mathfrak{A}$. We verify this result only for $\mathfrak{A}_{e}(0)$ but an example for the remaining case would be easy to construct. Let $\mathfrak{A}$ be an algebra with a basis $e, u, v, w$ where $e u=u e=u, e^{2}=e, e v=v e=e w=w e=u^{2}=v^{2}=w^{2}=u v$ $=v u=u w=w u=0, v w=u=-w v$. Then $\mathfrak{A}_{e}(0) \mathfrak{A}_{e}(0)=u \mathfrak{F} \leqq \mathfrak{A}_{e}(1)$. If $x=\alpha e$ $+\beta u+\gamma v+\delta w$ then $x^{2}=\alpha^{2} e+2 \alpha \beta u$, and so $x^{2} x=\alpha^{3} e+3 \alpha^{2} \beta u=x x^{2},\left(x^{2} x\right) x$ $=\alpha^{4} e+4 \alpha^{3} \beta u=x^{2} x^{2}, \mathfrak{A}$ is power associative.

It should be noted that if $\mathfrak{A}$ is a vector space over a field $\mathfrak{F}$ and $a e=\lambda a$, $e a=\mu a$ for $\lambda$ and $\mu$ in $\mathfrak{F}$ then either $a$ is in $\mathfrak{A}_{e}(1)$, or in $\mathfrak{A}_{e}(0)$ or in $\mathfrak{A}_{e}(1 / 2)$. For $a e+e a=(\lambda+\mu) a=2 a_{e}(1)+a_{\epsilon}(1 / 2)$. But then the uniqueness of (22) implies that $(\lambda+\mu) a_{e}(0)=0,(\lambda+\mu-1) a_{e}(1 / 2)=0,(\lambda+\mu-2) a_{e}(1)=0$, and only one of the quantities $a_{e}(\alpha)$ can be different from zero. When $a$ is in $\mathfrak{A}_{e}(1)$ we have $\lambda=\mu=1$, and when $a$ is in $\mathfrak{A}_{e}(0)$ we have $\lambda=\mu=0$. However when $a$ is in $\mathfrak{A}_{e}(1 / 2)$ we know only that $\lambda+\mu=1$.

The examples we have given demonstrate that the relatively simple properties of a commutative power associative ring do not hold in general for noncommutative rings and so it is desirable to adjoin additional hypotheses.

7. Flexible rings. A ring $\mathfrak{A}$ will be called a flexible ring if $(x y) x=x(y x)$ for every $x$ and $y$ of $\mathfrak{A}$. Then $[(x+z) y](x+z)=(x+z)[y(x+z)]$ and multiplication and the use of the flexible law yields

$$
(x y) z+(z y) x=x(y z)+z(y x),
$$

a relation holding for every $x, y$, and $z$ of $\mathfrak{A}$. Conversely, if $\mathfrak{A}$ satisfies (29) and the characteristic of $\mathfrak{A}$ is prime to two then $\mathfrak{A}$ is flexible.

The property $x^{2} x=x x^{2}$ holds for all flexible rings. Indeed, we may prove

TheOREM 4. Let $\mathfrak{A}$ be a flexible ring whose characteristic is prime to 30 . Then $\mathfrak{A}$ is power associative if and only if $x^{2} x^{2}=\left(x^{2} x\right) x$ for every $x$ of $\mathfrak{A}$.

For proof we assume that $x^{\alpha} x^{\beta}=x^{\alpha+\beta}$ for all $\alpha+\beta<n$, where $n \geqq 4$, and put $y=x^{\lambda-1}, z=x^{n-\lambda}$ in (29) where $\lambda=2, \cdots, n-1$. Then $x^{\lambda} x^{n-\lambda}+x^{n-1} x=x x^{n-1}$ $+x^{n-\lambda} x^{\lambda}$. Take $\lambda=n-1$ and obtain $2 x^{n-1} x=2 x x^{n-1}$ and so $x^{n-1} x=x x^{n-1}$, $x^{n-\lambda} x^{\lambda}=x^{\lambda} x^{n-\lambda}$. We now apply Lemma 2 and see that $x^{\alpha} x^{\beta}=x^{\alpha+\beta}$, for $\alpha+\beta=n$, 
that is, that $\mathfrak{A}$ is power associative.

The flexible law does not seem to yield the first of the relations of (24) for noncommutative rings. However it does yield the remaining relations in their noncommutative form and we shall state the results as

TheOREM 5. Let e be an idempotent of a flexible power associative ring $\mathfrak{A}$. Then $\mathfrak{A}_{e}(1)$ and $\mathfrak{A}_{e}(0)$ are zero or subrings of $\mathfrak{A}$, ex and xe are in $\mathfrak{A}_{e}(1 / 2)$ for every $x$ of $\mathfrak{A}_{e}(1 / 2)$,

$$
\begin{aligned}
& \mathfrak{A}_{\Theta}(1) \mathfrak{A}_{e}(1 / 2) \leqq \mathfrak{A}_{\Theta}(1 / 2)+\mathfrak{A}_{e}(0), \quad \mathfrak{A}_{\Theta}(1 / 2) \mathfrak{A}_{e}(1) \leqq \mathfrak{A}_{\odot}(1 / 2)+\mathfrak{A}_{\odot}(0), \\
& \mathfrak{A}_{e}(0) \mathfrak{A}_{e}(1 / 2) \leqq \mathfrak{A}_{e}(1 / 2)+\mathfrak{A}_{e}(1), \quad \mathfrak{A}_{e}(1 / 2) \mathfrak{A}_{e}(0) \leqq \mathfrak{A}_{e}(1 / 2)+\mathfrak{A}_{e}(1) \text {. }
\end{aligned}
$$

Put $y e=e y=\alpha y$ and take $z=e$ in (29). Then $(x y) e+\alpha y x=\alpha(x y)+e(y x)$. Hence $\alpha(x y+y x)-e(x y+y x)=2 \alpha x y-[(x y) e+e(x y)]$. It follows that $(x y+y x)\left(\alpha I-L_{e}\right)=2 x y\left(\alpha I-T_{e}\right)$, where $T_{e}=\left(R_{e}+L_{e}\right) / 2$ is the multiplication for $e$ in the algebra $\mathfrak{A}^{(+)}$. We use (23) with $R_{e}$ replaced by $T_{\varepsilon}$ and first put $\alpha=1$, that is, let $y$ be in $\mathfrak{A}_{e}(1)$. If $x$ is also in $\mathfrak{A}_{\theta}(1), x y+y x$ is in $\mathfrak{A}_{e}(1)$ since $\mathfrak{A}_{e}(1)$ is a subring of $\mathfrak{A}^{(+)}$. But then $(x y+y x)\left(I-L_{e}\right)=0,(x y)\left(I-T_{e}\right)=0$, and $x y$ is in $\mathfrak{A}_{e}(1)$ by (23). We next let $x$ and $y$ be in $\mathfrak{A}_{\theta}(0), \alpha=0$ so that $x y+y x$ is in $\mathfrak{A}_{e}(0),(x y+y x) L_{e}=0,(x y) T_{e}=0$, and $x y$ is in $\mathfrak{A}_{e}(0)$ by (23). This proves that $\mathfrak{A}_{e}(1)$ and $\mathfrak{A}_{e}(0)$ are subrings of $\mathfrak{A}$. They have already been shown to be orthogonal.

The next result is obtained by putting $y=e$ in (29). Then $\alpha=1$. Take $x$ in $\mathfrak{A}_{e}(1 / 2)$ so that $x e+e x=x$. Then $x\left(I-L_{\theta}\right)=x-e x=x e=2 x e\left(I-T_{\theta}\right)$, $x e=2(x e) T_{e},(x e) T_{e}=x e / 2, x e$ is in $\mathfrak{A}_{e}(1 / 2)$. However $x$ is in $\mathfrak{A}_{e}(1 / 2)$ and so $e x=x-x e$ is in $\mathfrak{A}_{e}(1 / 2)$.

To obtain the relations (30) we let $y=e$ in (29) and let $x$ be in $\mathfrak{A}_{\theta}(1 / 2)$. Then $(x e) z+(z e) x=x(e z)+z(e x)$. However $x e=x-e x$ so that $x z+(z e) x$ $=x(e z)+z(e x)+(e x) z$. If $z$ is in $\mathfrak{A}_{0}(1)$ then $e z=z e=z$ and $x z=x z+z x-z(e x)$ $-(e x) z$. But $e x$ has already been shown to be in $\mathfrak{A}_{\odot}(1 / 2)$ and we may use (24) to see that both $x z+z x$ and $z(e x)+(e x) z$ are in $\mathfrak{A}_{e}(0)+\mathfrak{A}_{e}(1 / 2)$. It follows that both $x z$ and $z x$ are in $\mathfrak{A}_{e}(0)+\mathfrak{A}_{\odot}(1 / 2)$. We next put $e z=z e=0$ and obtain $x z=z(e x)+(e x) z$ which is in $\mathfrak{A}_{e}(1)+\mathfrak{A}_{e}(1 / 2)$ by the last relation of $(24), z x$ is also in this submodule, and we have proved (30).

We shall be particularly interested in those flexible rings having the property expressed by (26). We define a ring $\mathfrak{A}$ to be a stable ring if $\mathfrak{A}$ is a flexible power associative ring and

$$
\mathfrak{A}_{\Theta}(\lambda) \mathfrak{A}_{\Theta}(1 / 2) \leqq \mathfrak{A}_{\Theta}(1 / 2), \quad \mathfrak{A}_{\epsilon}(1 / 2) \mathfrak{A}_{\odot}(\lambda) \leqq \mathfrak{A}_{\odot}(1 / 2) \quad(\lambda=0,1),
$$

for every idempotent $e$ of $\mathfrak{A}$. We may then prove

Theorem 6. A flexible power associative ring $\mathfrak{A}$ is a stable ring if and only if $\mathfrak{A}^{(+)}$is stable.

For if $x y$ and $y x$ are in $\mathfrak{A}_{\epsilon}(1 / 2)$ for every $y$ of $\mathfrak{A}_{\odot}(1 / 2)$ and every $x$ of 
$\mathfrak{A}_{e}(1)+\mathfrak{A}_{e}(0)$ their sum $x y+y x$ is also in $\mathfrak{A}_{e}(1 / 2)$ and the commutative ring $\mathfrak{A}^{(+)}$is stable. Conversely let $\mathfrak{A}^{(+)}$be stable so that (26) holds. Put $y=e$ in (29) and obtain $(x e) z+(z e) x=x(e z)+z(e x)$ so that, if $x e=e x=\lambda x$, then $\lambda x z+(z e) x=x(e z)+\lambda z x$. Then

$$
\lambda x z+(z e+e z) x=[x(e z)+(e z) x]+\lambda z x .
$$

If $z$ is in $\mathfrak{A}_{e}(1 / 2)$ then $z e+e z=z, e z$ is in $\mathfrak{A}_{e}(1 / 2)$ by Theorem $5, w=x(e z)$ $+(e z) x$ is in $\mathfrak{A}_{e}(1 / 2)$ by the assumption that $\mathfrak{A}^{(+)}$is stable. Then $\lambda x z+(1-\lambda) z x$ is in $\mathfrak{A}_{e}(1 / 2)$. The value $\lambda=1$ yields $x z$ in $\mathfrak{A}_{e}(1 / 2)$ and the value $\lambda=0$ yields $z x$ in $\mathfrak{A}_{e}(1 / 2)$. But $x z+z x$ is in $\mathfrak{A}_{e}(1 / 2)$ and so both $x z$ and $z x$ are in $\mathfrak{A}_{e}(1 / 2)$ in every case, $\mathfrak{A}$ is stable.

It should be observed that when $\mathfrak{A}$ is a stable ring every subring $\mathfrak{B}$ of $\mathfrak{A}$ is stable. For $\mathfrak{B}$ is clearly flexible. If $e$ is an idempotent of $\mathfrak{B}$ we may write $\mathfrak{B}=\mathfrak{B}_{e}(1)+\mathfrak{B}_{e}(1 / 2)+\mathfrak{B}_{e}(0)$ and the definitions of the modules $\mathfrak{B}_{e}(\lambda)$ clearly imply that $\mathfrak{B}_{e}(\lambda) \leqq \mathfrak{A}_{e}(\lambda)$. But then the relations (31) imply corresponding relations for the modules $\mathfrak{B}_{e}(\lambda)$ and so $\mathfrak{B}$ is stable.

We note finally that when $\mathfrak{B}$ is an ideal of a stable algebra $\mathfrak{A}$, then $\mathfrak{A}_{0}=\mathfrak{A}-\mathfrak{B}$ is also stable. Indeed, let $e_{0}$ be an idempotent element of $\mathfrak{A}_{0}$. Then $e_{0}$ is a class $x+\mathfrak{B}$ where $x$ is necessarily a non-nilpotent element of $\mathfrak{A}, e_{0}^{k}=x^{k}+\mathfrak{B}=e_{0}$. The algebra $\mathfrak{E}=\mathfrak{F}[x]$ is an associative commutative non-nilpotent algebra and contains a principal idempotent $e$. We may write $\mathfrak{S}=\mathfrak{S}_{e}(1)+\mathfrak{S}_{e}(0)$ where the elements of $\mathfrak{S}_{e}(0)$ are nilpotent. If $e$ were in $\mathfrak{B}$ the algebra $\mathfrak{S}_{e}(1)=e \mathfrak{S}_{e}$ would be in $\mathfrak{B}$ and we would have $x+\mathfrak{B}=y+\mathfrak{B}$ where $y$ is in $\mathfrak{S}_{e}(0)$. But then $e_{0}=y+\mathfrak{B}$ could not be idempotent. Hence $e$ is not in $\mathfrak{B}, e+\mathfrak{B}=e(x)+\mathfrak{B}$ $=\alpha x+\mathfrak{B}$ for $\alpha \neq 0$ in $\mathfrak{F},(e+\mathfrak{B})^{2}=e^{2}+\mathfrak{B}=e+\mathfrak{B}=\alpha^{2} x^{2}+\mathfrak{B}, \alpha^{2} x^{2}+\mathfrak{B}=\alpha x+\mathfrak{B}$, $\alpha^{2}=\alpha, \alpha=1, e+\mathfrak{B}=e_{0}$. We conclude that the homomorphic mapping of $\mathfrak{A}$ on $\mathfrak{A}-\mathfrak{B}$ maps every element $z$ of $\mathfrak{A}$ such that $z e+e z=\lambda z$ on an element $z_{0}$ of $\mathfrak{A}-\mathfrak{B}$ such that $z_{0} e_{0}+e_{0} z_{0}=\lambda z_{0}$. Then the relations (31) for $\mathfrak{A}_{e}(\lambda)$ go into relations (31) for $\mathfrak{A}_{0 e_{0}}(\lambda)$ and so $\mathfrak{A}_{0}$ is also stable.

8. Principal idempotents of algebras. An idempotent $e$ of a ring $\mathfrak{A}$ is called a principal idempotent of $\mathfrak{A}$ if there is no idempotent $u$ of $\mathfrak{A}$ orthogonal to $e$. If $\mathfrak{A}$ is power-associative this means that $\mathfrak{A}_{e}(0)$ contains no idempotent. When we assume that $\mathfrak{A}$ is also a flexible algebra then $\mathfrak{A}_{e}(0)$ is a subalgebra of $\mathfrak{A}$ and so $e$ is principal if and only if $\mathfrak{A}_{e}(0)$ is a nilalgebra. We now make an additional assumption and prove

THeOREM 7. Let e be a principal idempotent of a stable algebra $\mathfrak{A}$. Then the elements of $\mathfrak{A}_{e}(1 / 2)$ are nilpotent.

The result is clearly only required for $\mathfrak{A}^{(+)}$since powers in $\mathfrak{A}^{(1)}$ in $\mathfrak{H}^{(+)}$ coincide. Hence it is sufficient to take the case where $\mathfrak{A}$ is commutative. We then use (14) with $x$ in $\mathfrak{A}_{e}(1 / 2)$ and operate on $e$ to obtain $e x^{3}=4(e x) x^{2}$ $-\left(e x^{2}\right) x-2[(e x) x] x$. But $e x=x / 2$ and so $e x^{3}=x^{3}-\left(e x^{2}\right) x$. By Theorem 2 we may write 


$$
w=x^{2}=w_{1}+w_{0}
$$

where $w_{0}$ is in $\mathfrak{X}_{e}(0)$ and $w_{1}$ is in $\mathfrak{U}_{e}(1)$ and use the property that $\mathfrak{A}$ is stable to see that $x^{3}=\left(w_{1}+w_{0}\right) x$ is in $\mathfrak{A}_{e}(1 / 2)$. Then $x^{3} / 2=x^{3}-w_{1} x, x^{2} x=2 w_{1} x=\left(w_{1}\right.$ $\left.+w_{0}\right) x$,

$$
w_{1} x=w_{0} x .
$$

We now let $e$ be principal so that $\mathfrak{A}_{e}(0)$ is a nilalgebra, $w_{0}^{k}=0$ for some positive integer $k$. Put $z=x^{2 k+1}$ and have $z^{2}=w^{2 k+1}=w_{1}^{2 k+1}+w_{0}^{2 k+1}$ since $w_{1}$ and $w_{0}$ are orthogonal. But $x^{2 k}=w_{0}^{k}+w_{1}^{k}$ is in $\mathfrak{A}_{e}(1)+\mathfrak{A}_{e}(0), z=x^{2 k+1}=x^{2 k} x$ is in $\mathfrak{A}_{e}(1 / 2), z^{2}=v_{1}+v_{0}$ where $v_{1}=w_{1}^{2 k+1}, v_{0}=w_{0}^{2 k+1}=0$. Hence we may apply (33) in this case, $v_{1} z=v_{0} z=0, z^{3}=\left(v_{1}+v_{0}\right) z=0, x^{6 k+3}=0, x$ is nilpotent. This concludes our study of the decomposition of a power-associative ring relative to an idempotent and we pass on now to the study of certain types of stable power-associative algebras.

\section{Chapter II. Trace-admissible algebras}

1. Admissible trace functions. We shall leave the theory of rings and turn to the theory of algebras considering only power-associative stable algebras over a field $\mathfrak{F}$. Let $\mathfrak{A}$ be such an algebra and call $\mathfrak{A}$ a trace-admissible algebra if there exists a function $\tau(x, y)$ with arguments $x$ and $y$ in $\mathfrak{A}$ and values in $\mathfrak{F}$ such that

$$
\begin{aligned}
& \text { I. } \tau(x, y) \text { is a bilinear function of } x \text { and } y \text {. } \\
& \text { II. } \tau(x, y)=\tau(y, x) \text {. } \\
& \text { III. } \tau(x y, z)=\tau(x, y z) \text {. } \\
& \text { IV. } \tau(x, y)=0 \text { if } x y \text { is nilpotent or zero. } \\
& \text { V. } \tau(e, e) \neq 0 \text { if } e \text { is an idempotent of } \mathfrak{A} \text {. }
\end{aligned}
$$

It should be noted that II and III imply

(1) $\quad \tau(x y, z)=\tau(y z, x)=\tau(z x, y)=\tau(x, y z)=\tau(y, z x)=\tau(z, x y)$.

We shall call any function $\tau(x, y)$ satisfying $\mathrm{I}-\mathrm{V}$ an admissible trace function for $\mathfrak{A}$. We now prove

Theorem 1. Let $\mathfrak{A}$ be a trace-admissible algebra and let $\mathfrak{N}_{\tau}$ be the set of all elements $x$ of $\mathfrak{A}$ such that $\tau(x, y)=0$ for every $y$ of $\mathfrak{A}$. Then $\mathfrak{N}_{\tau}$ is the nilradical $\mathfrak{N}$ of $\mathfrak{A}$.

For by $\mathrm{I}$ the set $\mathfrak{N}_{\tau}$ is a linear subspace of $\mathfrak{A}$. If $x$ is in $\mathfrak{R}_{\tau}$ and $y$ is in $\mathfrak{A}$, then $\tau(x y, z)=\tau(x, y z)$ by $(1), \tau(x, y z)=0$ for every $z, x y$ is in $\mathfrak{N}_{\tau}$. Similarly

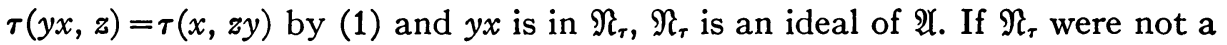
nilalgebra it would contain $\left(^{3}\right)$ an idempotent $e$ whereas $\tau(e, e) \neq 0$. Hence $\mathfrak{N}_{\tau} \leqq \mathfrak{N}$. Conversely if $x$ is in $\mathfrak{N}$ then $x y$ is in $\mathfrak{N}$ for every $x$ of $\mathfrak{A}, x y$ is nilpotent, $\tau(x, y)=0, \mathfrak{N} \leqq \mathfrak{N}_{\tau}, \mathfrak{R}=\mathfrak{N}_{\tau}$ as desired.

(3) Cf. $\S \mathrm{S}$ of the paper of footnote 2. 
We have now shown that $\mathfrak{N}_{\tau}$ is independent of $\tau$. Note also that if $\mathscr{B}$ is any subalgebra of $\mathfrak{A}$ and $\tau(x, y)$ is an admissible trace function for $\mathfrak{A}$ then $\tau(x, y)$ is also an admissible trace function for $\mathfrak{B}$. For clearly $\mathrm{I}-\mathrm{V}$ hold for $\mathfrak{B}$ when they hold for $\mathfrak{A}$. Also it has already been remarked that $\mathfrak{B}$ is stable when $\mathfrak{A}$ is.

2. The radical of an ideal. The radical of an ideal is completely determined by

THEOREM 2. Let $\mathfrak{B}$ be an ideal of a trace-admissible algebra $\mathfrak{A}$. Then the intersection of $\mathfrak{B}$ and the nilradical $\mathfrak{N}$ of $\mathfrak{A}$ is the nilradical $\mathfrak{N}_{0}$ of $\mathfrak{B}$.

For $\mathfrak{B} \cap \mathfrak{N}$ is a nilideal of $\mathfrak{B}, \mathfrak{B} \cap \mathfrak{N} \leqq \mathfrak{N}_{0}$. Conversely let $g$ be in $\mathfrak{R}_{0}$ so that either $g$ is in $\mathfrak{N}$ and hence in $\mathfrak{B} \cap \mathfrak{N}$ or there exists an element $x$ of $\mathfrak{A}$ such that $\tau(g, x) \neq 0$. Then $y=g x$ is not nilpotent and there exist elements $\alpha_{1}, \cdots, \alpha_{t}$ in $\mathfrak{F}$ such that $e=\alpha_{1} y \cdots \alpha_{t} y^{t}$ is idempotent. Now $\tau(e, e)=\sum_{t, j=1}^{t} \alpha_{i} \alpha_{j} \tau\left(y^{i}, y^{j}\right)$ $\neq 0$ and it follows that $\tau\left(y^{i}, y^{j}\right) \neq 0$ for some integers $i$ and $j$. However by (1) we have $\tau\left(y^{i}, y^{j}\right)=\tau\left(y^{i+j-1}, y\right)=\tau\left(y^{i+j-1}, g x\right)=\tau\left(g, x y^{i+j-1}\right)=0$ since $y$ is in $\mathfrak{B}, x y^{i+j-1}$ is in $\mathfrak{B}, g$ is in $\mathfrak{N}_{0}, g\left(x y^{i+j-1}\right)$ is in $\mathfrak{N}_{0}$ and is nilpotent. This proves that $\mathfrak{N}_{0} \leqq \mathfrak{B} \cap \mathfrak{N}, \mathfrak{R}_{0}=\mathfrak{B} \cap \mathfrak{N}$.

We define a trace-admissible algebra $\mathfrak{A}$ to be semi-simple if its nilradical $\mathfrak{N}=0$ and immediately have

THEOREM 3. Every ideal of a semi-simple trace-admissible algebra is semisimple.

3. The nilradical in the Pierce decomposition. We now refer to the decomposition $\mathfrak{A}=\mathfrak{A}_{e}(1)+\mathfrak{U}_{e}(1 / 2)+\mathfrak{A}_{e}(0)$ and prove

Theorem 4. The nilradical $\mathfrak{R}_{\lambda}$ of $\mathfrak{A}_{e}(\lambda)$ is the intersection of $\mathfrak{A}_{e}(\lambda)$ and the nilradical $\mathfrak{N}$ of $\mathfrak{A}$ for $\lambda=0,1$.

For $\mathfrak{R} \cap \mathfrak{A}_{e}(\lambda)$ is evidently a nilideal of $\mathfrak{A}_{e}(\lambda)$ and is contained in $\mathfrak{R}$. Conversely if $x$ is in $\mathfrak{N}$ then $x a_{\lambda}$ is nilpotent for every $a_{\lambda}$ of $\mathfrak{A}_{e}(\lambda)$ and so $\tau\left(x, a_{\lambda}\right)=0$. Also $\tau\left(x, a_{1-\lambda}\right)=0$ for every $a_{1-\lambda}$ of $\mathfrak{A}_{e}(1-\lambda)$ since $x a_{1-\lambda}=0$. Finally if $b$ is in $\mathfrak{A}_{e}(1 / 2)$ then $\tau(x, b)=\tau(x, e b+b e)=\tau(x, e b)+\tau(x, b e)=\tau(x e, b)+\tau(e x, b)$ $=2 \lambda \tau(x, b), \tau(x, b)=0$ if $\lambda=0,1$. Hence $\tau(x, a)=0$ for every $a$ of $\mathfrak{A}, x$ is in $\mathfrak{N} \cap \mathfrak{A}_{e}(\lambda), \mathfrak{N}_{\lambda}=\mathfrak{N} \cap \mathfrak{A}_{e}(\lambda)$.

In the case of a principal idempotent we may prove

Theorem 5. Let e be a principal idempotent of a stable algebra $\mathfrak{A}$. Then $\mathfrak{A}_{e}(1 / 2)+\mathfrak{A}_{e}(0)$ is contained in the nilradical of $\mathfrak{A}$.

For $\mathfrak{A}_{e}(0) \leqq \mathfrak{N}$ by Theorem 4 and the property that the maximal nilideal of $\mathfrak{A}_{e}(0)$ is $\mathfrak{A}_{e}(0)$ itself. By Theorem 1.7 , if $b$ is in $\mathfrak{A}_{e}(1 / 2)$ then $b$ is nilpotent. It follows that $b^{2},(b+c)^{2}$, and $c^{2}$ are nilpotent for every $b$ and $c$ of $\mathfrak{A}_{\epsilon}(1 / 2)$. Then $\tau(b+c, b+c)=0=\tau(b, b)+\tau(c, c)+2 \tau(b, c), \tau(b, c)=0$. But by the proof of Theorem $4, \tau(b, x)=0$ for every $x$ in $\mathfrak{A}_{e}(0)+\mathfrak{A}_{e}(1), b$ is in $\mathfrak{R}, \mathfrak{A}_{e}(1 / 2) \leqq \mathfrak{N}$. 
An algebra $\mathfrak{A}$ which is not a nilalgebra has an idempotent and hence a principal idempotent. Applying Theorem 5 we have

TheOREM 6. A semi-simple trace-admissible algebra has a unity element.

As a consequence of Theorems 3 and 6 we now have

TheORem 7. Let $\mathfrak{B}$ be an ideal of a trace-admissible semi-simple algebra $\mathfrak{A}$. Then $\mathfrak{A}=\mathfrak{B} \oplus \mathfrak{C}, \mathfrak{B}$ has a unity element $e$ and $\mathfrak{E}=\mathfrak{A}_{\Theta}(0)$.

For $\mathfrak{B}$ has a unity element $e$ by Theorems 3 and 6 , and if $x$ is in $\mathfrak{U}_{e}(1 / 2)$ we have $x e+e x=x$ is in $\mathfrak{B}, x e+e x=x, 2 x=x, x=0, \mathfrak{A}_{e}(1 / 2)=0, \mathfrak{A}=\mathfrak{B} \oplus \mathfrak{A}_{e}(0)$.

Theorem 7 implies that every semi-simple trace-admissible algebra $\mathfrak{A}$ is a direct sum of simple algebras which are not nilalgebras.

\section{Chapter III. Commutative shrinkable algebras}

1. Algebras of level one. Let $\mathfrak{A}$ be any algebra over a field $\mathfrak{F}$ so that the multiplications of $\mathfrak{A}$ are linear transformations $R_{x}$ and $L_{x}$ over $\mathfrak{F}$ of $\mathfrak{A}$. Use the notation $T(x)$ to represent either multiplication and the notation $T\left(x_{1} \cdots x_{8}\right)$ to represent a multiplication $T(x)$ defined for a product $x=x_{1} \cdots x_{8}$ of the factors $x_{1}, \cdots, x_{8}$ with an unspecified association. Then we shall call $\mathfrak{A}$ a shrinkable algebra of shrinkability level $s$ if every $T\left(x_{1} \cdots x_{s+1}\right)$ is identically equal to a finite sum of products $\lambda T_{1} \cdots T_{r}$ with fixed coefficients $\lambda$ in $\mathfrak{F}$, where the transformations $T_{i}$ are multiplications $T_{i}\left(z_{i}\right)$ defined for products $z_{i}$ of $t_{i}$ factors $x_{k}$, and $t_{1}+\cdots+t_{r}=s+1$. We propose to investigate here the relations possible for commutative power-associative shrinkable algebras of levels one and two over $\mathfrak{F}$ of characteristic not two or three.

A commutative algebra $\mathfrak{A}$ of level one has the property that $R_{x y}=\lambda R_{x} R_{y}$ $+\mu R_{y} R_{x}$ for every $x$ and $y$ of $\mathfrak{A}$ where $\lambda$ and $\mu$ are fixed elements of $\mathfrak{F}$. Since $x y=y x$ we also have $R_{x y}=R_{y x}=\lambda R_{y} R_{x}+\mu R_{x} R_{y}$. Then $2 R_{x y}=(\lambda+\mu)\left(R_{x} R_{y}\right.$ $\left.+R_{y} R_{x}\right)$ and, since $\mathfrak{F}$ does not have characteristic two, $R_{x y}=\nu\left(R_{x} R_{y}+R_{y} R_{x}\right)$. It follows that $R_{x x}=2 \nu R_{x}^{2}$ is commutative with $R_{x}$ and that $\mathfrak{A}$ is a Jordan algebra.

However we may analyze the algebras of level one more deeply. We have $a(x y)=\nu(a x) y+\nu(a y) x$ and so $\nu R_{a x}=R_{x} R_{a}-\nu R_{a} R_{x}=\nu^{2}\left(R_{x} R_{a}+R_{a} R_{x}\right)$. Then

$$
\left(\nu^{2}+\nu\right) R_{a} R_{x}=\left(1-\nu^{2}\right) R_{x} R_{a} .
$$

If $\nu=0$ then $R_{x} R_{a}=0$ for every $x$ and $a$ of $\mathfrak{A}$ and $R_{x a}=0=R_{x} R_{a}, \mathfrak{A}$ is an associative nilpotent commutative algebra in which all products of three elements are zero. If $\nu=1$ then $R_{a} R_{x}=0$ and we have the same result as in the case $\nu=0$. When $\nu \neq 0$ and $\nu^{2} \neq 1$ then $R_{a} R_{x}=\nu^{-1}(1-\nu) R_{x} R_{a}, \nu\left(R_{y} R_{x}+R_{x} R_{y}\right)$ $=(1-\nu+\nu) R_{x} R_{y}=R_{x} R_{y}=R_{x y}$ and $\mathfrak{A}$ is a commutative associative algebra.

There remains the case where $\nu=-1$ and so $R_{x y}=-\left(R_{x} R_{y}+R_{y} R_{x}\right)$, $R_{x x}=-2 R_{x}^{2}, x^{3}+2 x^{3}=3 x^{3}=0$. Then the Jordan algebra is a nilalgebra and is known to be nilpotent. We have proved 
THEOREM 1. A commutative shrinkable algebra of level one is either associative or is a nilpotent Jordan algebra satisfying the relation $x(y z)+y(z x)+z(x y)$ $=0$.

2. Algebras of level two. The general relation satisfied by a commutative shrinkable algebra of level two expresses $R_{(x y) z}$ as a sum of terms of the form $\lambda R_{x y} R_{z}, \mu R_{z} R_{x y}$ and $\nu R_{x} R_{y} R_{z}$ with $\lambda, \mu, \nu$ in $\mathfrak{F}$. If we use the hypothesis $x y=y x$ to see that $R_{(x y) z}+R_{(y x) z}=2 R_{(x y) z}$ we shall obtain a relation expressing $R_{(x y) s}$ as a sum of products unaltered by the interchange of $x$ and $y$. The relation must then have the form

$$
R_{(x y) z}=\lambda_{1} R_{x y} R_{z}+\lambda_{2}\left(R_{x z} R_{y}+R_{y z} R_{x}\right)+G+H,
$$

where

$$
G=\mu_{1} R_{z} R_{x y}+\mu_{2}\left(R_{y} R_{x z}+R_{x} R_{y z}\right)
$$

and

$$
\begin{aligned}
H= & \nu_{1} R_{z}\left(R_{x} R_{y}+R_{y} R_{x}\right)+\nu_{2}\left(R_{x} R_{y}+R_{y} R_{x}\right) R_{z} \\
& +\nu_{3}\left(R_{x} R_{z} R_{y}+R_{y} R_{z} R_{x}\right),
\end{aligned}
$$

for coefficients $\lambda_{1}, \lambda_{2}, \mu_{1}, \mu_{2}, \nu_{1}, \nu_{2}, \nu_{3}$ in $\mathfrak{F}$.

If $w$ is in $\mathfrak{A}$ we form $w[(x y) z]$ and use (2) to write

$$
\begin{aligned}
w[(x y) z]= & \lambda_{1}[w(x y)] z+\lambda_{2}[w(x z)] y+\lambda_{2}[w(y z)] x+\mu_{1}(w z)(x y) \\
& +\mu_{2}(w x)(y z)+\mu_{2}(w y)(x z)+\nu_{1}[(w z) x] y+\nu_{1}[(w z) y] x \\
& +\nu_{2}[(w x) y+(w y) x] z+\nu_{3}[(w x) z] y+\nu_{3}[(w y) z] x .
\end{aligned}
$$

We may interchange the symbols $w$ and $z$ and write the result as a transformation on $w$ to obtain the relation

$$
\begin{aligned}
R_{x y} R_{z}= & \lambda_{1} R_{(x y) z}+\lambda_{2}\left(R_{x} R_{z} R_{y}+R_{y} R_{z} R_{x}\right)+G+\nu_{1} R_{z}\left(R_{x} R_{y}+R_{y} R_{x}\right) \\
& +\nu_{2}\left[R_{(x z) y}+R_{(y z) x}\right]+\nu_{3}\left(R_{x z} R_{y}+R_{y z} R_{x}\right) .
\end{aligned}
$$

The elimination of the common term $G+\nu_{1} R_{z}\left(R_{x} R_{y}+R_{y} R_{x}\right)$ between (6) and (2) then yields

$$
\begin{aligned}
\left(1+\lambda_{1}\right) R_{(x y) z} & +\nu_{2} R_{(x z) y}+\nu_{2} R_{(y z) x} \\
= & \left(1+\lambda_{1}\right) R_{x y} R_{z}+\left(\lambda_{2}-\nu_{3}\right)\left(R_{x z} R_{y}+R_{y z} R_{x}\right) \\
& -\left(\lambda_{2}-\nu_{3}\right)\left(R_{x} R_{z} R_{y}+R_{y} R_{z} R_{x}\right)+\nu_{2}\left(R_{x} R_{y}+R_{y} R_{x}\right) R_{z} .
\end{aligned}
$$

We may finally interchange the symbols $x$ and $z$ as well as the symbols $y$ and $w$ in (5) and write the result as an equation in transformations on $w$ as

$$
\begin{gathered}
R_{z} R_{x} R_{y}=\lambda_{1} R_{z} R_{y} R_{x}+\lambda_{2}\left[R_{(x z) y}+R_{x} R_{y} R_{z}\right]+G+\nu_{1}\left[R_{(x y) z}+R_{x y} R_{z}\right] \\
+\nu_{2}\left(R_{y z} R_{x}+R_{y} R_{z} R_{x}\right)+\nu_{3}\left[R_{(y z) x}+R_{y} R_{x} R_{z}\right] .
\end{gathered}
$$


At this point we shall further restrict our study. It should be clear that there is nothing to say about algebras of level two which are also of level one and so we may assume that our algebras do not have level one. Moreover a study of those classes of commutative algebras containing no algebras with a unity quantity should be of only secondary interest in this first investigation of the types of shrinkable algebras. Thus we shall attempt to determine the values of our parameters only for classes of power-associative algebras containing algebras with a unity quantity $e$ and not of level one. For such algebras $R_{e}$ is the identity transformation and a relation of the form

$$
\alpha R_{x y}+\beta R_{x} R_{y}+\gamma R_{y} R_{x}=0
$$

is possible only when $\alpha=0$.

Let us now apply this property. Substitute first $z=e$ and then $x=e$ in (2) to obtain

$$
\lambda_{1}+\mu_{1}=\lambda_{2}+\mu_{2}=1 .
$$

Make the same substitution in (6) and (7) and obtain

$$
\begin{gathered}
2 \nu_{2}=0, \quad 1+\lambda_{1}=\lambda_{2}-\nu_{3}, \\
\lambda_{2}+\mu_{1}+2 \nu_{1}+\nu_{3}=0, \\
\lambda_{2}+\mu_{2}+\nu_{1}+\nu_{3}=0 .
\end{gathered}
$$

Then $\nu_{2}=0$,

$$
\nu_{1}+\nu_{3}=-1, \quad \lambda_{2}+\mu_{1}+\nu_{1}=1 .
$$

If $\lambda_{1}+1=0$ we may use ( 7$)$ and the fact that $\nu_{2}=0$ to see that the corresponding class of algebras is defined by a relation of the form (2) with $\mu_{1}=\mu_{2}=0$. Then the study we have made yields (10) and (11) and thus $\lambda_{1}=\lambda_{2}=1, \nu_{3}=-1, \nu_{1}=\nu_{2}=0$. The resulting identity is $R_{(x y) z}=R_{x y} R_{z}+R_{x z} R_{y}$ $+R_{y z} R_{x}-\left(R_{x} R_{z} R_{y}+R_{y} R_{z} R_{x}\right)$ and our algebras are known to be Jordan algebras.

There remains the case $\lambda_{1}=-1$. In this case $\lambda_{2}=\nu_{3}$ and $\mu_{1}=2$. Then we have the relation

$$
\begin{aligned}
R_{(x y) z}= & 2 R_{z} R_{x y}+R_{y} R_{x z}+R_{x} R_{y z}-R_{x y} R_{z}-R_{z}\left(R_{x} R_{y}+R_{y} R_{x}\right) \\
& +\nu_{3}\left[\left(R_{x} R_{z} R_{y}+R_{y} R_{z} R_{x}\right)-R_{z}\left(R_{x} R_{y}+R_{y} R_{x}\right)\right. \\
& \left.+\left(R_{x z} R_{y}+R_{y z} R_{x}\right)-\left(R_{y} R_{x z}+R_{x} R_{y z}\right)\right] .
\end{aligned}
$$

Compute $R_{(y z) x}$ and $R_{(x z) y}$ using (13) and add the three formulas so obtained to give

$$
P=2 \nu_{3}\left[\left(R_{x y} R_{z}+R_{y z} R_{x}+R_{z x} R_{y}\right)-\left(R_{z} R_{x y}+R_{x} R_{y z}+R_{y} R_{z x}\right)\right]
$$

where 


$$
\begin{aligned}
P= & R_{(x y) z}+R_{(z x) y}+R_{(y z) x}-4\left(R_{z} R_{x y}+R_{y} R_{x z}+R_{x} R_{y z}\right) \\
& +\left(R_{x y} R_{z}+R_{y z} R_{x}+R_{z x} R_{y}\right) \\
& +\left[R_{x}\left(R_{y} R_{z}+R_{z} R_{y}\right)+R_{y}\left(R_{x} R_{z}+R_{z} R_{x}\right)+R_{z}\left(R_{x} R_{y}+R_{y} R_{x}\right)\right]
\end{aligned}
$$

We now apply the assumption that $\mathfrak{A}$ is a power-associative commutative algebra. By (1.13), this is precisely the condition $P=0$ and formula (14) for $\nu_{3} \neq 0$ yields a standard identity for Jordan algebras. The only remaining case is thus the case $\nu_{3}=0$ in (13) and we have proved

THEOREM 2. Let $\mathfrak{A}$ be a commutative power-associative algebra over a field of characteristic not two or three and let $\mathfrak{A}$ have shrinkability level two and belong to a class of such algebras containing algebras with a unity quantity. Then $\mathfrak{A}$ is either a Jordan algebra or an algebra satisfying the relation

$$
R_{(x y) z}=2 R_{z} R_{x y}+R_{y} R_{x z}+R_{x} R_{y z}-R_{x y} R_{z}-R_{z}\left(R_{x} R_{y}+R_{y} R_{x}\right) .
$$

We shall refer to algebras defined by (15) as static algebras and shall obtain a structure theory for such algebras in the remaining sections of this chapter.

3. Static algebras. The identity (15) is equivalent to

$$
\begin{aligned}
{[(x y) z] w+[(x y) w] z+[(z w) x] y+} & {[(z w) y] x } \\
& =2(x y)(z w)+(x z)(y w)+(y z)(x w),
\end{aligned}
$$

a relation invariant under permutations of a transitive group of order eight on the four letters $x, y, z, w$. Put $y=x$ and $w=z$ to obtain $2\left(x^{2} z\right) z+2\left(z^{2} x\right) x$ $=2 x^{2} z^{2}+2(x z)^{2}$. Then we have proved one of the implications in the following

THEOREM 3. Let $\mathfrak{A}$ be a commutative algebra over a field $\mathfrak{F}$ of characteristic not two. Then $\mathfrak{A}$ is a static algebra if and only if

$$
x^{2} y^{2}+(x y)^{2}=x\left(x y^{2}\right)+y\left(y x^{2}\right)
$$

for every $x$ and $y$ of $\mathfrak{A}$.

The remaining implication, (17) implies (16), is derived by first replacing $y$ by $z+\lambda w$ in (17) and then equating the coefficients of $\lambda$. This yields $2 x^{2}(z w)$ $+2(x z)(x w)=2 x[x(z w)]+z\left(w x^{2}\right)+w\left(z x^{2}\right)$. The replacement of $x$ by $x+\mu y$ and the equality of the coefficients of $\mu$ will then yield (16).

The relation (17) evidently implies that $x^{2} x^{2}=x\left(x x^{2}\right)=\left(x^{2} x\right) x$ and so the commutative algebras which we are calling static algebras are necessarily power-associative.

4. Solvable static algebras. If $\mathfrak{B}$ is any subalgebra of an algebra $\mathfrak{A}$ we shall designate by $\mathfrak{B}^{*}$ the associative algebra generated by the multiplications $R_{b}, L_{b}$ of $\mathfrak{A}$ which are defined for all quantities $b$ of $\mathfrak{B}$. Every solvable subalgebra $\mathfrak{B}$ contains a maximal proper subalgebra $\mathbb{E}$, such that $\mathbb{E}$ contains the product of any two elements of $\mathfrak{B}, \mathfrak{B}-\mathfrak{C}$ is a zero algebra of order one, 
$\mathfrak{B}=\mathfrak{C}+w \mathfrak{F}$. Define $\mathfrak{E}=\mathfrak{B}^{*} \mathfrak{C}^{*}+\mathfrak{C}^{*}$ and let $\mathfrak{A}$ be a static algebra. Then we may prove

LEMMA 1. Let $x$ be in $\mathfrak{B}$ and $c$ be in $\mathfrak{E}$. Then $R_{z} R_{x}^{2}$ is in $\mathfrak{S}$.

For $R_{(x x) c}=2 R_{c} R_{x x}+2 R_{x} R_{c x}-R_{x x} R_{c}-2 R_{c} R_{x}^{2}$. Then $(x x) c, x x, c x, c$ are all in $\mathfrak{E},-2 R_{c} R_{x}^{2}$ is in $\mathfrak{E}$, and we use our basic assumption that $\mathfrak{F}$ does not have characteristic two.

We next prove

Lemma 2. Let $x$ be in $\mathfrak{B}$ and $c$ and $d$ be in $\mathfrak{S}$. Then $R_{c} R_{d} R_{x}$ is in $\mathfrak{W}$.

For $R_{(x d) c}=2 R_{c} R_{x d}+R_{d} R_{x c}+R_{x} R_{c d}-R_{x d} R_{c}-R_{c}\left(R_{x} R_{d}+R_{d} R_{x}\right)$ and our result follows from the fact that $(x d) c, x d, x c, c d, c, d$ are all in $\Subset$.

As in all cases of power-associative commutative algebras we have the relation

$$
R_{(x x) x}=4 R_{x} R_{x x}-R_{x x} R_{x}-2 R_{x}^{3}
$$

Then

$$
R_{x} R_{(x x) x}=4 R_{x}^{2} R_{x x}-R_{x} R_{x x} R_{x}-2 R_{x}^{4}
$$

and

$$
R_{(x x) x} R_{x}=4 R_{x} R_{x x} R_{x}-R_{x x} R_{x}^{2}-2 R_{x}^{4}
$$

It follows that

$$
R_{x} R_{(x x) x}-R_{(x x) x} R_{x}=4 R_{x}^{2} R_{x x}+R_{x x} R_{x}^{2}-5 R_{x} R_{x x} R_{x} .
$$

Then

$$
\begin{aligned}
R_{\left(x^{2} x\right) x}= & 2 R_{x} R_{(x x) x}+R_{x x}^{2}+R_{x} R_{(x x) x}-R_{(x x) x} R_{x} \\
& -R_{x}\left(R_{x} R_{x x}+R_{x x} R_{x}\right) \\
= & 2 R_{x} R_{x x x}+R_{x x}^{2}+R_{x x} R_{x}^{2}+3 R_{x}^{2} R_{x x}-6 R_{x} R_{x x} R_{x} .
\end{aligned}
$$

By Lemma $1, R_{x x} R_{x}^{2}$ is in $\mathfrak{W}$. But then $6 R_{x} R_{x x} R_{x}$ is in $\mathfrak{W}$ and so $R_{x} R_{x x} R_{x}$ is in $\mathfrak{S}$. By (19) we have

Lemma 3. If $x$ is in $\mathfrak{B}$ then $R_{x}^{4}$ is in $\mathfrak{E}$.

Since $R_{x}^{4}$ is in $\mathfrak{S}$ for every $x$ of $\mathscr{B}$ it will be true that $\left(R_{x+\lambda y}\right)^{4}=R_{x}^{4}+\lambda S$ $+\lambda^{2} T+\lambda^{3} V+\lambda^{4} R_{y}^{4}$ will be in $\mathfrak{S}$ for every $x$ and $y$ of $\mathfrak{B}$. Then $\lambda S+\lambda^{2} T+\lambda^{3} V$ will be in $\mathfrak{S}$ and, since we are assuming always that the field $\mathfrak{F}$ has characteristic prime to six, $S$ is in $\mathfrak{S}$. But $S=R_{x}^{2}\left(R_{x} R_{y}+R_{y} R_{x}\right)+\left(R_{y} R_{x}+R_{x} R_{y}\right) R_{x}^{2}$. Assume now that $y$ is in (S so that $R_{x}^{2} R_{x} R_{y}$ is in $\mathfrak{S}$. By Lemma $1, R_{y} R_{x}^{2}$ is in $\mathfrak{Y}$ and, since $\mathfrak{B}^{*} \mathfrak{W} \leqq \mathfrak{W}$, we see that $R_{x} R_{y} R_{x}^{2}$ is in $\mathfrak{S}$. Also $2 R_{x}^{3}=4 R_{x} R_{x x}-R_{x x} R_{x}-R_{(x x) x}$ 
and so

$$
2 R_{y} R_{x}^{3}=4 R_{y} R_{x} R_{x x}-R_{y} R_{x x} R_{x}-R_{y} R_{(x x) x} .
$$

This transformation is in $\mathfrak{g}$ since $R_{y} R_{x x} R_{x}$ is in $\mathfrak{g}$ by Lemma 2. Then $R_{y} R_{x}^{3}$ is in $\mathfrak{E}$ and we have proved that $S, R_{x}^{3} R_{y}, R_{y} R_{x}^{3}, R_{x} R_{y} R_{x}^{2}$ are all in $\mathfrak{E}$. This yields

Lemma 4. Let $x$ be in $\mathfrak{B}$ and $c$ be in $\mathfrak{E}$. Then $R_{x}^{2} R_{c} R_{x}$ and $R_{c} R_{x}^{3}$ are in $\mathfrak{g}$.

We finally compute $R_{x(c x)}=3 R_{x} R_{c x}+R_{c} R_{x x}-R_{c x} R_{x}-R_{x}\left(R_{c} R_{x}+R_{x} R_{c}\right)$ and form the product

$$
R_{d} R_{x(c x)}=3 R_{d} R_{x} R_{c x}+R_{d} R_{c} R_{x x}-R_{d} R_{c x} R_{x}-R_{d} R_{x} R_{c} R_{x}-R_{d} R_{x}^{2} R_{c} .
$$

By Lemma 2 the term $R_{d} R_{c x} R_{x}$ is in $\mathfrak{W}$. All other terms have a right-hand factor $R_{y}$ with $y$ in $\mathbb{S}$ and we have proved

Lemma 5. Let $x$ be in $\mathfrak{B}$ and $c$ and $d$ be in $\mathfrak{E}$. Then $R_{d} R_{x} R_{c} R_{x}$ is in $\mathfrak{G}$.

We may now prove that $\left(\mathfrak{B}^{*}\right)^{4} \leqq \mathfrak{S}$. It is evidently sufficient to prove that $P=R_{x} R_{y} R_{z} R_{u}$ is in $\mathfrak{S}$ for all $x, y, z, u$ of $\mathfrak{B}$ and since $P$ is linear in $x, y, z, u$ it is sufficient to take the symbols $x, y, z, u$ to be either in $\mathbb{S}$ or equal to $w$ in $\mathfrak{B}$. If $u$ is in $\mathfrak{S}$ then $\mathfrak{S}$ contains $P$ so we may take $u=w$. If $z$ and $y$ are in $\mathbb{S}$ then $P$ is in $\mathfrak{S}$ by Lemma 2. If $z$ is in $\mathfrak{S}$ but $y=w$ then either $x$ is in $\mathfrak{S}$ and $P$ is in $\mathfrak{S}$ by Lemma 5 or $x=w, P=R_{w}^{2} R_{y} R_{w}$ is in $\mathfrak{S}$ by Lemma 4 . There remains the case $z=u=w, P=R_{x} R_{y} R_{w}^{2}$. If $y$ is in (S) then $P$ is in $\mathfrak{S}$ by Lemma 1 . Otherwise $P=R_{x} R_{w}^{3}$ and either $x=w$ and $P=R_{w}^{4}$ is in $\mathfrak{S}$ by Lemma 3 or $x$ is in $\mathcal{E}, P$ is in $\mathfrak{S}$ by Lemma 4 . This completes our argument and implies that $\left(\mathfrak{B}^{*}\right)^{5}$ $\leqq \mathfrak{B}^{*}\left(\mathfrak{B}^{*} \mathfrak{C}^{*}+\mathfrak{C}^{*}\right) \leqq \mathfrak{B}^{*} \mathfrak{C}^{*}$. An immediate induction implies that $\left(\mathfrak{B}^{*}\right)^{4 k+1}$ $\leqq \mathfrak{B}^{*}\left(\mathfrak{S}^{*}\right)^{k}$.

If $\mathfrak{B}$ is a solvable subalgebra of order one of a static algebra $\mathfrak{A}$ then $\mathfrak{B}$ $=w \mathfrak{F}, w^{2}=0=w^{3}$. By $(18)$ we see that $R_{w}^{3}=0$. Since $\mathfrak{B}^{*}=\mathfrak{F}\left[R_{w}\right]$ we see that $\mathfrak{B}^{*}$ is nilpotent. Let us assume then that $\mathfrak{B}$ has order $m$ and that the property that $\mathfrak{B}$ is solvable implies that $\mathfrak{B}^{*}$ is nilpotent is valid for solvable subalgebras of order $m-1$ of static algebras. Then the algebra $\mathbb{E}$ of the argument above is solvable and has order $m-1$, $\mathfrak{C}^{*}$ is nilpotent, $\left(\mathfrak{S}^{*}\right)^{k}=0$ for some positive integer $k,\left(\mathfrak{B}^{*}\right)^{4 k+1}=0, \mathfrak{B}^{*}$ is nilpotent. We have proved

TheOREM 4. Let $\mathfrak{B}$ be a solvable subalgebra of a static algebra $\mathfrak{A}$ over a field $\mathfrak{F}$ of characteristic not two or three. Then $\mathfrak{B}^{*}$ is nilpotent.

As in the theory of Jordan algebras we have the immediate

Corollary. A solvable static algebra is nilpotent.

5. The radical of a static algebra. We shall define the radical of a static algebra $\mathfrak{A}$ to be the maximal nilideal $\mathfrak{N}$ of $\mathfrak{A}$, and shall call $\mathfrak{A}$ semi-simple if $\mathfrak{N}=0$. Then we may prove 
TheOREM 5. Let $\mathfrak{A}$ be a static algebra over a nonmodular field $\mathfrak{F}$. Then the radical of $\mathfrak{A}$ is the set $\mathfrak{N}_{0}$ of all quantities $x$ of $\mathfrak{A}$ such that the trace $\tau\left(R_{x y}\right)=0$ for every $y$ of $\mathfrak{A}$.

For $\mathfrak{R}_{0}$ is clearly a linear space over $\mathfrak{F}$. To prove that $\mathfrak{R}_{0}$ is an ideal, we need only show that whenever $x$ is in $\mathfrak{R}_{0}$ and $y$ is in $\mathfrak{A}$, then $R_{(x y) z}-R_{x(y z)}$ has trace zero for every $z$ in $\mathfrak{A}$. This follows from (15) since

$$
R_{(y z) x}=2 R_{x} R_{y z}+R_{y} R_{x z}+R_{z} R_{x y}-R_{y z} R_{x}-R_{x}\left(R_{y} R_{z}+R_{z} R_{y}\right),
$$

and the trace of the transformation

$$
\begin{aligned}
R_{(x y) z}-R_{x(y z)}= & \left(R_{y z} R_{x}-R_{x} R_{y z}\right)+\left(R_{z} R_{x y}-R_{x y} R_{z}\right) \\
& +\left[\left(R_{x} R_{y}\right) R_{z}-R_{z}\left(R_{x} R_{y}\right)\right]+\left[R_{x}\left(R_{z} R_{y}\right)-\left(R_{z} R_{y}\right) R_{x}\right]
\end{aligned}
$$

evidently vanishes. If $\mathfrak{R}_{0}$ were not a nilideal it would contain an idempotent $e=e^{2}$ and we would have $\tau\left(R_{e e}\right)=0$ whereas $\mathfrak{A}_{e}(1)$ contains $e$ and is not zero, the characteristic roots of $R_{e}$ are $1,1 / 2,0$ and are not all zero, $\tau\left(R_{e}\right)>0$. Hence $\mathfrak{N}_{0} \leqq \mathfrak{N}$. But if $x$ is in $\mathfrak{N}$ then $z=x y$ is in $\mathfrak{N}$ and is a nilpotent quantity, $\mathfrak{B}=\mathfrak{F}[z]$ is an associative algebra defined by a nilpotent quantity and so is solvable, $\mathfrak{B}^{*}$ is nilpotent, $R_{z}$ is nilpotent, $\tau\left(R_{z}\right)=0, x$ is in $\mathfrak{N}_{0}$. Hence $\mathfrak{N}_{0}=\mathfrak{N}$.

6. Decomposition relative to an idempotent. Since a static algebra $\mathfrak{A}$ is a commutative algebra we see that

$$
\mathfrak{U}=\mathfrak{A}_{e}(1)+\mathfrak{A}_{e}(1 / 2)+\mathfrak{U}_{e}(0)
$$

where $\mathfrak{A}_{e}(1)$ and $\mathfrak{U}_{e}(0)$ are orthogonal algebras. Put $y=w=e$ in $(16)$ and obtain

$$
[(x e) z] e+[(x e) e] z+[x(e z)] e+[e(e z)] x=3(x e)(z e)+(x z) e .
$$

If $x$ and $z$ are in $\mathfrak{A}_{e}(1 / 2)$ then $x e=x / 2, z e=z / 2$ and (26) yields $(x z) e / 2$ $+x z / 4+(x z) e / 2+x z / 4=3 x z / 4+(x z) e, x z / 4=0, x z=0$. Thus $\mathfrak{A}_{e}(1 / 2)$ is a zero algebra. Put $x e=x, z e=z / 2$ and have $(x z) e+x z+(x z) e / 2+x z / 4=3 x z / 2$ $+(x z) e$ and thus $(x z)\left(2 R_{e}-I\right)=0, x z$ is in $\mathfrak{A}_{e}(1 / 2)$. Finally put $x e=0, z e=z / 2$ and obtain $(x z) e / 2+x z / 4=(x z) e,(x z)\left(2 R_{e}-I\right)=0$. We have proved

Theorem 6. Let $e$ be an idempotent of a static algebra $\mathfrak{A}$. Then $\mathfrak{A}_{\boldsymbol{e}}(1 / 2)$ is an ideal of $\mathfrak{A}$ and is a zero algebra.

7. Structure of semi-simple static algebras. It follows from Theorem 6 that if a static algebra $\mathfrak{A}$ contains no solvable ideals and if $e$ is an idempotent of $\mathfrak{A}$ then $\mathfrak{A}$ is the direct sum of $\mathfrak{A}_{e}(1)$ and $\mathfrak{A}_{e}(0)$. Let us assume now that $\mathfrak{N}=0$. Then $\mathfrak{A}$ is not a nilalgebra and so must contain an idempotent. It follows that $\mathfrak{A}$ contains a principal idempotent $e$ and that $\mathfrak{A}=\mathfrak{A}_{e}(1) \oplus \mathfrak{A}_{e}(0)$. But then $\mathfrak{A}_{e}(0)$ is a nilideal of $\mathfrak{A}$ and so is zero, $e$ is the unity element of $\mathfrak{A}$. Write $e=u+v$ where $u$ and $v$ are pairwise orthogonal idempotents and have $\mathfrak{A}=\mathfrak{A}_{u}(1)$ $\oplus \mathfrak{A}_{u}(0)$ where $v$ is in $\mathfrak{A}_{u}(0)$. Then every $a$ of $\mathfrak{A}$ has the form $a=b+c$ where $b$ is in $\mathfrak{A}_{u}(1)$ and $c$ is in $\mathfrak{A}_{u}(0), e a=a=(u+v)(b+c)=b+v c=b+c, v c=c$. It is then 
clear that $\mathfrak{A}_{u}(0)=\mathfrak{A}_{v}(1)$. We now decompose $e$ as a sum $e=e_{1}+\cdots+e_{t}$ of pairwise orthogonal primitive idempotents $e_{i}$ and have a corresponding decomposition of $\mathfrak{A}$ as the direct sum $\mathfrak{A}=\mathfrak{A}_{1} \oplus \cdots \oplus \mathfrak{A}_{t}$ where $e_{i}$ is the unity element of $\mathfrak{A}_{i}$. Every nilideal of $\mathfrak{A}_{i}$ is a nilideal of $\mathfrak{A}$ and so $\mathfrak{A}_{i}$ must be semisimple. Moreover a decomposition $\mathfrak{A}_{i}=\mathfrak{B}_{i} \oplus \mathfrak{E}_{i}$ of $\mathfrak{A}_{i}$ results in a decomposition $e_{i}=u_{i}+v_{i}$ of $e_{i}$ contrary to our hypothesis that $e_{i}$ is primitive. Hence $\mathfrak{A}_{i}$ must not have a decomposition as a direct sum. Actually $\mathfrak{A}_{i}$ must have no ideals other than zero and $\mathfrak{A}_{i}$ since it can have no nilideals and any other nonzero proper ideal of $\mathfrak{A}$ would contain an idempotent $u \neq e$. We may now write $\mathfrak{A}_{i}$ as a central simple algebra over its center $\mathfrak{Z}_{i}$. Extend $\mathfrak{Z}_{i}$ to be an algebraically closed field $\Omega_{i}$ and designate the resulting simple algebra by $\mathfrak{B}_{i}$. Then $\mathfrak{B}_{i}$ is not a nilalgebra and if $x$ is in $\mathfrak{B}_{i}$, then the only idempotent in $\mathfrak{F}[x]$ can be $e_{i}$ since otherwise $\mathfrak{B}_{i}$ would be reducible. It follows that every element of $\mathfrak{B}_{i}$ has the form $\mathfrak{x}=\alpha e_{i}+f$ where $f$ is nilpotent; $\alpha$ is in $\Re_{i}$. As in the case of Jordan algebras the trace criterion implies that the set of all nilpotent elements of $\mathfrak{B}_{i}$ is a nilideal. Then $x=\alpha e_{i}, \mathfrak{B}_{i}$ has order one over $\mathfrak{\Omega}_{i}, \mathfrak{A}_{i}=\mathfrak{B}_{i}$ and we have proved

THEOREM 7. Every semi-simple static algebra is a commutative associative semi-simple algebra.

\section{Chapter IV. Standard algebras}

1. Flexible Jordan-admissible algebras. The theory of Jordan algebras is so much like the theory of associative algebras that it is natural to conjecture that both classes of algebras are members of a more general class with a similar theory. We shall present such a class of algebras here.

The class of algebras desired will satisfy the Jordan postulate $(x y) x^{2}$ $=x\left(y x^{2}\right)$ and the postulate of commutativity will be replaced by the postulate of flexibility. However, the formulation will be much more delicate since the structure of Jordan algebras is based upon formulas derived from the assumption $x\left(y x^{2}\right)=(x y) x^{2}$ and using the commutative law.

Let us first derive some consequences of the assumption of flexibility, that is, of the property that

$$
R_{x} L_{x}=L_{x} R_{x}
$$

for every $x$ of the algebra $\mathfrak{A}$. Then $R_{x+y} L_{x+y}-L_{x+y} R_{x+y}=\left(R_{x} L_{x}-L_{x} R_{x}\right)$ $+\left(R_{x} L_{y}-L_{y} R_{x}\right)+\left(R_{y} L_{x}-L_{x} R_{y}\right)+\left(R_{y} L_{y}-L_{y} R_{y}\right)=0$, and so

$$
R_{x} L_{y}-L_{y} R_{x}=L_{x} R_{y}-R_{y} L_{x}
$$

for every $x$ and $y$ of $\mathfrak{A}$. However formula (2) is equivalent to the equation $(x y) z+(z y) x=x(y z)+z(y x)$ of (1.29) and this latter equation may be written as

$$
L_{x y}-L_{y} L_{x}=R_{y x}-R_{y} R_{x}
$$


In particular we have

$$
L_{x x}-L_{x}^{2}=R_{x x}-R_{x}^{2}
$$

An algebra $\mathfrak{A}$ will be called a Jordan-admissible algebra if the algebra $\mathfrak{A}^{(+)}$ is a Jordan algebra. The multiplications $S_{x}$ of $\mathfrak{A}^{(+)}$are defined in terms of the multiplications of $\mathfrak{A}$ by $2 S_{x}=R_{x}+L_{x}$ and the product $x^{2}$ in $\mathfrak{A}$ coincides with this product in $\mathfrak{A}^{(+)}$. It follows that $\mathfrak{A}$ is Jordan-admissible if and only if

$$
\left(R_{x}+L_{x}\right)\left(R_{x x}+L_{x x}\right)=\left(R_{x x}+L_{x x}\right)\left(R_{x}+L_{x}\right)
$$

for every $x$ of $\mathfrak{A}$. Note that all Jordan algebras are Jordan-admissible since $\mathfrak{A}^{(+)}$and $\mathfrak{A}$ coincide for commutative algebras. Also every associative algebra is Jordan-admissible since $R_{x} L_{x}=L_{x} R_{x}$ and $R_{x x}=R_{x}^{2}, L_{x x}=L_{x}^{2}$ in an associative algebra.

If $x$ is any element of an algebra $\mathfrak{A}$ over a field $\mathfrak{F}$ there is a corresponding polynomial algebra $\mathfrak{A}_{x}=\mathfrak{F}\left[R_{x}, L_{x}, R_{x x}\right]$. When $\mathfrak{A}$ is flexible (4) implies that $\mathfrak{A}_{x}=\mathfrak{F}\left[R_{x}, L_{x}, L_{x x}\right]$. We now prove

TheOREM 1. Let $\mathfrak{A}$ be an algebra over a field $\mathfrak{F}$ of characteristic prime to 30. Then $\mathfrak{A}_{x}$ is a commutative algebra for every $x$ of $\mathfrak{A}$ if and only if $\mathfrak{A}$ is flexible and one of the relations

$$
\begin{array}{ll}
x\left(y x^{2}\right)=(x y) x^{2}, & \left(x^{2} y\right) x=x^{2}(y x), \\
(y x) x^{2}=\left(y x^{2}\right) x, & x^{2}(x y)=x\left(x^{2} y\right)
\end{array}
$$

holds for every $x$ and $y$ of $\mathfrak{A}$. Moreover if $\mathfrak{A}_{x}$ is commutative the algebra $\mathfrak{A}$ is Jordan-admissible and is power-associative, the algebra $\mathfrak{A}_{x}$ contains $R_{u}$ and $L_{u}$ for every power $u=x^{k}$ of $x$.

For the relations (6) are the commutativity relations

$$
\begin{array}{ll}
L_{x} R_{x x}=R_{x x} L_{x}, & R_{x} L_{x x}=L_{x x} R_{x}, \\
R_{x} R_{x x}=R_{x x} R_{x}, & L_{x} L_{x x}=L_{x x} L_{x} .
\end{array}
$$

If $\mathfrak{A}_{x}$ is commutative all of the relations (7) hold and (5) holds trivially. Moreover (1) holds and $\mathfrak{A}$ is a flexible Jordan-admissible algebra. Also $x(x x)=(x x) x, x\left(x x^{2}\right)=x^{2} x^{2}=\left(x^{2} x\right) x$ by (6) and the formula $x^{\alpha} x^{\beta}=x^{\alpha+\beta}$ holds for $\alpha+\beta=3,4$. Assume that this formula holds for $\alpha+\beta<n$ where $n>4$ and write $y=x^{n-3}$ in (6) to obtain $x x^{n-1}=x^{n-2} x^{2}, x^{n-2} x^{2}=x^{n-1} x$ so that $x x^{n-1}=x^{n-1} x$ and we may apply Lemma 1.2 to obtain the formula for $\alpha+\beta=n$. Hence $\mathfrak{A}$ is power-associative.

We now replace $x$ by $x+\lambda z$ in the first relation of (6) and equate the coefficients of $\lambda$ to obtain

$$
x[y(x z+z x)]+z\left(y x^{2}\right)=(x y)(x z+z x)+(z y) x^{2} .
$$

This formula may be linearized and results in a relation which may be 
written as

$$
\begin{aligned}
w[x(y z+z y)]+y[x(z w+w z)]+z[x(y w+w y)] \\
=(w x)(y z+z y)+(y x)(z w+w z)+(z x)(y w+w y) .
\end{aligned}
$$

This relation becomes

(10) $R_{x(y z+z y)}=R_{x} R_{y z+z y}+\left(R_{z}+L_{z}\right)\left(L_{y x}-L_{x} L_{y}\right)+\left(R_{y}+L_{y}\right)\left(L_{z x}-L_{x} L_{z}\right)$.

The assumption that $\mathfrak{A}_{x}$ contains $R_{u}$ and $L_{u}$ for $u=x^{k}$ and $k=1, \cdots, t$ imimplies that $R_{u}$ is in $\mathfrak{A}_{x}$ for $u=x^{t+1}$ when we replace $y$ by $x^{t-1}$ and put $z=x$ in (10). Then relation (3) implies that $L_{u}$ is in $\mathfrak{A}_{x}$.

Conversely let $\mathfrak{A}$ be flexible and let one of the relations (6) hold. By (2) with $y=x^{2}$ we see that

$$
R_{x} L_{x x}-L_{x x} R_{x}=L_{x} R_{x x}-R_{x x} L_{x}
$$

Hence the first two relations of (6) are equivalent when $\mathfrak{A}$ is flexible. The relation $R_{x} R_{x x}=R_{x x} R_{x}$ and (4) imply that $L_{x x}$ is commutative with $R_{x}$ while $L_{x} L_{x x}=L_{x x} L_{x}$ and (4) imply that $R_{x x} L_{x}=L_{x} R_{x x}$. Thus we see that the flexible law implies the equivalence of all of the relations (6) and all imply that $\mathfrak{A}_{\boldsymbol{x}}$ is a commutative algebra.

It should be noted that the relations (6) may be linearized and imply

$$
\begin{aligned}
{\left[R_{x}, L_{y z+z y}\right]+\left[R_{y}, L_{x z+z x}\right]+\left[R_{z}, L_{x y+y x}\right] } & =0, \\
{\left[R_{x}, R_{y z+z y}\right]+\left[R_{y}, R_{x z+z x}\right]+\left[R_{z}, R_{x y+y x}\right] } & =0, \\
{\left[L_{x}, L_{y z+z y}\right]+\left[L_{y}, L_{x z+z x}\right]+\left[L_{z}, L_{x y+y x}\right] } & =0,
\end{aligned}
$$

where, as usual, we mean $[S, T]=S T-T S$. The omitted relation is a trivial consequence of (12) and (2).

The relation (10) and the flexible law seem inadequate to yield a satisfactory theory and we shall strengthen both assumptions. It should be noted that all of the results above actually hold for rings.

2. Lie-admissible rings. Every ring $\mathfrak{A}$ determines an attached ring $\mathfrak{A}(-)$ which is the same additive group as $\mathfrak{A}$ but has a product $(x, y)$ defined in terms of the product $x y$ of $\mathfrak{A}$ by $(x, y)=x y-y x$. The right multiplications $T_{x}$ of $\mathfrak{A}^{(-)}$will then be defined in terms of the multiplications of $\mathfrak{A}$ by $T_{x}=R_{x}-L_{x}$.

We call $\mathfrak{A}$ a Lie-admissible ring if $\mathfrak{U}^{(-)}$is a Lie ring, that is, $T_{(x, y)}=T_{x} T_{y}$ $-T_{y} T_{x}$. Then $\mathfrak{A}$ is Lie-admissible if and only if

$$
R_{x y-y x}-L_{x y-y x}=\left(R_{x}-L_{x}\right)\left(R_{y}-L_{y}\right)-\left(R_{y}-L_{y}\right)\left(R_{x}-L_{x}\right) .
$$

Let us introduce the assumption that $\mathfrak{A}$ is also a flexible ring. We then use (3) to write $-R_{y x}+L_{x y}=-R_{y} R_{x}+L_{y} L_{x}, R_{x y}-L_{y x}=R_{x} R_{y}-L_{x} L_{y}$ and we add to obtain

$$
R_{x y-y x}+L_{x y-y x}=\left(R_{x} R_{y}-R_{y} R_{x}\right)-\left(L_{x} L_{y}-L_{y} L_{x}\right) .
$$


The right member of (15) is $\left(R_{x} R_{y}-R_{y} R_{x}\right)+\left(L_{x} L_{y}-L_{y} L_{x}\right)+\left(L_{y} R_{x}-R_{x} L_{y}\right)$ $+\left(R_{y} L_{x}-L_{x} R_{y}\right)$ and $R_{y} L_{x}-L_{x} R_{y}=L_{y} R_{x}-R_{x} L_{y}$ by (2). Then we may add (15) and (16) and remove the factor 2 to obtain

$$
R_{x y-y x}=R_{x}\left(R_{y}-L_{y}\right)-\left(R_{y}-L_{y}\right) R_{x} .
$$

We may also subtract (15) from (16) and obtain

$$
L_{x y-y x}=\left(L_{y}-R_{y}\right) L_{x}-L_{x}\left(L_{y}-R_{y}\right) .
$$

Conversely both (17) and (18) imply that $R_{x} L_{x}=L_{x} R_{x}$ so that $\mathfrak{A}$ is flexible and then (17) and (18) are equivalent to (15). We have proved

Theorem 2. A ring $\mathfrak{A}$ is a flexible Lie-admissible ring if and only if either (17) or (18) holds for every $x$ and $y$ of $\mathfrak{A}$.

A Jordan ring is trivially flexible and Lie-admissible since (18) is satisfied when $x y=y x, R_{y}=L_{y}$. It is well known that an associative algebra is Lie admissible and is, of course, flexible. However, we may prove

TheOREM 3. An alternative ring whose characteristic is prime to six is Lieadmissible if and only if it is associative.

For if $\mathfrak{A}$ is alternative then $\mathfrak{A}$ is flexible and $x(x y)=(x x) y, x(z y)+z(x y)$ $=(x z+z x) y$,

$$
R_{x y}=R_{x} R_{y}+\left(L_{x} R_{y}-R_{y} L_{x}\right) .
$$

But by (2) $L_{x} R_{y}-R_{y} L_{x}=R_{x} L_{y}-L_{y} R_{x}$ and so from $R_{y x}=R_{y} R_{x}+\left(L_{y} R_{x}-R_{x} L_{y}\right)$ we obtain $R_{x y-y x}=\left(R_{x} R_{y}-R_{y} R_{x}\right)-2\left(L_{y} R_{x}-R_{x} L_{y}\right)$. This combined with (17) yields $3\left(L_{y} R_{x}-R_{x} L_{y}\right)=0, L_{y} R_{x}=R_{x} L_{y}$ since the characteristic of $\mathfrak{A}$ is prime to six. Thus $(y z) x=y(z x)$ for every $x, y, z$ of $\mathfrak{A}$ and $\mathfrak{A}$ is associative.

3. Standard algebras. An algebra $\mathfrak{A}$ over a field $\mathfrak{F}$ will be called a standard algebra if (17) holds and if

$$
R_{x(y z)}=R_{x} R_{y z}+R_{y}\left(R_{x z}-R_{x} R_{z}\right)+R_{z}\left(R_{x y}-R_{x} R_{y}\right)
$$

for every $x, y, z$ of $\mathfrak{A}$. (20) is known to be a consequence of the defining assumptions for Jordan algebras and is a trivial consequence of the property $R_{x y}=R_{x} R_{y}$ of associative algebras. We note the following simple result

TheOREM 4. A Lie algebra $\mathfrak{A}$ is a standard algebra if and only if all products of four elements of $\mathfrak{A}$ are zero.

For when $\mathfrak{A}$ is a Lie algebra $R_{x(y z)}=R_{x} R_{y z}-R_{y z} R_{x}$. Then (20) is equivalent to $-R_{y z} R_{x}=\left(R_{z} R_{y}-R_{y} R_{z}\right) R_{x}=R_{y} R_{z} R_{x}+R_{z} R_{y} R_{x}$, that is, to $2 R_{y} R_{z} R_{x}=0$. Thus $[(w y) z] x=0$ for every $x, y, z$, w of $\mathfrak{A}, L_{(w y) z}=0=-R_{(w y) z}, R_{w y} R_{z}=R_{z} R_{w y}$, $R_{w y} R_{x}=0, R_{z} R_{w y}=0,(x z)(w y)=0$, all products of four elements of $\mathfrak{A}$ are zero. The converse clearly implies that $[(w y) z] x=0, R_{y} R_{z} R_{x}=0$ and so that (20) holds. 
We shall now derive some identities which are a consequence of the relation (20) and the flexible law. We first write (20) as

$$
w[x(y z)]+[(w y) x] z+[(w z) x] y=(w x)(y z)+(w y)(x z)+(w x)(x y)
$$

and then have $R_{y z} L_{w}+L_{w y} R_{z}+L_{w z} R_{y}=L_{w} R_{y z}+R_{z} L_{w y}+R_{y} L_{w z}$. However $R_{y z} L_{w}-L_{w} R_{y z}=L_{y z} R_{w}-R_{w} L_{y z}$ by (2) and we replace $w$ by $x$ to obtain

$$
R_{x} L_{y z}+R_{y} L_{x z}+R_{z} L_{x y}=L_{y z} R_{x}+L_{x z} R_{y}+L_{x y} R_{z} .
$$

Observe that if the characteristic of $\mathfrak{A}$ is prime to three then (22) implies that $R_{x} L_{x x}=L_{x x} R_{x}$ and so that every $\mathfrak{A}_{x}$ is commutative. We have proved

THEOREM 5. Let $\mathfrak{A}$ be a standard algebra over a field $\mathfrak{F}$ whose characteristic is prime to six. Then $\mathfrak{A}$ is a power-associative Jordan-admissible algebra and $\mathfrak{A}_{x}$ is commutative for every $x$ of $\mathfrak{A}$, that is, the transformations $R_{u}, L_{u}, R_{v}, L_{v}$ commute for all powers $u$ and $v$ of $x$.

The identity (21) may be rewritten with $w$ and $z$ interchanged and implies that $L_{(z y) x}+L_{y} L_{x} L_{z}+L_{z} R_{x} R_{y}=L_{y} L_{z x}+L_{x} L_{z y}+L_{z} R_{x y}$. However $R_{x y}-R_{x} R_{y}$ $=L_{y x}-L_{x} L_{y}$ and so we have

$$
L_{(z y) x}=L_{x} L_{z y}+L_{y}\left(L_{z x}-L_{x} L_{z}\right)+L_{z}\left(L_{y x}-L_{x} L_{y}\right) .
$$

We note the consequences

$$
R_{x(x x)}=3 R_{x} R_{x x}-2 R_{x}^{3}
$$

and

$$
L_{(x x) x}=3 L_{x} L_{x x}-2 L_{x}^{3}
$$

of (20) and (23) respectively.

4. Solvable algebras. Our first major result will involve a subalgebra $\mathfrak{B}$ of a standard algebra $\mathfrak{A}$. Define $\mathfrak{B}_{0}$ to be the vector space of all mappings on $\mathfrak{A}$ of the form $R_{x}+L_{y}$ for $x$ and $y$ in $\mathscr{B}$ and $\mathfrak{B}^{*}$ to be the associative algebra of all finite sums of products of elements of $\mathfrak{B}_{0}$. Then we may prove

THEOREM 6. Let $\mathfrak{B}$ be a solvable subalgebra of a standard algebra $\mathfrak{A}$ over $\mathfrak{F}$ of characteristic prime to six. Then $\mathfrak{B}^{*}$ is nilpotent.

The result is true for $\mathfrak{B}$ of order one since then $\mathfrak{B}=x \mathfrak{F}, x^{2}=0, R_{x}^{3}=0$ by (24), $L_{x}^{3}=0$ by (25), $\mathfrak{B}^{*}$ is the commutative algebra $\mathfrak{F}\left[R_{x}, L_{x}\right]$ and is clearly nilpotent. Assume the result true for algebras of order $m-1$ and let $\mathfrak{B}$ have order $m$. Every solvable algebra $\mathfrak{B}$ has the form $\mathfrak{B}=\mathfrak{C}+w \mathfrak{F}$ where $\mathfrak{B} \mathfrak{B} \leqq \mathfrak{S}$ and $\sqrt{5}$ has order $m-1$ and so $\mathfrak{C}^{*}$ is nilpotent. We propose to show that $\mathfrak{B}_{0}^{3} \leqq \mathfrak{S}=\mathfrak{B}^{*} \mathfrak{C}^{*}+\mathfrak{C}^{*}$.

Since $\mathfrak{B}_{0}$ is a vector space it is sufficient to prove that all products $S_{x} S_{y} S_{z}$ are in $\mathfrak{S}$ where $S_{x}$ is the symbol for a multiplication and $x, y, z$ are either in 
$\sqrt{5}$ or equal to $w$. Now (20) and $\mathfrak{B B} \leqq \mathbb{C}$ imply that

$$
H_{1}(x, y, z)=R_{x} R_{y} R_{z}+R_{z} R_{y} R_{x}
$$

is in $\mathfrak{S}$ for every $x, y, z$ of $\mathfrak{B}$. Then $R_{x} R_{y} R_{z}$ is in $\mathfrak{S}$ if $z$ is in $\mathfrak{E}, R_{x} R_{y} R_{w}$ $=H_{1}(x, y, z)-R_{w} R_{y} R_{x}$ is in $\mathfrak{S}$ if $x$ is in $\left(5,2 R_{w} R_{y} R_{w}\right.$ is in (5 for every $y$. Thus $R_{x} R_{y} R_{z}$ is in $\mathfrak{S}$ for every $x, y, z$ of $\mathfrak{B}$. Similarly (23) implies that every $L_{x} L_{y} L_{z}$ is in $\mathfrak{S}$. But $R_{x} L_{y} L_{z}=R_{x}\left(L_{z y}-R_{y x}+R_{y} R_{z}\right)$ is then in $\mathscr{A}$ and so is $L_{x} R_{y} R_{z}=L_{x}\left(R_{y z}\right.$ $\left.-L_{z y}-L_{y} L_{z}\right)$. It remains to consider products $R_{x} L_{y} R_{z}, L_{x} R_{y} L_{z}, R_{x} R_{y} L_{z}$, $L_{x} L_{y} R_{z}$.

We note next that the assumption (17) implies that $R_{z} R_{x y-y x}=R_{z}\left(R_{x} R_{y}\right.$ $\left.-R_{y} R_{x}\right)+R_{z}\left(L_{y} R_{x}-R_{x} L_{y}\right)$ and so $H=R_{z}\left(L_{y} R_{x}-R_{x} L_{y}\right)$ is in $\mathfrak{S}$. If $x$ is in then $R_{z} L_{y} R_{x}$ is in $\mathfrak{S}$. Hence let $x=w$. If $y$ is in (5) then $R_{z} L_{y} R_{x}=R_{z} R_{x} L_{y}+H$ is in $\mathfrak{S}$. It then remains to consider $R_{z} L_{w} R_{w}=\left(L_{z} R_{w}+L_{w} R_{z}-R_{w} L_{z}\right) R_{w}$ (by formula (21)) which is in $\mathfrak{S}$ if and only if $R_{w} L_{z} R_{w}$ is in $\mathfrak{S}$. We have already seen that $R_{w} L_{z} R_{w}$ is in $\mathfrak{S}$ if $z$ is in $\mathbb{S}$ so there remains the case of a product $R_{w} L_{w} R_{w}=L_{w} R_{w} R_{w}$ which is in $\mathfrak{S}$. It follows that all products $R_{z} L_{y} R_{x}$ and all products $R_{z} R_{x} L_{y}$ are in $\mathfrak{S}$. We also have $L_{z} R_{x y-y x}=L_{z}\left(R_{x} R_{y}-R_{y} R_{x}\right)$ $+L_{z}\left(L_{y} R_{x}-R_{x} L_{y}\right)$ and so $L_{z} L_{y} R_{x}-L_{z} R_{x} L_{y}$ is in $\mathfrak{W}$. The remainder of the proof is exactly as above.

We have now shown that $\mathfrak{B}_{0}^{3} \leqq \mathfrak{B}^{*} \mathfrak{C}^{*}+\mathfrak{C}^{*}$ so that $\mathfrak{B}^{* 4} \leqq \mathfrak{B}^{*} \mathfrak{C}^{*}, \mathfrak{B}^{* 3 k+1}$ $\leqq \mathfrak{B}^{*} \mathfrak{C}^{* k}=0$ for some $k, \mathfrak{B}^{*}$ is nilpotent.

5. Nilalgebras. The procedure we shall follow in the study of standard nilalgebras is an extension to noncommutative algebras of that used for Jordan algebras. We observe first that if $\mathfrak{B}$ is a subalgebra of $\mathfrak{A}$ then $x \mathfrak{B}^{*} \leqq \mathfrak{B}$ if and only if $\mathfrak{B}$ contains both $x b$ and $b x$ for every $b$ of $\mathfrak{B}$. There always exists an idempotent linear transformation $E$ on $\mathfrak{A}$ such that $\mathfrak{B}=\mathfrak{A} E$ and we define $\mathfrak{W}$ to be the set of all linear transformations $T$ on $\mathfrak{A}$ such that $E T=E T E$. Then $\mathfrak{W}$ is an associative algebra and we have

LEMMA 1. If $x$ is in $\mathfrak{A}$ then $x \mathfrak{B}^{*} \leqq \mathfrak{B}$ if and only if $R_{x}$ and $L_{x}$ are in $\mathfrak{W}$.

For the generic element of $\mathfrak{B}$ is $b=a E$ where $a$ is the generic element of $\mathfrak{A}$. Then an element $y$ is in $\mathfrak{X} E$ if and only if $y=y E$ and so $x b=x(a E)=a\left(E L_{x}\right)$ and $b x=(a E) x=a\left(E R_{x}\right)$ are in $\mathfrak{B}$ if and only if $E L_{x}=E L_{x} E, E R_{x}=E R_{x} E$, that is, $R_{x}$ and $L_{x}$ are in $\mathfrak{W}$.

Note that $b \mathfrak{B}^{*} \leqq \mathfrak{B}$ for every $b$ of $\mathfrak{B}$ and so $R_{b}$ and $L_{b}$ are in $\mathfrak{W}$. We next prove

LEMMA 2. Let $\mathfrak{B}$ be a subalgebra of a power-associative algebra $\mathfrak{A}$ and let $R_{x}, L_{x}, R_{x x}$ be in $\mathfrak{W}$ for $x$ in $\mathfrak{A}$. Then $\mathfrak{B}$ is an ideal of the algebra $\mathfrak{B}[x]$ of all polynomials in $x$ with coefficients in either $\mathfrak{B}$ or $\mathfrak{F}$.

For Lemma 1.5 implies that the algebra $\mathfrak{A}_{x}$ of all polynomials in $R_{x}, L_{x}, R_{x x}$ contains $R_{u}$ and $L_{u}$ for all powers $u=x^{k}$ of $x$. Then $\mathfrak{A}_{x} \leqq \mathfrak{W}$ and every $R_{u}$ is in $\mathfrak{W}, u \mathfrak{B}^{*} \leqq \mathfrak{B}$. It follows that $\mathfrak{B}[x]=\mathfrak{F}[x]+\mathfrak{B}$ and that $\mathfrak{B}$ is an ideal of $\mathfrak{B}[x]$. 
The assumption that $\mathfrak{A}$ is a standard algebra will now be introduced and we shall prove

Lemma 3. Let $R_{x}$ and $L_{x}$ be in $W$ and $y=b x^{2}$ where $b$ is in $\mathfrak{B}$. Then $R_{y}$ and $L_{y}$ are in $\mathfrak{W}$.

We first apply (20), (13), (23), (14), (4) to obtain the relations

$$
\begin{aligned}
& R_{x(b x)}=R_{x} R_{b x}+R_{b}\left(R_{x x}-R_{x}^{2}\right)+R_{x}\left(R_{x b}-R_{x} R_{b}\right), \\
& R_{b} R_{x x}+R_{x} R_{b x+x b}=R_{x x} R_{b}+R_{b x+x b} R_{x}, \\
& L_{(x b) x}=L_{x} L_{x b}+L_{b}\left(L_{x x}-L_{x}^{2}\right)+L_{x}\left(L_{b x}-L_{x} L_{b}\right), \\
& L_{b} L_{x x}+L_{x} L_{x b+b x}=L_{x b+b x} L_{x}+L_{x x} L_{b}, \\
& L_{b} L_{x x}=L_{b}\left(R_{x x}+L_{x}^{2}-R_{x}^{2}\right), \quad L_{x x} L_{b}=\left(R_{x x}+L_{x}^{2}-R_{x}^{2}\right) L_{b} .
\end{aligned}
$$

Since $b x$ and $x b$ are in $\mathscr{B}$ we see from (27) that $R_{b} R_{x x}$ is in $\mathfrak{W}$, from (28) that $R_{x x} R_{b}$ is in $\mathfrak{W}$, from (29) that $L_{b} L_{x x}$ is in $\mathfrak{W}$, from (30) that $L_{x x} L_{b}$ is in $\mathfrak{W}$, and from (31) that $L_{b} R_{x x}$ and $R_{x x} L_{b}$ are in $\mathfrak{W}$. Now by (20) and (17) respectively,

$$
\begin{aligned}
& R_{b(x x)}=R_{b} R_{x x}+2 R_{x}\left(R_{b x}-R_{b} R_{x}\right), \\
& R_{(x x) b}=R_{b(x x)}+R_{x x}\left(R_{b}-L_{b}\right)-\left(R_{b}-L_{b}\right) R_{x x}
\end{aligned}
$$

so that both $R_{y}$ and $R_{(x x) b}$ are in $\mathfrak{W}$. But by (3),

$$
R_{(x x) b}-R_{x x} R_{b}=L_{b(x x)}-L_{x x} L_{b}
$$

and so $L_{y}$ is also in $\mathfrak{W}$ as desired.

We write $u=x x^{2}$ and use (24) together with Theorem 5 to write $R_{b} R_{u}$ $=3 R_{b} R_{x x} R_{x}-2 R_{b} R_{x}^{3}, R_{u} R_{b}=3 R_{x} R_{x x} R_{b}-2 R_{x}^{3} R_{b}$. Since $R_{x x} R_{b}$ and $R_{b} R_{x x}$ are in $\mathfrak{B}$, we see that $R_{u} R_{b}$ and $R_{b} R_{u}$ are in $\mathfrak{B}$ for every $b$ of $\mathfrak{B}$. Similarly, $R_{u} L_{b}$ and $L_{b} R_{u}$ are in $\mathfrak{W}$. But by (20) we have

$$
R_{b u}=R_{b} R_{u}+R_{x x}\left(R_{b x}-R_{b} R_{x}\right)+R_{x}\left(R_{y}-R_{b} R_{x x}\right)
$$

which implies that $R_{b u}$ is in $\mathfrak{W}$, and by (17),

$$
R_{u b}=R_{b u}+R_{u}\left(R_{b}-L_{b}\right)-\left(R_{b}-L_{b}\right) R_{u}
$$

and then $R_{u b}$ is in $\mathfrak{W}$. But

$$
R_{u(b x)}=R_{u} R_{b x}+R_{b}\left(R_{v}-R_{u} R_{x}\right)+R_{x}\left(R_{u b}-R_{u} R_{b}\right)
$$

where $v=u x=x^{4}, b x$ is in $\mathfrak{B}$ and so $R_{u(b x)}$ is in $\mathfrak{W}$. This shows that $R_{b} R_{v}$ is in $\mathfrak{B}$. However $R_{v}=R_{x u}$ and the same computation used in the commutative Jordan case gives

$$
R_{v}=R_{x x}^{2}+4 R_{x x} R_{x}^{2}-4 R_{x}^{4}
$$

and so $R_{b} R_{x x}^{2}$ is in $\mathfrak{W}$. We now use (28) to form 


$$
R_{x x} R_{b} R_{x x}=-R_{x}\left(R_{x x} R_{b x+x b}\right)+R_{x x}^{2} R_{b}+R_{x x} R_{b x+x b} R_{x}
$$

by multiplication on the left and

$$
R_{x x} R_{b} R_{x x}=R_{b} R_{x x}^{2}+R_{x} R_{b x+x b} R_{x x}-\left(R_{b x+x b} R_{x x}\right) R_{x} .
$$

Then we subtract to see that $R_{x x}^{2} R_{b}$ is in $\mathfrak{Y S}$.

We now write $y^{2}=y\left(b x^{2}\right)$ and form

$$
R_{y y}=R_{y}^{2}+R_{b}\left(R_{y(x x)}-R_{y} R_{x x}\right)+R_{x x}\left(R_{y b}-R_{y} R_{b}\right) .
$$

We then use $R_{y(x x)}-R_{y} R_{x x}=2 R_{x}\left(R_{y x}-R_{y} R_{x}\right)$ as well as

$$
\begin{aligned}
& R_{x y}=R_{x} R_{y}+R_{b}\left(R_{u}-R_{x} R_{x x}\right)+R_{x x}\left(R_{x b}-R_{x} R_{b}\right), \\
& R_{y x}=R_{x y}+R_{y}\left(R_{x}-L_{x}\right)-\left(R_{x}-L_{x}\right) R_{y}
\end{aligned}
$$

to see that $R_{x y}$ and $R_{y x}$ are in $\mathfrak{W}, R_{b}\left(R_{y(x x)}-R_{y} R_{x x}\right)$ is in $\mathfrak{W}$. The term $R_{x x} R_{y} R_{b}=R_{x x}\left[R_{b} R_{x x}+2 R_{x}\left(R_{b x}-R_{b} R_{x}\right)\right] R_{b}=\left(R_{x x} R_{b}\right)^{2}+2 R_{x}\left(R_{x x} R_{b x} R_{b}\right.$ $\left.-R_{x x} R_{b} R_{x} R_{b}\right)$ is in $\mathfrak{B}$ and we finally compute

$$
R_{b y}=R_{b} R_{y}+R_{x x}\left(R_{b b}-R_{b}^{2}\right)+R_{b}\left(R_{y}-R_{b} R_{x^{2}}\right)
$$

and

$$
R_{y b}=R_{b y}+R_{y}\left(R_{b}-L_{b}\right)+\left(R_{b}-L_{b}\right) R_{y} .
$$

The product $\left[R_{x x}\left(R_{b}-L_{b}\right)\right] R_{y}$ is in $\mathfrak{W}$, the product $R_{x x} R_{y}\left(R_{b}-L_{b}\right)$ $=R_{x x} R_{b} R_{x x}\left(R_{b}-L_{b}\right)+2 R_{x} R_{x x}\left(R_{b x}-R_{b} R_{x}\right)\left(R_{b}-L_{b}\right)$ is in $\mathfrak{W}$, and the product $R_{x x} R_{b y}=\left(R_{x x} R_{b}\right) R_{y}+R_{x x}^{2}\left(R_{b b}-R_{b}^{2}\right)+\left(R_{x x} R_{b}\right)\left(R_{y}-R_{b} R_{x x}\right)$ is in $\mathfrak{Y}$. But then $R_{x x} R_{y b}$ is in $\mathfrak{B}$ and we have proved that $R_{y y}$ is in $\mathfrak{W}$, which completes the proof of

LeMma 4. Let $R_{x}$ and $L_{x}$ be in $\mathfrak{W}, y=b x^{2}$ where $b$ is in $\mathfrak{B}$. Then $\mathfrak{A}_{y}$ is in $\mathfrak{W}$.

We are now ready to prove

Theorem 7. Let $\mathfrak{A}$ be a standard nilalgebra. Then $A$ is a solvable algebra.

The result is trivial for algebras of order one and so we assume it true for algebras of lower order than that of $\mathfrak{A}$. Also the result is true if $\mathfrak{A}$ consists of the associative algebra $\mathfrak{F}[x]$ generated by an element $x$ of $\mathfrak{A}$. Hence assume that $\mathfrak{A} \neq \mathfrak{F}[x]$ and thus that $\mathfrak{A}$ contains a proper subalgebra. Let $\mathfrak{B}$ be a maximal proper subalgebra of $\mathfrak{A}$. Then by the hypothesis of the induction, $\mathfrak{B}$ is solvable, $\mathfrak{B}^{*}$ is nilpotent, $\mathfrak{A} \mathfrak{B}^{* k}=0$. Let $t$ be the least integer such that $\mathfrak{Y P}^{* t} \leqq \mathfrak{B}$. Then clearly $t \geqq 1$ and there exists an element $z$ of $\mathfrak{A}$ such that $z \mathfrak{B}^{* t} \leqq \mathfrak{B}, z^{\mathfrak{B}^{* t-1}}$ is not $\leqq \mathfrak{B}$. Thus there is an element $x$ in $z \mathfrak{B}^{* t-1}$ but not in $\mathfrak{B}$ and $x \mathfrak{B}^{*} \leqq \mathfrak{B}$. By Lemmas 1 and 4 , if $b$ is in $\mathfrak{B}$, then $y \mathfrak{B}^{*} \leqq \mathfrak{B}, y^{2} \mathfrak{B}^{*} \leqq \mathfrak{B}$ for $y=b x^{2}$. Thus we have either some such $y$ not in $\mathfrak{B}$ or $\mathfrak{B} x^{2} \leqq \mathfrak{B}$. In either case we 
have shown the existence of an element $w$ not in $\mathscr{B}$ such that $w \mathfrak{B}^{*} \leqq \mathfrak{B}, \mathfrak{B} w^{2}$ $\leqq \mathfrak{B}$. This latter condition implies $R_{w^{2}}$ in $\mathfrak{W}$ as the proof of Lemma 1 shows and so the hypotheses of Lemma 2 are satisfied. Since $x$ is not in $\mathfrak{B}, \mathfrak{B}[x]=\mathfrak{A}$ by the maximality of $\mathfrak{A}$ and by Lemma $2, \mathfrak{B}$ is a solvable ideal of $\mathfrak{A}$. The nilalgebra $\mathfrak{A}-\mathfrak{B}$ is solvable by the induction hypothesis and so $\mathfrak{A}$ is solvable.

6. Trace admissibility of standard algebras. Let $\mathfrak{A}$ be a standard algebra and define its trace function $\tau(x, y)$ to be the trace of the linear transformation $R_{x y}$. By (17) we have $\tau(x, y)=\tau(y, x)$. Also $R_{x(y z)}-R_{z(x y)}=R_{x} R_{y z}+R_{y}\left(R_{x z}-R_{x} R_{z}\right)$ $+R_{z}\left(R_{x y}-R_{x} R_{y}\right)-R_{z} R_{x y}-R_{y}\left(R_{z x}-R_{z} R_{x}\right)-R_{x}\left(R_{z y}-R_{z} R_{y}\right)=R_{x} R_{y z-z y}$ $+R_{y} R_{x z-z x}+R_{y}\left(R_{z} R_{x}\right)-\left(R_{z} R_{x}\right) R_{y}+\left(R_{x} R_{z}\right) R_{y}-R_{y}\left(R_{x} R_{z}\right)$. However $R_{x} R_{y z-z}$ $+R_{y} R_{x z-z x}=R_{x}\left[R_{y}\left(R_{z}-L_{z}\right)-\left(R_{z}-L_{z}\right) R_{y}\right]+R_{y}\left[R_{x}\left(R_{z}-L_{z}\right)-\left(R_{z}-L_{z}\right) R_{x}\right]$ $=R_{x}\left[R_{y}\left(R_{z}-L_{z}\right)\right]-\left[R_{y}\left(R_{z}-L_{z}\right)\right] R_{x}+R_{y}\left[R_{x}\left(R_{z}-L_{z}\right)\right]-\left[R_{x}\left(R_{z}-L_{z}\right)\right] R_{y}$. Then $\tau(x, y z)-\tau(z, x y)=0, \tau(x, y z)=\tau(x y, z)$. If $x y$ is nilpotent it generates a solvable algebra, $R_{x y}$ is nilpotent, $\tau(x, y)=0$. If $e$ is an idempotent $\tau(e, e)$ is the trace of $R_{e}$ and is not zero. We have proved that $\tau(x, y)$ is an admissible trace function for $\mathfrak{A}$.

However, we may now show that $\tau(x, y)$ is an admissible trace function for $\mathfrak{A}^{(+)}$. For $\tau(x, y)=\tau(y, x), \tau(x \cdot y, z)=\tau(x y+y x, z) / 2=[\tau(x y, z)+\tau(y x, z)] / 2$ $=(\tau x, y z) / 2+\tau\left(y, x_{z}\right) / 2, \tau(y, x z)=\tau(x z, y)=\tau(x, z y), \tau(x \cdot y, z)=[\tau(x, y z)$ $+\tau(x, z y)] / 2=\tau(x, y \cdot z)$. If $x \cdot y$ is nilpotent so is $x y+y x, R_{x y+y x}$ has zero trace, $\tau(x, y)+\tau(y, x)=2 \tau(x, y)=0, \tau(x, y)=0$. If $e$ is idempotent $\tau(e, e) \neq 0$ as was shown above.

It follows now that the set of all elements $x$ such that $\tau(x, y)=0$ for every $\boldsymbol{y}$ of $\mathfrak{A}$ is the radical of both $\mathfrak{A}$ and $\mathfrak{U}^{(+)}$. We have proved

THeORem 8. Let $\mathfrak{A}$ be a standard algebra over, a nonmodular field. Then $\mathfrak{A}$ is trace-admissible, there is a trace function which is admissible both for $\mathfrak{A}$ and for $\mathfrak{A}^{(+)}$, the radical of $A$ coincides with the radical of $\mathfrak{X}^{(+)}, \mathfrak{A}$ is semi-simple if and only if $\mathfrak{A}^{(+)}$is semi-simple.

\section{Chapter V. Quasiassociative algebras}

1. The algebra $\mathfrak{A}(\lambda)$. If $\mathfrak{A}$ is any algebra over a field $\mathfrak{F}$ of characteristic not two and $\lambda$ is in $\mathfrak{F}$ we define $\mathfrak{A}(\lambda)$ to be that algebra which is the same vector space over $\mathfrak{F}$ as $\mathfrak{A}$ but whose product $x \cdot y$ is defined in terms of the product $x y$ of $\mathfrak{A}$ by $x \cdot y=\lambda x y+(1-\lambda) y x$. Then $\mathfrak{A}(1)=\mathfrak{A}, \mathfrak{A}(0)$ is antiisomorphic to $\mathfrak{A}, \mathfrak{A}(1 / 2)=\mathfrak{A}^{(+)}$.

Theorem 1. If $\mathfrak{A}$ is power-associative so is $\mathfrak{A}(\lambda)$ for every $\lambda$ of $\mathfrak{F}$ and indeed powers in $\mathfrak{A}(\lambda)$ coincide with powers in $\mathfrak{A}$. Also $\mathfrak{A}(\lambda)$ is flexible for every $\lambda$ if and only if $\mathfrak{A}$ is flexible.

For when $\mathfrak{A}$ is power-associative the power $x^{n}$ of every $x$ of $\mathfrak{A}$ is uniquely defined in $\mathfrak{A}$ for every positive integer $n$. Suppose that $x^{t}$ is the same in $\mathfrak{A}(\lambda)$ as in $\mathfrak{A}$ for every $t<n$, a result true for $t=1$. Then $x^{r} \cdot x^{n-r}=\lambda x^{r} x^{n-r}$ $+(1-\lambda) x^{n-r} x^{r}=\lambda x^{n}+(1-\lambda) x^{n}=x^{n}$ for every $r=1, \cdots, n-1$. It follows 
that $n$th powers of $x$ in $\mathfrak{A}(\lambda)$ all coincide with $n$th powers in $\mathfrak{A}$ and $\mathfrak{A}(\lambda)$ is power-associative. If $\mathfrak{A}(\lambda)$ is flexible for every $\lambda$ then in particular $\mathfrak{A}(1)=\mathfrak{A}$ is flexible. Conversely if $\mathfrak{A}$ is flexible then $(x \cdot y) \cdot x=\lambda[\lambda x y+(1-\lambda) y x]$ $\cdot x+(1-\lambda) x[\lambda x y+(1-\lambda) y x]=\lambda^{2}(x y) x+(1-\lambda)^{2} x(y x)+\lambda(1-\lambda)[(y x) x+x(x y)]$ and $x \cdot(y \cdot x)=\lambda x[\lambda y x+(1-\lambda) x y]+(1-\lambda)[\lambda y x+(1-\lambda) x y] x=\lambda^{2} x(y x)+(1-\lambda)^{2}$ $\cdot(x y) x+\lambda(1-\lambda)[x(x y)+(y x) x]$. The flexibility of $\mathfrak{A}$ implies that $\lambda^{2}(x y) x$ $+(1-\lambda)^{2} x(y x)=\lambda^{2} x(y x)+(1-\lambda)^{2}(x y) x$ and so $x \cdot(y \cdot x)=(x \cdot y) \cdot x, A(\lambda)$ is flexible.

Theorem 2. An algebra $\mathfrak{A}$ is Jordan-admissible if and only if $\mathfrak{X}(\lambda)$ is Jordanadmissible for every $\lambda$. Indeed $\mathfrak{A}^{(+)}=[\mathfrak{A}(\lambda)]^{(+)}$for every $\lambda$.

For $[\mathfrak{A}(\lambda)]^{(+)}$is defined relative to the product $(x \cdot y+y \cdot x) / 2=[\lambda x y$ $+(1-\lambda) y x+\lambda y x+(1-\lambda) x y] / 2=(x y+y x) / 2$.

In a similar fashion we may study $[\mathfrak{H}(\lambda)]^{(-)}$. This algebra is defined relative to the product $(x, y)=x \cdot y-y \cdot x=\lambda x y+(1-\lambda) y x-\lambda y x-(1-\lambda) x y$ $=(2 \lambda-1)(x y-y x)$. If $\lambda=1 / 2$ then $\mathfrak{A}(\lambda)$ is the commutative algebra $\mathfrak{H}^{(+)}$, $[\mathfrak{A}(\lambda)]^{(-)}$is a zero algebra and is always Lie-admissible. Otherwise $(x, y)=x T_{y}$ where $T_{y}=(2 \lambda-1) S_{y}$ and $S_{y}$ is the generic right multiplication for the algebra $\mathfrak{A}^{(-)}$. The algebra $\mathfrak{A}$ is Lie-admissible if and only if $S_{[x, y]}=S_{x} S_{y}-S_{y} S_{x}$ where $[x, y]=x y-y x=x S_{y}$ and if $\alpha=2 \lambda-1 \neq 0$ we see that $T_{(x, y)}=\alpha T_{[x, y]}$ $=\alpha^{2} S_{[x, y]}=\alpha^{2}\left(S_{x} S_{y}-S_{y} S_{x}\right)=T_{x} T_{y}-T_{y} T_{x}$. Conversely if $T_{(x, y)}=T_{x} T_{y}-T_{y} T_{x}$ then $S_{[x, y]}=S_{x} S_{y}-S_{y} S_{x}$ and we have proved

Theorem 3. An algebra $\mathfrak{A}$ is Lie-admissible if and only if $\mathfrak{A}(\lambda)$ is Lieadmissible for every $\lambda$ of $\mathfrak{F}$.

Let us henceforth restrict our attention to the case of algebras $\mathfrak{A}(\lambda)$ defined for $\lambda \neq 1 / 2$. Then $x \cdot y-y \cdot x=(2 \lambda-1)(x y-y x), x \cdot y+y \cdot x=x y+y x$ and so $2 x y=(2 \lambda-1)^{-1}[x \cdot y-y \cdot x+(2 \lambda-1)(x \cdot y+y \cdot x)]=(2 \lambda-1)^{-1} 2[\lambda x \cdot y$ $+(\lambda-1) y \cdot x]$. Since $1-(2 \lambda-1)^{-1}(\lambda-1)=(2 \lambda-1)^{-1}[2 \lambda-1-(\lambda-1)]$ $=\lambda(2 \lambda-1)^{-1}$, we have proved

Theorem 4. Let $\lambda \neq 1 / 2$ and $\mathfrak{S}=\mathfrak{A}(\lambda)$. Then $\mathfrak{A}=\mathfrak{S}(\mu)$ where $\mu=(2 \lambda-1)^{-1} \lambda$.

Theorem 4 implies that the ideals of $\mathfrak{A}$ and of $\mathfrak{S}=\mathfrak{A}(\lambda)$ coincide for every $\lambda \neq 1 / 2$. Indeed if $\mathfrak{B}$ is a subspace of $\mathfrak{A}$ such that $b y$ and $y b$ are in $\mathfrak{B}$ for every $b$ of $\mathfrak{B}$ and $y$ of $\mathfrak{A}$ it is true that $b \cdot y$ and $y \cdot b$ are in $\mathfrak{B}$ and conversely. When $\mathfrak{A}$ is a direct sum $\mathfrak{A}=\mathfrak{B} \oplus \mathbb{C}$ then $b c=c b=0$ for every $b$ of $\mathfrak{B}$ and $c$ of $\mathfrak{E}$, $b \cdot c=c \cdot b=0, \mathfrak{Y}(\lambda)=\mathfrak{B}(\lambda) \oplus \mathfrak{C}(\lambda)$. When $\mathfrak{B}$ is solvable so is $\mathfrak{B}(\lambda)$ and when $\mathfrak{B}$ is strongly nilpotent so is $\mathscr{B}(\lambda)$. Evidently the maximal solvable ideal of $\mathfrak{A}$ coincides with the maximal solvable ideal of $\mathfrak{A}(\lambda)$. Similarly the maximal nilideal of $\mathfrak{A}$ coincides with that of $\mathfrak{A}(\lambda)$ for every $\lambda \neq 1 / 2$. Finally if every nilideal of $\mathfrak{A}(\lambda)$ is solvable (strongly nilpotent) this is true for every nilideal of $\mathfrak{A}$.

The results just given imply that if $\mathfrak{A}(\lambda)$ has the property that when its 
maximal solvable ideal is zero it is a direct sum of simple algebras this will also be true of $\mathfrak{A}$. Moreover $\mathfrak{A}(\lambda)$ is simple if and only if $\mathfrak{A}$ is simple. In particular every simple associative algebra $\mathfrak{A}$ determines a class of flexible, Jordan-admissible and Lie-admissible simple algebras $\mathfrak{A}(\lambda)$. These are not commutative when $\mathfrak{A}$ is not commutative since $x \cdot y-y \cdot x=(2 \lambda-1)(x y-y x)$. They are also not associative, in general, even when $\mathfrak{A}$ is associative. This result follows for total matric algebras by use of the identity we shall derive in the next section.

2. Quasiassociative algebras. Let $\mathfrak{A}$ be an algebra over a field $\mathfrak{F}$ of characteristic not two. Then $\mathfrak{A}$ will be called a quasiassociative algebra if there exists a scalar extension $\Omega$ of $\mathfrak{F}$ and a quantity $\lambda$ in $\Omega$ such that $\mathfrak{A}_{\Re}=\mathfrak{B}(\lambda)$ where $\mathfrak{B}$ is an associative algebra over $\Re$. If $\lambda=0,1$ then $\mathfrak{A}_{\Re}$ is either isomorphic or antiisomorphic to $\mathfrak{B}$ and $\mathfrak{A}_{\Re}$ is associative, $\mathfrak{A}$ is associative. If $\lambda=1 / 2$ then $\mathfrak{A}_{\Re}$ is a Jordan algebra, $x y=y x$ and $x\left(y x^{2}\right)=(x y) x^{2}$ for every $x$ and $y$ of $\mathfrak{A}_{\Re}$, the same relations hold for every $x, y$ of $\mathfrak{A}, \mathfrak{A}$ is a Jordan algebra. Assume henceforth that $\lambda \neq 0,1,1 / 2$ whence $\mathfrak{B}=\mathfrak{A}_{\Re}(\mu)$ where $\mu=\lambda(2 \lambda-1)^{-1}$ and $\mathfrak{A}_{\Omega}(\mu)$ is associative. Evidently

$$
\mu \neq 0,1,1 / 2 \text {. }
$$

If $A$ is quasiassociative then $\mathfrak{B}(\lambda)=\mathfrak{A}_{\mathfrak{R}}$ is flexible, $x(y x)=(x y) x$ for every $x, y$ of $\mathfrak{A}, \mathfrak{A}$ is flexible. We now prove

THEOREM 5. Let $\mathfrak{A}$ be a quasiassociative algebra over a field $\mathfrak{F}$ of characteristic not two and let $\mathfrak{A}$ be neither associative nor a Jordan algebra. Then there exists $a$ quantity $\alpha_{0} \neq 0,-1 / 4$ in $\mathfrak{F}$ and a scalar extension $\mathfrak{\Omega}=\mathfrak{F}(\mu)$ of $\mathfrak{F}$ such that $\mu^{2}=\mu+\alpha_{0}, \mathfrak{A}_{\Omega}(\mu)$ is an associative algebra.

For the defining operation of $\mathfrak{A}_{\Omega}(\mu)$ is $x \cdot y=\mu x y+(1-\mu) y x$ where $x y$ is the product in $\mathfrak{A}$ and in $\mathfrak{A}_{\mathfrak{\Omega}}$. We compute

$$
x \cdot(y \cdot z)=\mu x[\mu(y z)+(1-\mu) z y]+(1-\mu)[\mu y z+(1-\mu) z y] x
$$

and

$$
(x \cdot y) \cdot z=\mu[\mu x y+(1-\mu) y x] z+(1-\mu) z[\mu x y+(1-\mu) y x]
$$

to see that $\mathfrak{A}_{\mathfrak{R}}(\mu)$ is associative if and only if $\mu^{2}[x(y z)-(x y) z]+(1-\mu)^{2}$ $[(z y) x-z(y x)]+\mu(1-\mu)[x(z y)+(y z) x-(y x) z-z(x y)]=0$ for every $x, y, z$ of $\mathfrak{A}_{\Omega}$. However $\mathfrak{A}_{\Omega}$ is flexible and so

$$
x(y z)-(x y) z=(z y) x-z(y x) .
$$

It follows that $\mathfrak{A}_{\Omega}(\mu)$ is associative if and only if $\mathfrak{A}_{\Re}$ is flexible and

$$
\left[\mu^{2}+(1-\mu)^{2}\right][x(y z)-(x y) z]=\left(\mu^{2}-\mu\right)[x(z y)+(y z) x-(y x) z-z(x y)]
$$

for every $x, y, z$ of $\mathfrak{A}_{\Omega}$. Since this relation is linear in $x, y, z$ it holds for every $x, y, z$ of $\mathfrak{A}_{\Re}$ if and only if it holds for every $x, y, z$ of $\mathfrak{A}$. Define $\alpha_{0}=\mu^{2}-\mu$ and see that $\mathfrak{A}_{\mathfrak{R}}(\mu)$ is associative if and only if $\mathfrak{A}$ is flexible and 


$$
\left(2 \alpha_{0}+1\right)[x(y z)-(x y) z]=\alpha_{0}[x(z y)+(y z) x-(y x) z-z(x y)]
$$

for every $x, y, z$ of $\mathfrak{A}$. If $\alpha_{0}$ were not in $\mathfrak{F}$ then a sum $a+b \alpha_{0}=0$ for $a$ and $b$ in $\mathfrak{A}$ only if $a=b=0$, (5) would imply that $x(y z)=(x y) z$, that is, $\mathfrak{A}$ would be associative. It follows that $\alpha_{0}=\mu^{2}-\mu$ is in $\mathfrak{F}$ and the hypothesis (1) implies that $\alpha_{0} \neq 0,-1 / 4$. This proves our theorem.

As an immediate corollary of our proof we have

THEOREM 6. Let $\mathfrak{A}$ be an algebra over a field $\mathfrak{F}$ of characteristic not two and let $\mathfrak{A}$ be neither associative nor a Jordan algebra. Then $\mathfrak{A}$ is quasiassociative if and only if $\mathfrak{A}$ is flexible and there exists an element $\alpha_{0} \neq 0,-1 / 4$ in $\mathfrak{F}$ such that (5) holds for every $x, y, z$ of $\mathfrak{A}$.

Let us observe that if $\mathfrak{A}$ is associative and $\mu \neq 0,1$ we may use (2) and (3) to see that $\mathfrak{A}(\mu)$ is associative if and only if $x(z y)+(y z) x=(y x) z+z(x y)$, that is, $(x z-z x) y=y(x z-z x)$. Then $\mathfrak{A}(\mu)$ is associative if and only if $x z-z x$ is in the center of $\mathfrak{A}$ for every $x$ and $z$ of $\mathfrak{A}$. But if $\mathfrak{A}$ is a total matric algebra of degree $n>1$ over $\mathfrak{F}$ we may use the usual basis $e_{i j}$ of $\mathfrak{A}$ with $x=e_{12}, z=e_{21}$ to see that $x z-z x=e_{11}-e_{22}$ which is not in the center of $\mathfrak{A}$ since $e_{12}\left(e_{11}-e_{22}\right)$ $=-e_{12},\left(e_{11}-e_{12}\right) e_{12}=e_{12}$.

3. Defining identities. A quasiassociative algebra $\mathfrak{A}$ which is neither associative nor a Jordan algebra is defined by the existence of an element $\alpha_{0} \neq 0,-1 / 4$ such that $x(y x)=(x y) x$ and (5) holds. Conversely if (5) and the relation $x(y x)=(x y) x$ hold the corresponding algebra is quassiassociative. It should be noted that associative and Jordan algebras do not, in general, satisfy (5).

We may write (5) in the form

$$
\left(2 \alpha_{0}+1\right)\left(R_{y z}-R_{y} R_{z}\right)=\alpha_{0}\left(R_{z y}+L_{y z}-L_{y} R_{z}-R_{y} L_{z}\right) .
$$

By the flexible law $L_{y z}=R_{z y}-R_{z} R_{y}+L_{z} L_{y}$ and so (6) becomes

(7) $2 \alpha_{0}\left(R_{y z}-R_{z y}\right)+R_{y z}=\left(2 \alpha_{0}+1\right) R_{y} R_{z}+\alpha_{0}\left(L_{z} L_{y}-R_{z} R_{y}-L_{y} R_{z}-R_{y} L_{z}\right)$.

Interchange $y$ and $z$ to obtain

(8) $2 \alpha_{0}\left(R_{z y}-R_{y z}\right)+R_{z y}=\left(2 \alpha_{0}+1\right) R_{z} R_{y}+\alpha_{0}\left(L_{y} L_{z}-R_{y} R_{z}-L_{z} R_{y}-R_{z} L_{y}\right)$.

Add and use $R_{y} L_{z}+R_{z} L_{y}=L_{y} R_{z}+L_{z} R_{y}$ (by (4.2)) to obtain

(9) $R_{y z+z y}=\left(\alpha_{0}+1\right)\left(R_{y} R_{z}+R_{z} R_{y}\right)+\dot{\alpha_{0}}\left(L_{y} L_{z}+L_{z} L_{y}\right)-2 \alpha_{0}\left(R_{y} L_{z}+R_{z} L_{y}\right)$.

We may also subtract (8) from (7) to obtain

$$
\begin{aligned}
\left(4 \alpha_{0}+1\right) R_{y z-z y}= & \left(3 \alpha_{0}+1\right)\left(R_{y} R_{z}-R_{z} R_{y}\right)+\alpha_{0}\left(L_{z} L_{y}-L_{y} L_{z}\right) \\
& +\alpha_{0}\left(L_{z} R_{y}+R_{z} L_{y}-L_{y} R_{z}-R_{y} L_{z}\right)
\end{aligned}
$$

and from (5) with $x$ and $y$ interchanged we have

$$
\left(2 \alpha_{0}+1\right)\left(R_{z} L_{y}-L_{y} R_{z}\right)=\alpha_{0}\left(L_{z} L_{y}+R_{z} R_{y}-R_{y} R_{z}-L_{y} L_{z}\right) .
$$


Finally, by (4.2) we may write

$$
R_{y} L_{z}-L_{z} R_{y}=L_{y} R_{z}-R_{z} L_{y}
$$

If $\alpha_{0}=-1 / 2$, then $L_{z} L_{y}-L_{y} L_{z}=R_{y} R_{z}-R_{z} R_{y}$, and so (7) reduces to

$$
R_{z y}=-\left(L_{z} L_{y}-R_{z} R_{y}-L_{y} R_{z}-R_{y} L_{z}\right) / 2 .
$$

Otherwise we have $\alpha_{0}\left(L_{z} R_{y}+R_{z} L_{y}-L_{y} R_{z}-R_{y} L_{z}\right)=2 \alpha_{0}\left(R_{z} L_{y}-L_{y} R_{z}\right)$. Thus $\left(4 \alpha_{0}+1\right) R_{y z-z y}=\left(3 \alpha_{0}+1\right)\left(R_{y} R_{z}-R_{z} R_{y}\right)+\alpha_{0}\left(L_{z} L_{y}-L_{y} L_{z}\right)+2 \alpha_{0}\left(R_{z} L_{y}-L_{y} R_{z}\right)$ $=\left(4 \alpha_{0}+1\right)\left(R_{y} R_{z}-R_{z} R_{y}\right)+2 \alpha_{0}\left(R_{z} L_{y}-L_{y} R_{z}\right), \alpha_{0}\left(L_{z} L_{y}-L_{y} L_{z}+R_{z} R_{y}-R_{y} R_{z}\right)$ and so

$$
\left(4 \alpha_{0}+1\right) R_{y z-z y}=\left(4 \alpha_{0}+1\right)\left(R_{y} R_{z}-R_{z} R_{y}\right)+\left(4 \alpha_{0}+1\right)\left(R_{z} L_{y}-L_{y} R_{z}\right)
$$

which implies

$$
R_{y z-z y}=\left(R_{y} R_{z}-R_{z} R_{y}\right)+\left(L_{z} R_{y}-R_{y} L_{z}\right) .
$$

Thus we have

$$
\begin{aligned}
2 R_{y z}= & \left(\alpha_{0}+2\right)\left(R_{y} R_{z}-R_{z} R_{y}\right)+\alpha_{0}\left(L_{y} L_{z}+L_{z} L_{y}\right) \\
& -2 \alpha_{0}\left(R_{y} L_{z}+R_{z} L_{y}\right)+L_{z} R_{y}-R_{y} L_{z} .
\end{aligned}
$$

This proves the following theorem.

Theorem 7. Let $\mathfrak{A}$ be a quasiassociative algebra. Then $\mathfrak{A}$ is shrinkable of level one.

4. J-semi-simple algebras. An algebra $\mathfrak{A}$ will be called J-semi-simple if $\mathfrak{A}^{(+)}$is a semi-simple Jordan algebra and will be called J-simple if $\mathfrak{A}^{(+)}$is a simple Jordan algebra.

THEOREM 8. Every $J$-semi-simple algebra is a direct sum $\mathfrak{A}=\mathfrak{A}_{1} \oplus \cdots \oplus \mathfrak{A}_{r}$ where the components $\mathfrak{A}_{i}$ are $J$-simple. Every $J$-simple algebra $\mathfrak{A}$ is simple and the center of $\mathfrak{A}$ is the center of $\mathfrak{A}^{(+)}$.

For, if $\mathfrak{B}$ is an ideal of the semi-simple Jordan algebra $\mathfrak{A}^{(+)}$then $\mathfrak{B}$ has a unity element $e$ and $\mathfrak{A}^{(+)}=\mathfrak{B} \oplus \mathfrak{S}, \mathfrak{B}=\mathfrak{H}_{e}^{(+)}(1), \quad \mathfrak{S}=\mathfrak{A}_{e}^{(+)}(0)$. It follows that $\mathfrak{A}=\mathfrak{A}_{e}(1) \oplus \mathfrak{A}_{e}(0)$ and that $\mathfrak{B}=\mathfrak{A}_{e}(1)^{(+)}, \mathfrak{E}=\mathfrak{H}_{e}(0)^{(+)}$. If we decompose $\mathfrak{A}^{(+)}$ as a direct sum $\mathfrak{Y}^{(+)}=\mathfrak{B}_{1} \oplus \cdots \oplus \mathfrak{B}_{t}$ of simple Jordan algebras $\mathfrak{B}_{i}$ then each $\mathfrak{B}_{i}=\mathfrak{A}_{e_{i}}^{(+)}(1)=\mathfrak{A}_{i}^{(+)}$where $\mathfrak{A}=\mathfrak{A}_{1} \oplus \cdots \oplus \mathfrak{A}_{t}$, the algebras $\mathfrak{A}_{i}$ are J-simple. Assume now that $\mathfrak{A}$ is $\mathrm{J}$-simple. Then every ideal $\mathfrak{M}$ of $\mathfrak{A}$ is also an ideal of $\mathfrak{A}^{(+)}$and if $\mathfrak{M} \neq 0$ we have $\mathfrak{M}=\mathfrak{A}$. Hence $\mathfrak{A}$ is simple. If $\mathfrak{Z}$ is the center of $\mathfrak{A}$ we may express $\mathfrak{A}$ as an algebra over $\mathfrak{Z}$. But then $\mathfrak{U}^{(+)}$will be an algebra over 8 and so 3 is contained in the center of $\mathfrak{A}^{(+)}$.However it is true for arbitrary simple algebras that $\mathfrak{A}$ is central simple over $\mathbb{B}$ if and only if every scalar extension over $\mathbb{B}$ of $\mathfrak{A}$ is a simple algebra. Thus if $B$ is not the center of $\mathfrak{A}^{(+)}$ there will be a scalar extension $\Re$ of $\mathbb{Z}$ such that $\mathfrak{A}_{\Re}^{(+)}=\mathfrak{B} \oplus \mathfrak{C}$. By the proof above $\mathfrak{A}_{\Re}$ is a direct sum of the corresponding components contrary to the 
hypothesis that $\mathbb{Z}$ is the center of $\mathfrak{A}$ and so $\mathfrak{A}_{\Re}$ is simple for every $\Re$ over $\mathfrak{Z}$.

5. The structure of quasiassociative algebras. The general theorems on the structure of quasiassociative algebras may be stated as follows:

Theorem 9. Let $\mathfrak{A}$ be a quasiassociative algebra over an infinite field $\mathfrak{F}$ of characteristic not two. Then if $\mathfrak{A}$ is solvable it is strongly nilpotent and if $\mathfrak{A}$ is a nilalgebra it is solvable.

THEOREM 10. Every quasiassociative algebra $\mathfrak{A}$ over a field $\mathfrak{F}$ contains a maximal solvable ideal $\mathfrak{N}$ called the radical of $\mathfrak{A}$, such that every nilideal of $\mathfrak{A}$ is contained in $\mathfrak{N}$. If $\mathfrak{F}$ is nonmodular and $\mathfrak{W}$ is any scalar extension of $\mathfrak{F}$ the radical of $\mathfrak{A}_{\mathfrak{B}}$ is $\mathfrak{N}_{\mathfrak{F}}$. Also $\mathfrak{A}-\mathfrak{N}$ is semi-simple, that is, $\mathfrak{A}-\mathfrak{N}$ has no nonzero nilideal.

Theorem 11. A semi-simple quasiassociative algebra is $J$-simi-simple and so is a direct sum $\mathfrak{U}=\mathfrak{A}_{1} \oplus \cdots \oplus \mathfrak{A}_{r}$ of $J$-simple algebras $\mathfrak{A}_{i}$. Each $\mathfrak{A}_{i}$ is quasiassociative and so is flexible. A quasiassociative simple algebra with center 8 is quasiassociative over 3 .

The results just stated clearly hold for Jordan algebras and for associative algebras. Theorems 9 and 10 have been seen to hold for those algebras $\mathfrak{A}$ for which there exists an element $\lambda$ in $\mathfrak{F}$ such that $\lambda \neq 0,1,1 / 2$ and $\mathfrak{A}(\lambda)$ is associative. Evidently Theorem 11 also holds for such algebras. There remains the case $\mathfrak{U}_{\Re}=\mathfrak{B}(\mu)$ for a quadratic field $\Re=\mathfrak{F}(\mu)$ over $\mathfrak{F}$ and an associative algebra $\mathfrak{B}$ over $\Re$. Note that the properties above already hold for $\mathfrak{A}_{\Omega}$.

Let $\mu^{2}=\mu+\alpha_{0}$ for $\alpha_{0}$ in $\mathfrak{F}$ and write $\theta=2 \mu-1$ so that $\theta^{2}=4\left(\mu^{2}-\mu\right)+1$ $=4 \alpha_{0}+1$ is in $\mathfrak{F}$ and must not be the square of any element of $\mathfrak{F}$. Then every vector subspace $\mathfrak{M}$ over $\mathfrak{R}$ of $\mathfrak{A}_{\mathfrak{R}}$ has elements of the form $x=x_{0}+x_{1} \theta$ where $x_{0}$ and $x_{1}$ are in $\mathfrak{A}$. If $y=y_{0}+y_{1} \theta$ is in $\mathfrak{M}$ and $\xi$ and $\eta$ are in $\mathfrak{F}$ the element $\xi x+\eta y=\left(\xi x_{0}+\eta y_{0}\right)+\left(\xi x_{1}+\eta y_{1}\right) \theta$ is in $\mathfrak{M}$. Hence if $\mathfrak{M}_{0}$ is the set of all $x_{0}$ then $\mathfrak{M}_{0}$ is a vector subspace over $\mathfrak{F}$ of $\mathfrak{A}$. But $\theta^{-1} x=x_{1}+\left(4 \alpha_{0}+1\right)^{-1} x_{0} \theta$ is in $\mathfrak{M}$ and so $x_{1}$ is in $\mathfrak{M}_{0}, \mathfrak{M} \leqq\left(\mathfrak{M}_{0}\right)_{\mathfrak{R}}$.

Suppose now that $\mathfrak{M}$ is an ideal of $\mathfrak{A}_{\Re}$. Then if $y$ is in $\mathfrak{A}$ and $x=x_{0}+x_{1} \theta$ is in $\mathfrak{M}$ the products $x y=x_{0} y+\left(x_{1} y\right) \theta$ and $y x=y x_{0}+\left(y x_{1}\right) \theta$ are in $\mathfrak{M}, x_{0} y$ and $y x_{0}$ are in $\mathfrak{M}_{0}, \mathfrak{M}_{0}$ is an ideal of $\mathfrak{A}$. Thus every ideal $\mathfrak{M}$ of $\mathfrak{A}_{\mathfrak{R}}$ determines an ideal $\mathfrak{M}_{0}$ of $\mathfrak{A}$ such that $\mathfrak{M} \leqq\left(\mathfrak{M}_{0}\right)_{\mathfrak{R}}$. Conversely every ideal $\mathfrak{M}_{0}$ of $\mathfrak{A}$ determines an ideal $\left(\mathfrak{M}_{0}\right)_{\Re}$ of $\mathfrak{A}_{\Re}$.

Assume now that $\mathfrak{A}$ is solvable. Then $\mathfrak{A}_{\Omega}$ is solvable. Indeed if we define $\mathfrak{A}^{(k+1)}=\mathfrak{A}^{(k)} \mathfrak{A}^{(k)}$ then $\left[\mathfrak{A}_{\mathfrak{B}}\right]^{(k+1)}=\left[\mathfrak{A}_{\mathfrak{B}}\right]^{(k)}\left[\mathfrak{P}_{\mathfrak{W}}\right]^{(k)}$ for every scalar extension $\mathfrak{W}$ of $\mathfrak{F}$ and $\mathfrak{A}^{(k)}=0$ implies that $\left[\mathfrak{A}_{\mathfrak{B}}\right]^{(k)}=0$. But then $\mathfrak{A}_{\mathfrak{R}}$ is strongly nilpotent, that is, every product of $t$ elements of $\mathfrak{A}_{\Re}$ is zero for some positive integer $t$. Clearly then $\mathfrak{A}$ is strongly nilpotent. If $\mathfrak{A}$ is a nilalgebra and $u_{1}, \cdots, u_{n}$ form a basis of $\mathfrak{A}$ then $\left(\xi_{1} u_{1}+\cdots+\xi_{n} u_{n}\right)^{n}=0$ for $\xi_{1}, \cdots, \xi_{n}$ indeterminates over $\mathfrak{F}$. Otherwise we could find values in $\mathfrak{F}$ of $\xi_{1}, \cdots, \xi_{n}$ such that the corresponding element of $\mathfrak{A}$ is not nilpotent. But then we may replace the $\xi_{i}$ by any elements of $\mathfrak{R}, \mathfrak{A}_{\Re}$ must be a nilalgebra, $\mathfrak{A}_{\Re}$ is strongly nilpotent, so is $\mathfrak{A}$. 
The first statement in Theorem 10 holds by virtue of Theorem 9. To prove the second part we first prove that if $\mathfrak{F}$ is nonmodular with $\mathfrak{N}$ and $\mathfrak{M}$ the respective radicals of $\mathfrak{U}$ and $\mathfrak{A}_{\Omega}$ then $\mathfrak{N}_{\Re}=\mathfrak{M}$. Evidently $\mathfrak{R}_{\Re} \leqq \mathfrak{M}$. Conversely let $x$ be in $\mathfrak{M}, T$ be any transformation of the algebra of polynomials in the multiplications of the elements of $\mathfrak{A}$ and $y=x T$. Then $y$ is in $\mathfrak{M}$ and so $a R_{y}^{k+1}=(a y) R_{y}^{k}$ is a product of $k+1$ elements of $\mathfrak{M}$ and is zero for every $a$ of $\mathfrak{A}_{\Re}$ and a suitable $k, R_{y}^{k+1}=0$. Similarly $a L_{y}^{k+1}=(y a) L_{y}^{k}=0, R_{y}$ and $L_{y}$ are nilpotent and have zero trace. Write the general element $x$ of $\mathfrak{M}$ as $x=x_{0}$ $+x_{1} \theta$ and see that $y=x R_{x_{0}}^{k}=x_{0}^{k+1}+\left(x_{1} R_{x_{0}}^{k}\right) \theta=u+v \theta, \tau\left(R_{y}\right)=\tau\left(R_{u}\right)+\theta \tau\left(R_{v}\right)=0$ so that $\tau\left(R_{u}\right)=0$. Similarly $\tau\left(L_{u}\right)=0$ and so the linear transformation $S_{u}=\left(R_{u}+L_{u}\right) / 2$ has zero trace for $u=x_{0}^{k+1}$ and every positive integer $k$. But $x_{0}$ is in the Jordan algebra $\mathfrak{Y}_{\Re}^{(+)}, S_{u}$ is the right multiplication for $x_{0}^{k+1}$ and has zero trace. If $\mathfrak{F}\left[x_{0}^{2}\right]$ were to contain an idempotent $e$, we would have $\tau\left(S_{e}\right) \neq 0$ whereas $e$ is a polynomial in $x_{0}^{2}$ and $S_{x}$ is a linear function of $x$. Thus there is no such idempotent, $x_{0}^{2}$ is nilpotent, $x_{0}$ is nilpotent, the ideal $\mathfrak{M}_{0}$ defined above is a nilideal of $\mathfrak{A}, \mathfrak{M}_{0} \leqq \mathfrak{N}, \mathfrak{M} \leqq\left(\mathfrak{M}_{0}\right)_{\mathfrak{R}} \leqq \mathfrak{N}_{\Re}, \mathfrak{M}=\mathfrak{N}_{\Omega}$.

To prove Theorem 11 we note that if $\mathfrak{A}$ is a semi-simple quasiassociative algebra, then $\mathfrak{A}_{\mathfrak{R}}$ is semi-simple and so the related associative algebra $\mathfrak{B}$ is semi-simple. But then $\mathfrak{B}^{(+)}$is semi-simple, $\mathfrak{B}^{(+)}=\mathfrak{U}_{\mathfrak{R}}^{(+)}=\left(\mathfrak{Y}^{(+)}\right)_{\mathfrak{R}}$ is a semisimple Jordan algebra. It follows that $x\left(y x^{2}\right)=(x y) x^{2}$ for every $x$ and $y$ of the commutative algebra $\mathfrak{A}^{(+)}$and so $\mathfrak{X}^{(+)}$is a semi-simple Jordan algebra. Write $\mathfrak{U}=\mathfrak{A}_{1} \oplus \cdots \oplus \mathfrak{A}_{r}$ and see that $\mathfrak{A}_{\mathfrak{R}}=\left(\mathfrak{A}_{1}\right)_{\mathfrak{R}} \oplus \cdots \oplus\left(\mathfrak{A}_{r}\right)_{\mathfrak{R}}$. However if $x y=y x=0$ then $x \cdot y=\lambda x y+(1-\lambda) y x=y \cdot x=0$ and so $\mathfrak{A}_{\Re}(\lambda)=\left(\mathfrak{A}_{1}\right)_{\mathfrak{R}}(\lambda) \oplus \cdots$ $\oplus\left(\mathfrak{A}_{r}\right)_{\mathfrak{R}}(\lambda)$ where $\left(\mathfrak{A}_{\mathfrak{i}}\right)_{\mathfrak{R}}(\lambda)$ must be associative. Assume, finally, that $\mathfrak{A}$ is a simple quasiassociative algebra with center 3 and that $\mathfrak{A}_{\Omega}(\lambda)$ is associative where $\Re=\mathfrak{F}(\lambda), \lambda^{2}-\lambda=\alpha_{0}$ in $\mathfrak{F}$. If $\lambda$ is in $\mathfrak{F}$ then $\mathfrak{A}$ is clearly quasiassociative over $\mathbb{Z}$. Hence let $\Omega$ have degree two over $\mathfrak{F}$. Then either the composite of $\mathbb{Z}$ and $\Omega$ is a field $B(\lambda)$ of degree two over $B$ and $\mathfrak{A}$ is quasiassociative over $\mathbb{Z}$ or $\mathbb{Z}$ has a quadratic subfield $\mathfrak{W}$ isomorphic over $\mathfrak{F}$ to $\Omega$. Then $\mathfrak{W}_{\Omega}=e_{1} \Omega+e_{2} \Omega$ where $e_{1}$ and $e_{2}$ are pairwise orthogonal idempotents, $\mathfrak{A}_{\Re}=\mathfrak{B}_{1} \oplus \mathfrak{B}_{2}$ where $\mathfrak{B}_{1}=\mathfrak{A}_{\Re} e_{1}$ and $\mathfrak{B}_{2}=\mathfrak{U}_{\Re} e_{2}$. Let $u_{1}, \cdots, u_{n}$ be a basis of $\mathfrak{A}$ over $\mathfrak{W}$ and let $u_{i} u_{j}=\sum \gamma_{i j k} u_{k}$ where the $\gamma_{i j k}$ are in $\mathfrak{B}$. Then $\left(u_{i} e_{1}\right)\left(u_{j} e_{1}\right)=\left(u_{i} u_{j}\right) e_{1}$ $=\sum\left(\gamma_{i j k} e_{1}\right) u_{k} e_{1}$ where the elements $u_{i} e_{1}$ are a basis of $\mathfrak{B}_{1}$. Moreover $\mathfrak{B}=\mathfrak{F}(x)$ where $x^{2}=\beta_{0}$ in $\mathfrak{F}, \quad \Re=\mathfrak{F}(\theta)$ where $\theta^{2}=\beta_{0}, x=\theta e_{1}-\theta e_{2}, x e_{1}=\theta e_{1}, \gamma_{i j k}(x) e_{1}$ $=\gamma_{i j k}(\theta) e_{1}$. It follows that $\mathfrak{B}_{1}$ is an algebra of order $2 n$ over $\mathfrak{F}$ isomorphic to $\mathfrak{A}$ over $\mathfrak{F}$ under a mapping which maps $x$ on $\theta e_{1}$. Evidently $\mathfrak{A}_{\Re}(\lambda)=\mathfrak{B}_{1}(\lambda)$ $\oplus \mathfrak{B}_{2}(\lambda)$ and so $\mathfrak{B}_{1}(\lambda)$ is associative, $\mathfrak{A}((x+1) / 2)$ is associative. $\mathfrak{A}$ is quasiassociative over 3 .

We have now reduced the study of quasiassociative algebras to the case of central simple quasiassociative algebras $\mathfrak{A}$ such that $\mathfrak{A}^{(+)}$is a central simple Jordan algebra over $\mathfrak{F}$. Then $\mathfrak{A}_{\Re}=\mathfrak{B}(\lambda)$ where $\mathfrak{R}=\mathfrak{F}(\lambda)$ has degree one or two over $\mathfrak{F}$ and $\mathfrak{B}$ is a central simple associative algebra over $\Re$. It is not true, in general, that there always exists an element $\lambda$ in $\mathfrak{F}$ such that $\mathfrak{A}=\mathfrak{B}(\lambda)$. For we shall later show exactly when this is true for algebras of degree two and 
order four. However it might be true that there always exists an associative central simple algebra $\mathfrak{B}$ over $\mathfrak{F}$ such that $\mathfrak{A}_{\Re}=\mathfrak{B}_{\Re}(\lambda)$ and we leave this as an unsolved problem.

6. Algebras of degree two. Let $\mathfrak{A}$ be a flexible J-simple algebra of degree two over a field $\mathfrak{F}$ of characteristic not two. Then $\mathfrak{U}$ has a unity element $e$ and a basis $u_{1}=e, u_{2}, \cdots, u_{n}$ where $n \geqq 3, u_{i}^{2}=\alpha_{i} e$ for $\alpha_{i} \neq 0$ in $\mathfrak{F}, u_{i} u_{j}+u_{j} u_{i}$ $=0$ for $i \neq j$ and $i, j=2, \cdots, n$. It follows that the general element $x=\xi_{1} u_{1}+\cdots+\xi_{n} u_{n}$ of $\mathfrak{A}$ satisfies the equation $\left(x-\xi_{1} e\right)^{2}=f(x) e, f(x)=\alpha_{2} \xi_{2}^{2}$ $+\cdots+\alpha_{n} \xi_{n}^{2}$.

Assume the multiplication table $u_{i} u_{j}=\sum_{k=1}^{n} \gamma_{i j k} u_{k}$ for $\gamma_{i j k}$ in $\mathfrak{F}$. It is evidently necessary only to study the elements $\gamma_{i j k}$ for $i \neq j$ and $i, j=2, \cdots, n$. The condition $u_{i} u_{j}=-u_{j} u_{i}$ is expressed by

$$
\gamma_{i j k}=-\gamma_{j i k} \quad(i \neq j ; i, j=2, \cdots, n) .
$$

The assumption that $\mathfrak{A}$ is flexible is now equivalent to the relation $x(y z)$ $+z(y x)=(x y) z+(z y) x$ for $x, y, z$ taken to be basal elements $u_{i}$ for $i>1$. In case two of the variables are equal we have as one case $u_{i}\left(u_{i} u_{j}\right)+u_{j}\left(u_{i} u_{i}\right)=\left(u_{i} u_{i}\right) u_{j}$ $+\left(u_{j} u_{i}\right) u_{i}$, that is, $u_{i}\left(u_{i} u_{j}\right)+\left(u_{i} u_{j}\right) u_{i}=0$. Then $2 \gamma_{i j 1} u_{i}+2 \gamma_{i j i} \alpha_{i} e=0$. Similarly $u_{j}\left(u_{j} u_{i}\right)+\left(u_{j} u_{i}\right) u_{j}=0$, that is, $2 \gamma_{i j 1} u_{j}+2 \gamma_{i j j} \alpha_{j} e=0$. This yields

$$
\gamma_{i j 1}=\gamma_{i j i}=\gamma_{i j j}=0
$$$$
(i \neq j ; i, j=2, \cdots, n) \text {, }
$$

a result stating that every product of two distinct basal elements $u_{i}, u_{j}$ is a linear combination of the basal elements $u_{k}$ distinct from $e, u_{i}$ and $u_{j}$.

The flexibility relation used above may now be employed with $x=z=u_{j}$ and $y=u_{i}$ to yield $u_{j}\left(u_{i} u_{i}\right)=\left(u_{j} u_{i}\right) u_{j}$. This is equivalent to $u_{j}\left(u_{i} u_{i}\right)+\left(u_{i} u_{j}\right) u_{j}$ $=0$, a relation already satisfied by $(18)$. Thus there remains only the case where $x=u_{i}, y=u_{j}, z=u_{k}$ with $i, j, k$ all distinct. The relation becomes $u_{i}\left(u_{j} u_{k}\right)+u_{k}\left(u_{j} u_{i}\right)=\left(u_{i} u_{j}\right) u_{k}+\left(u_{k} u_{j}\right) u_{i}$ which is equivalent to $u_{i}\left(u_{j} u_{k}\right)$ $+\left(u_{j} u_{k}\right) u_{i}=\left(u_{i} u_{j}\right) u_{k}+u_{k}\left(u_{i} u_{j}\right)$ by using skew-commutivity. Hence we have

$$
\alpha_{i} \gamma_{j k i}=\alpha_{k} \gamma_{i j k} \quad(i \neq j ; i, j, k=2, \cdots, n)
$$

where (19) is satisfied by (18) in case $k=i$ or $k=j$.

We have now determined a set of conditions which are necessary and sufficient conditions that an algebra $\mathfrak{A}$ of degree two shall be both flexible and $\mathrm{J}$-simple. A complete normalization of the multiplication table of the general algebra of order $n$ of our type is manifestly impossible and is not even particularly significant for algebras of order $n>4$. We shall therefore content ourselves with a discussion of algebras of order three and four.

When $n=3$ the product $u_{2} u_{3}=0$ by (18) and so $u_{3} u_{2}=0, \mathfrak{A}$ is commutative and is a Jordan algebra.

Assume that $n=4$ and use the notations $u=u_{2}, v=u_{3}, w=u_{4}, u^{2}=\alpha e$, $v^{2}=\beta e, w^{2}=\gamma e$ where $\alpha=\alpha_{2} \neq 0, \beta=\alpha_{3} \neq 0$ and $\gamma=\alpha_{4} \neq 0$ are in $\mathfrak{F}$. Then $u v=\rho w$ where $\rho=\gamma_{234}$ by (18). Apply (19) to see that $\alpha_{4} \gamma_{234}=\alpha_{2} \gamma_{342}$ and so 
$\gamma_{342}=\alpha^{-1} \gamma \rho$, vw $=\alpha^{-1} \gamma \rho u$. Also $\alpha_{3} \gamma_{243}=-\alpha_{3} \gamma_{423}=-\alpha_{4} \gamma_{234}, \gamma_{243}=-\beta^{-1} \gamma \rho$, uw $=-\beta^{-1} \gamma \rho v$. When $\rho=0$ the algebra so defined is the general simple Jordan algebra of degree two and order four over $\mathfrak{F}$. When $\rho \neq 0$ we may select a new basis with $w=u v$ and so take $\rho=1$. Then we have the multiplication table

$$
u^{2}=\alpha e, v^{2}=\beta e, w^{2}=\gamma e, u v=w, u w=-\beta^{-1} \gamma v, v w=\alpha^{-1} \gamma u .
$$

When $\mathfrak{A}$ is associative $u w=u(u v)=u^{2} v=\alpha v$, that is, $\gamma=-\alpha \beta$. It follows that the algebra defined by (20) is associative if and only if $\gamma=-\alpha \beta$.

It remains to study the quasiassociativity of $\mathfrak{A}$. Define an algebra $\mathfrak{B}$ with a basis $e_{0}, u_{0}, v_{0}, w_{0}$ and the associative multiplication table given by the assumption that $e_{0}$ is the unity quantity of $\mathfrak{B}$ and the relations $u_{0}^{2}=\alpha e_{0}$, $v_{0}^{2}=\beta e_{0}, w_{0}^{2}=-\alpha \beta e_{0}, w_{0}=u_{0} \cdot v_{0}$. Form $\mathfrak{B}(\lambda)$ by defining a new product $x y=\lambda x \cdot y+(1-\lambda) y \cdot x$ and see that $u_{0} v_{0}=\lambda w_{0}+(1-\lambda)\left(-w_{0}\right)=(2 \lambda-1) w_{0}$, $\left(u_{0} v_{0}\right)^{2}=\gamma e_{0}$ if and only if $\gamma=-(2 \lambda-1)^{2} \alpha \beta$. Since $\mathfrak{B}$ is associative the algebra $\mathfrak{B}(\lambda)$ is flexible and the relation $\left(u_{0} v_{0}\right)^{2}=\gamma e_{0}$ is sufficient to imply that $\mathscr{B}(\lambda)$ is isomorphic to the given algebra $\mathfrak{A}$ of (20). It follows that $\mathfrak{A}$ is isomorphic to some $\mathfrak{B}(\lambda)$ if and only if $-\alpha \beta \gamma$ is the square of an element of $\mathfrak{F}$. When $-\alpha \beta \gamma$ is not such a square we have $\mathfrak{A}_{\Re}$ isomorphic to $\mathfrak{B}_{\Re}(\lambda)$ where $\Re=\mathfrak{F}(\lambda)$ and $(2 \lambda-1)^{2}=-(\alpha \beta)^{-1} \gamma$, so that $\Omega$ is a quadratic field over $\mathfrak{F}$. We have proved that all flexible J-simple algebras of degree two and order four over their centers are quasiassociative. Since the order of a simple associative algebra of degree two over its center is necessarily four the flexible J-simple algebras of degree two and order $n>4$ are necessarily not quasiassociative.

One of the major problems in the study of nonassociative algebras is the question as to the existence of real nonassociative division algebras of order $2^{t}$ with $t>3$. A special case of this problem is the same question for flexible algebras of degree two. These algebras are necessarily J-simple and their study may be expedited by use of the following result:

Theorem 12. Let $\mathfrak{A}$ be a flexible J-simple algebra of degree two over $\mathfrak{F}$ of characteristic not two. Then $\mathfrak{A}$ is a division algebra if and only if $\mathfrak{A}(\lambda)$ is a division algebra for every $\lambda \neq 1 / 2$ in $\mathfrak{F}$.

To demonstrate this result we first note that when $\mathfrak{A}$ is a division algebra its associative subalgebras $\mathfrak{F}[x]$ must all be fields. This occurs if and only if the norm form $N(x)=\xi_{1}^{2}-\left(\alpha_{2} \xi_{2}^{2}+\cdots+\alpha_{n} \xi_{n}^{2}\right)$ is not a null form. We next prove the

Lemma. Let $N(x)$ be a non-null form. Then $\mathfrak{A}$ is a division algebra if and only if there exist no elements $x$ and $y$ in $\mathfrak{A}$ such that $x^{2}=N(x) e \neq 0, y^{2}=N(y) e \neq 0$, $x y=-y x=0$.

For when $\mathfrak{A}$ is not a division algebra there exist nonzero quantities $a$ and $b$ in $\mathfrak{A}$ such that $a b=0$. Since $a$ and $b$ are necessarily not in the same subfield of 
$\mathfrak{A}$ we may always write $a=\xi e+x, b=\eta e+\lambda x+y$ where $\xi, \eta, \lambda$ are in $\mathfrak{F}$ and $x^{2}=N(x) e \neq 0, y^{2}=N(y) e \neq 0, y x=-x y$. Then we may select a basis of $\mathfrak{A}$ with $u_{2}=x, u_{3}=y$ and so see that $x y$ is linearly independent of $e, x, y$. But $a b=(\xi \eta+N(x)) e+(\lambda \xi+\eta) x+\xi y+x y=0$ so that $x y=0$ as desired.

To complete our proof we observe that if $\mathfrak{A}(\lambda)$ is not a division algebra then there exist elements $x$ and $y$ in $\mathfrak{A}$ such that $x \cdot y=-y \cdot x=0$. But $x y=\mu x \cdot y$ $+(1-\mu) y \cdot x$ where $\mu=\lambda(2 \lambda-1)^{-1}, x y=(2 \mu-1) x \cdot y=0, \mathfrak{A}$ cannot be a division algebra.

7. Algebras of degree $t>2$. Let $\mathbb{E}$ be a central simple alternative algebra of degree two over a nonmodular field $\mathfrak{F}$. Then $\mathfrak{E}$ has order $1,2,4$, or 8 over $\mathfrak{F}$ and has an involutorial antiautomorphism $x \rightarrow \bar{x}$ which is the identity automorphism only when $s=1$. Define $\mathfrak{E}_{t}$ to be the set of all $t$-rowed square matrices $A=\left(a_{i j}\right)$ with $a_{i j}$ in $\mathfrak{S}$ and define $\Im_{t}(\mathfrak{E})$ to be the subspace of $\mathfrak{S}_{t}$ consisting of all matrices $A=\left(a_{i j}\right)$ such that $a_{i j}=\bar{a}_{j i}$. Then $\Im_{\iota}(\mathfrak{S})$ is a subalgebra of $\mathcal{\zeta}_{t}^{(+)}$and is a Jordan algebra except when $t>3$ and $s=8$. Conversely every central simple reduced Jordan algebra is an algebra $\Im_{t}(\mathfrak{S})$.

We now let $\mathfrak{A}$ be a flexible algebra over $\mathfrak{F}$ and assume that $\mathfrak{A}^{(+)}$is a central simple Jordan algebra of degree $t>2$ over $\mathfrak{F}$. Let us assume that $\mathfrak{F}$ has been extended, if necessary, so that $\mathfrak{U}^{(+)}$is a split algebra. We propose to determine the resulting multiplication table of $\mathfrak{A}$. We first write the unity quantity $e$ of $\mathfrak{A}^{(+)}$as a sum $e=e_{1}+\cdots+e_{t}$ of pairwise orthogonal idempotents $e_{i}$ which may be taken to be absolutely primitive. Then $\mathfrak{A}_{e_{i}}(1)=\mathfrak{A}_{i i}$ $=e_{i} \mathfrak{F}$. Decompose $\mathfrak{A}^{(+)}$and so write $\mathfrak{A}$ as the supplementary sum $\mathfrak{A}=\sum_{i<j} \mathfrak{A}_{i j}$ of subspaces $\mathfrak{A}_{i j}$ where $\mathfrak{A}_{i i}$ has already been defined and $\mathfrak{A}_{i j}$ is the intersection of $\mathfrak{A}_{e_{i}}(1 / 2)$ and $\mathfrak{A}_{\Theta_{j}}(1 / 2)$ for $i<j$. The general element $x_{i j}$ of $\mathfrak{A}_{i j}$ may then be regarded as a $t$-rowed square matrix with the element $x$ of an algebra $\sqrt{C}$ in the $i$ th row and $j$ th column, with $\bar{x}$ in the $j$ th row and $i$ th column and with zeros elsewhere. Use the notation $x \cdot y$ to designate products in $\mathbb{E}$ and translate the product in $\mathfrak{A}^{(+)}$in terms of the product in $\mathfrak{A}$ to write

$$
\begin{aligned}
x_{i j} y_{j k}+y_{j k} x_{i j} & =(x \cdot y)_{i k} & & (i<j<k), \\
x_{i j} y_{i k}+y_{i k} x_{i j} & =(\bar{x} \cdot y)_{j k} & & (i<j<k), \\
x_{i k} y_{j k}+y_{j k} x_{i k} & =(x \cdot \bar{y})_{i k} & & (i<j<k) .
\end{aligned}
$$

We also have the results

$$
e_{i} x_{i j}+x_{i j} e_{i}=e_{j} x_{i j}+x_{i j} e_{j}=x_{i j}
$$

Then $\left(e_{i}-e_{j}\right) x_{i j}=x_{i j}\left(e_{j}-e_{i}\right)$ and $\left(e_{i}+e_{j}\right) x_{i j}=x_{i j}\left(e_{i}+e_{j}\right)=x_{i j}$ whence

$$
e_{i} x_{i j}=x_{i j} e_{j}, \quad e_{j} x_{i j}=x_{i j} e_{i}
$$

The subset $\mathfrak{S}_{i j}=\mathfrak{A}_{i i}+\mathfrak{A}_{j j}+\mathfrak{A}_{i j}=\mathfrak{A}_{e_{i+e_{j}}}(1)$ by the known properties of $\mathfrak{A}^{(+)}$ and so $\mathfrak{S}_{i j}$ is a subalgebra of $\mathfrak{A}$. Then $\mathfrak{S}_{i j}^{(+)}$is a central simple Jordan algebra of degree 2 over $\mathfrak{F}, \mathfrak{S}_{i j}$ is stable, 


$$
\mathfrak{A}_{i i} \mathfrak{A}_{i j} \leqq \mathfrak{A}_{i j}, \quad \mathfrak{A}_{i j} \mathfrak{A}_{i i} \leqq \mathfrak{A}_{i j}, \quad \mathfrak{A}_{j j} \mathfrak{A}_{i j} \leqq \mathfrak{A}_{i j}, \quad \mathfrak{A}_{i j} \mathfrak{A}_{j i} \leqq \mathfrak{A}_{i j}
$$

and

$$
\mathfrak{A}_{i i} \mathfrak{A}_{j i}=\mathfrak{A}_{j j} \mathfrak{A}_{i i}=0, \quad \mathfrak{A}_{i j}^{2} \leqq \mathfrak{A}_{i i}+\mathfrak{A}_{j j}+\mathfrak{A}_{i j} .
$$

It is evident from an examination of algebras of degree two that the property $\mathfrak{A}_{i j}^{2} \leqq \mathfrak{A}_{i i}+\mathfrak{A}_{j j}$ is not true in general.

It remains to compute products $x_{i j} y_{k p}$ where at least three of $i, j, k, p$ are distinct. We note first that $\mathfrak{A}_{i j} \mathfrak{A}_{k p}=0$ if neither $i$ nor $j$ is equal to $k$ or $p$. Indeed $\mathfrak{A}_{i j} \leqq \mathfrak{A}_{e_{i+e_{j}}}(1)$ while $\mathfrak{A}_{k p} \leqq \mathfrak{A}_{e_{i+e_{j}}}(0)$. It then remains to compute the products appearing in (27), (28), (29), (30). We observe first that

$$
\mathfrak{A}_{i j} \mathfrak{A}_{j k} \leqq \mathfrak{A}_{i k}, \quad \mathfrak{A}_{j k} \mathfrak{A}_{i j} \leqq \mathfrak{A}_{i k} \quad(i<j<k) .
$$

Indeed $\mathfrak{A}_{i j} \leqq \mathfrak{A}_{e_{i+e_{j}}}(1), \mathfrak{A}_{j k} \leqq \mathfrak{A}_{e_{i+e_{j}}}(1 / 2)$ and so $\mathfrak{A}_{i j} \mathfrak{A}_{j k}$ and $\mathfrak{A}_{j k} \mathfrak{A}_{i j}$ are both subspaces of $\mathfrak{A}_{e_{i}+e_{j}}(1 / 2)$. However they are subsets of the algebra $\mathfrak{A}_{e_{i}+\epsilon_{j+\ell_{k}}}(1)$ $=\mathfrak{A}_{i i}+\mathfrak{A}_{j j}+\mathfrak{A}_{k k}+\mathfrak{A}_{i j}+\mathfrak{A}_{i k}+\mathfrak{A}_{j k}$ and it follows that the products are contained in $\mathfrak{A}_{i k}+\mathfrak{A}_{j k}$. Similarly $\mathfrak{A}_{i j} \leqq \mathfrak{A}_{e_{i}+\ell_{k}}(1 / 2), \mathfrak{A}_{j k} \leqq \mathfrak{A}_{\ell_{j+\ell_{k}}}(1)$ so that their products are subspaces of $\mathfrak{A}_{i k}+\mathfrak{A}_{i j}$. The intersection of these subspaces is $\mathfrak{A}_{i k}$ and we have (26).

We now use the flexibility property $z(x y)+y(x z)=(z x) y+(y x) z$. We take $z=e_{k}$ and compute $e_{k}\left(x_{i j} y_{j k}\right)+y_{j k}\left(x_{i j} e_{k}\right)=\left(e_{k} x_{i j}\right) y_{j k}+\left(y_{j k} x_{i j}\right) e_{k}$. But $x_{i j} e_{k}=e_{k} x_{i j}$ $=0$ and so $e_{k}\left(x_{i j} y_{j k}\right)+\left(x_{i j} y_{j k}\right) e_{k}=\left(x_{i j} y_{j k}+y_{j k} x_{i j}\right) e_{k}$. Since $x_{i j} y_{j k}$ is in $\mathfrak{A}_{i k}$ we have $e_{k}\left(x_{i j} y_{j k}\right)+\left(x_{i j} y_{j k}\right) e_{k}=x_{i j} y_{j k}$. We may then use (23) and have

$$
x_{i j} y_{j k}=e_{i}(x \cdot y)_{i k}=(x \cdot y)_{i k} e_{k}
$$

Since $\left(e_{i}+e_{k}\right)(x \cdot y)_{i k}=(x \cdot y)_{i k}$ we evidently have

$$
y_{j k} x_{i j}=e_{k}(x \cdot y)_{i k}=(x \cdot y)_{i k} e_{i} \quad(i<j<k) .
$$

In exactly the same fashion we compute $e_{k}\left(x_{i j} y_{i k}\right)+y_{i k}\left(x_{i j} e_{k}\right)=\left(e_{k} x_{i j}\right) y_{i k}$ $+\left(y_{i k} x_{i j}\right) e_{k}$ to obtain $x_{i j} y_{i k}=\left(x_{i j} y_{i k}+y_{i k} x_{i j}\right) e_{k}$, that is,

$$
\begin{aligned}
x_{i j} y_{i k} & =e_{i}(\bar{x} \cdot y)_{i k}=(\bar{x} \cdot y)_{i k} e_{k}, \\
y_{i k} x_{i j} & =e_{k}(\bar{x} \cdot y)_{i k}=(\bar{x} \cdot y)_{i k} e_{i} .
\end{aligned} \quad(i<j<k) .
$$

Finally we compute $e_{j}\left(x_{i k} y_{j k}\right)+y_{j k}\left(x_{i k} e_{j}\right)=\left(e_{j} x_{i k}\right) y_{j k}+\left(y_{j k} x_{i k}\right) e_{j}$ whence $x_{i k} y_{j k}$ $=\left(x_{i k} y_{j k}+y_{j k} x_{i k}\right) e_{j}$ and so

$$
\begin{aligned}
& x_{i k} y_{j k}=e_{i}(x \cdot \bar{y})_{i j}=(x \cdot \bar{y})_{i j} e_{j}, \\
& y_{j k} x_{i k}=e_{j}(x \cdot \bar{y})_{i j}=(x \cdot \bar{y})_{i j} e_{i} .
\end{aligned} \quad(i<j<k) .
$$

We have now computed all products required, but have not exhausted the possible implications of the flexible law. Use $z=e_{j}$ and have $e_{j}\left(x_{i j} y_{j k}\right)$ $+y_{j k}\left(x_{i j} e_{j}\right)=\left(e_{j} x_{i j}\right) y_{j k}+\left(y_{j k} x_{i j}\right) e_{j}$. Then $e_{j}\left(x_{i j} y_{j k}\right)=\left(y_{j k} x_{i j}\right) e_{j}=0$ since both $x_{i j} y_{j k}$ and $y_{j k} x_{i j}$ are in $\mathfrak{A}_{i k} \leqq \mathfrak{A}_{e_{j}}(0)$. Then $y_{j k}\left(x_{i j} e_{j}\right)=\left(e_{j} x_{i j}\right) y_{j k}, x_{i j} y_{j k}=\left(e_{i} x_{i j}\right.$ $\left.+x_{i j} e_{j}\right) y_{j k}=\left(x_{i j} e_{j}\right) y_{j k}+y_{j k}\left(x_{i j} e_{j}\right)$. Define 


$$
e_{i} x_{i j}=\left(x S_{i j}\right)_{i j}
$$

where $S_{i j}$ is then a uniquely determined linear transformation of the vector space $(5$. Then we have proved that

$$
x_{i j} y_{j k}=\left(x S_{i j} \cdot y\right)_{i k} \quad(i<j<k) .
$$

To obtain a similar formula with $y$, the transformed vector, we compute $e_{j}\left(y_{j k} x_{i j}\right)+x_{i j}\left(y_{j k} e_{j}\right)=\left(e_{j} y_{j k}\right) x_{i j}+\left(x_{i j} y_{j k}\right) e_{j}$. As before $e_{j}\left(y_{j k} x_{i j}\right)=\left(x_{i j} y_{j k}\right) e_{j}=0$ and so $x_{i j}\left(y_{j k} e_{j}+e_{j} y_{j k}\right)=x_{i j} y_{j k}=\left(e_{j} y_{j k}\right) x_{i j}+x_{i j}\left(e_{j} y_{j k}\right)$, that is,

$$
x_{i j} y_{j k}=\left(x \cdot y S_{j k}\right)_{i k}
$$$$
(i<j<k) \text {. }
$$

However our first value of $x_{i j} y_{j k}$ may be written as $\left[(x \cdot y) S_{i k}\right]_{i k}$ and so we have proved that

$$
x S_{i j} \cdot y=x \cdot y S_{j k}=(x \cdot y) S_{i k} \quad(i<j<k) .
$$

We now take $y=f$ to be the unity quantity of $\mathbb{S}$ and so have $x S_{i j}=x S_{i k}$ for every $x$, that is, $S_{i j}=S_{i k}$ for $i<j<k$. Then $S_{12}=S_{13}=\cdots=S_{1 t}$. Similarly take $x=f$ and have $y S_{j k}=y S_{i k}, S_{i k}=S_{j k}, S_{1 k}=S_{j k}$ for $k>j>1$ and so $S_{12}=S_{13}$ $=S_{23}, S_{j k}=S_{12}$ for all $j<k$ and we have proved that every $S_{i j}=S=S_{12}$. Moreover we now have $x S \cdot y=x \cdot y S=(x \cdot y) S$, that is,

$$
S R_{y}=R_{y} S=R_{y S}
$$

for every $y$ in the algebra $\mathbb{E}$. This implies that $S=R_{f S}$ is commutative with every element of $\mathfrak{S}$ and this is possible for algebras $\mathbb{S}$ of order four and eight, cases where $\mathbb{C}$ is central simple, only when $S$ is a scalar transformation.

Let us now consider the algebra $\mathfrak{S}=\mathfrak{A}_{11}+\mathfrak{A}_{12}+\mathfrak{A}_{22}$, a flexible J-simple subalgebra of $\mathfrak{A}$. The order of $\mathbb{B S}$ is $g=3,4,6$ or 8 according as the order of $\mathbb{E}$ is $1,2,4$ or 8 . We may assume that we are working over an extension of the base field so that (\$) is a split algebra, that is, (\$) has a basis $e=u_{1}, u_{2}, \cdots, u_{g}$ with $u_{i}^{2}=e$. Moreover we may define $e_{1}^{(i)}=\left(e-u_{i}\right) / 2, e_{2}^{(i)}=\left(e+u_{i}\right) / 2$ and then see that the subspace $\mathfrak{A}_{12}$ of $\leftrightarrow$ is spanned by the $g-2$ elements $u_{j}$ for $j \neq 1, i$. When $g=6,8$ the mapping $S=S(i)$ is a scalar mapping for every selection of $e_{1}^{(i)}$ and so $e_{1}^{(i)} u_{j}=\lambda_{i j} u_{j}$. But then $u_{i} u_{j}=\left(e-2 e_{1}^{(i)}\right) u_{j}=\left(1-2 \lambda_{i j}\right) u_{j}$ which is possible, by (18), only if $1-2 \lambda_{i j}=0$, that is, $(5)$ is a Jordan algebra. We have also proved that $\$$ is a Jordan algebra when $g=3$ in $\$ 5.5$.

The multiplication table of $\mathfrak{A}$ has been shown to depend upon relations which can vary only as the algebra $(\$)$ varies. If $\mathfrak{A}$ is taken to be a central simple (split) Jordan algebra we know that $\mathfrak{A}=\mathfrak{A}^{(+)}$and that $\mathfrak{G}$ is a Jordan algebra. But then our study shows that when $\mathbb{S}$ is a Jordan algebra so is $\mathfrak{A}$. If $\mathfrak{B}$ is a total matric algebra the algebra $\mathfrak{B}^{(+)}$is a split Jordan algebra, $\mathfrak{B}$ has the subalgebra $\mathfrak{B}_{0}=\mathfrak{B}_{11}+\mathfrak{B}_{12}+\mathfrak{B}_{22}, \mathfrak{A}=\mathfrak{B}(\lambda)$ has the subalgebra $\mathfrak{B}=\mathfrak{H}_{0}(\lambda)$ $=\mathfrak{A}_{11}+\mathfrak{A}_{12}+\mathfrak{A}_{22}$. Then when $\mathfrak{B}$ is prescribed to be quasiassociative of degree two and order four the structure of $\mathfrak{A}$ is completely known and $\mathfrak{A}=\mathfrak{B}(\lambda)$ is quasiassociative. The extension of the base field which splits $\mathbb{B}$ may now be 
eliminated since clearly a central $\mathrm{J}$-simple algebra $\mathfrak{A}$ is quasiassociative if and only if any scalar extension of $\mathfrak{A}$ is quasiassociative. We have proved

Theorem 13. Let $\mathfrak{A}$ be a flexible J-simple algebra. Then $\mathfrak{A}$ is either a Jordan algebra or is quasiassociative.

We shall now apply our results to a determination of all simple standard algebras. Every simple standard algebra $\mathfrak{A}$ is flexible and $\mathrm{J}$-simple and so is a quasiassociative algebra. Moreover, after a suitable extension of the center of $\mathfrak{A}$, if necessary, we may assume that $\mathfrak{A}=\mathfrak{B}(\lambda)$ where $\mathfrak{B}$ is a total matric algebra. Then $\mathfrak{A}$ has a subalgebra $\mathfrak{H}=\mathfrak{A}_{11}+\mathfrak{A}_{12}+\mathfrak{A}_{22}=\mathfrak{M}_{2}(\lambda)$ where $\mathfrak{M}_{2}$ is the total matric algebra of degree two over $\mathfrak{F}$. It follows that $\mathbb{B}$ has a basis $e, u, v, w$ over $\mathfrak{F}$ where $e$ is the unity quantity of $\&$ and $u^{2}=v^{2}=w^{2}=e, u v=\rho w$, $u w=-\rho v, v w=\rho u$. Since (B) is a standard algebra it satisfies the identity $w[x(y z)]+[(w y) x] z+[(w z) x] y=(w x)(y z)+(w y)(x z)+(w z)(x y)$. Take $y=z$ $=u$ and $x=v$ to obtain $w[v(u u)]+2[(w u) v] u=(w v)(u u)+2(w u)(v u)$. But $w[v(u u)]=w v=(w v)(u u)$ and so $[(w u) v] u=(w u)(v u),[\rho(v v)] u=(\rho v)(-\rho w)$, $\left(\rho+\rho^{3}\right) u=0, \rho\left(1+\rho^{2}\right)=0$. If $\rho=0$ then $\mathscr{H}$ is a Jordan algebra, $\lambda=1 / 2, \mathfrak{A}$ is a Jordan algebra. If $1+\rho^{2}=0$ then $(u v)^{2}=-e$ and $\mathbb{H}$ is associative, $\lambda=1$ and $\mathfrak{A}$ is associative. We have proved

TheOREM 14. A standard simple algebra is either an associative algebra or a Jordan algebra.

8. Problems. We shall close our discussion with a list of some unsolved problems of our theory. A major question is that of the existence of simple nilalgebras in the class of algebras we have called static algebras. An investigation of shrinkable non-commutative algebras of low level seems desirable and might lead to some interesting new types of algebras. A further study of commutative simple algebras also seems desirable. Indeed examples of simple power-associative algebras are needed and some may be furnished by a study which the author will make of the power-associativity of crossed products and some types of crossed extensions. It is desirable to investigate the structure of algebras like Lie algebras and, in particular, of all algebras $\mathfrak{A}$ which are flexible, power-associative, and such that $\mathfrak{H}^{(-)}$is a semi-simple Lie algebra. The type of study which led to our definition of standard algebras may evidently be extended and other forms of Jordan-like identities may yield new classes of simple algebras. Finally, it would be desirable to extend the theory of standard algebras to rings with chain conditions, an extension not yet made even for Jordan algebras.

The University of Chicago, Chicago, Ill. 